\title{
A distribuição Kumaraswamy normal: propriedades, modelos de regressão linear e diagnóstico
}

Elizabete Cardoso Machado

\author{
TESE APRESENTADA \\ $\mathrm{AO}$ \\ Instituto DE MATEMÁticA E EstatísticA \\ Universidade DE SÃo PAUlo \\ PARA \\ OBTENÇAO DO TÍTULO \\ $\mathrm{DE}$ \\ DOUTOR EM CIÊNCIAS
}

Programa: Estatística

Orientadora: Profa. Dra. Denise Aparecida Botter

Coorientadora: Profa. Dra. Mônica Carneiro Sandoval

Durante parte do desenvolvimento deste trabalho a autora recebeu auxílio financeiro da CAPES

São Paulo, Maio de 2019 


\section{A distribuição Kumaraswamy normal: propriedades, modelos de regressão linear e diagnóstico}

Esta versão definitiva da tese contém as correções e alterações sugeridas pela comissão julgadora durante a defesa realizada por Elizabete Cardoso Machado no dia 28/05/2019

Comissão Julgadora:

- Denise Aparecida Botter (Orientadora) - IME-USP

- Gilberto Alvarenga Paula - IME-USP

- Mário de Castro Andrade Filho- ICMC-USP

- Artur José Lemonte - UFRN

- Filidor Edilfonso Vilca Labra- UNICAMP 


\section{Dedicatória}

As mulheres que são capazes de mudar o mundo não precisam mostrar nada além da sua inteligência.

Rita Levi Montalcini 


\section{Agradecimentos}

A Deus, pelo dom da vida, por me guiar durante essa longa caminhada e por sempre me dar força para continuar diante de tantos obstáculos pelos quais tive que passar.

Aos meus pais, meus guerreiros, Maria de Fátima e Cesário pelas lições de vida, pelo incentivo, e principalmente por terem me transmitido os verdadeiros valores da vida: humildade, caráter, honestidade e respeito ao próximo.

Ao meu noivo Bruno Guerra pelo imensurável amor, pela compreensão, pela paciência, pelas palavras de incentivo nas horas mais difíceis, suas palavras foram muito importantes para a concretização deste sonho.

As minhas irmãs: Valderina, Erisvalda e Francisca das Chagas por todo amor, incentivo e carinho.

As minhas amadas sobrinhas, Jennifer (pelas chamadas de vídeos que me motivavam a voltar logo para casa) e Sther pelo carinho incondicional.

A toda a minha família, em especial, meus cunhados Paulo e Marcos pela amizade e torcida.

A Oldênia Guerra e família pela torcida, incentivo e carinho.

As pessoas queridas que estiveram envolvidas na minha formação, meus eternos professores. Agradeço a grande contribuição que deram para a minha vida, pois o conhecimento é para a vida toda. Deixo aqui minha imensa gratidão e carinho.

A minha orientadora Denise Aparecida Botter e coorientadora Mônica Carneiro Sandoval por terem aceitado me orientar em um momento de transição em minha vida. Não poderia ter tido melhor orientação. Obrigada pela confiança em mim depositada e pelos conhecimentos compartilhados

Ao professor Dr. Gauss Montinho Cordeiro pela enorme contribuição neste trabalho de tese.

A Terezinha Késsia e ao professor Dr. Joelson Campos (UFCG) pela enorme ajuda no R, sem a ajuda de vocês as coisas teriam sido bem mais difícil.

Aos demais amigos, que sempre me apoiaram.

Aos meus amigos do doutorado, em especial, Antonio Marcos, Daniel Dataka, Hérica, Agatha, Andressa cerqueira e Guaraci.

Aos professores que aceitaram participar da banca pelas sugestões que irão aprimorar cada vez mais este trabalho.

Aos professores do IME-USP por suas contribuições à minha formação.

Aos professores e amigos do departamento de Estatística da UFPI pelo apoio e torcida.

À CAPES pela concessão de bolsa durante um ano.

A todos os envolvidos diretamente ou indiretamente na construção deste trabalho. 


\section{Resumo}

No presente trabalho, são estudadas propriedades de uma distribuição pertencente à classe de distribuições Kumaraswamy generalizadas, denominada Kumaraswamy normal, formulada a partir da distribuição Kumaraswamy e da distribuição normal. Algumas propriedades estudadas são: expansão da função densidade de probabilidade em série de potências, função geradora de momentos, momentos, função quantílica, entropia de Shannon e de Rényi e estatísticas de ordem. São construídos dois modelos de regressão lineares do tipo localização-escala para a distribuição Kumaraswamy normal, um para dados sem censura e o outro com a presença de observações censuradas. Os parâmetros dos modelos são estimados pelo método de máxima verossimilhança e algumas medidas de diagnóstico, como influência global, influência local e resíduos são desenvolvidos. Para cada modelo de regressão é realizada uma aplicação a um conjunto de dados reais.

Palavras-chave: Função geradora; Distribuição Kumaraswamy normal; classe Lehmann; Desvio medio; Função quantílica. 


\section{Abstract}

In this work, properties of a distribution belonging to the class of generalized Kumaraswamy distributions, called Kumaraswamy normal, are studied. The Kumaraswamy normal distribution is formulated from the Kumaraswamy distribution and from the normal distribution. Some properties studied are: expansion of the probability density function in power series, moment generating function, moments, quantile function, Shannon and Rényi entropy, and order statistics. Two location-scale linear regression models are constructed for the Kumaraswamy normal distribution, one for datas uncensored and the other with the presence of censoreds observations. The parameters of these models are estimated by the maximum likelihood method and some diagnostic measures such as global influence, local influence and residuals are developed. For each regression model an application is made to a real data set.

Keywords: Generating function; Kumaraswamy normal distribution; Lehmann classes; Mean deviation; Moment; Quantile function. 


\section{Sumário}

Lista de Figuras $\quad$ Xv

$\begin{array}{ll}\text { Lista de Tabelas } & \text { xvii }\end{array}$

1 Introdução $\quad \mathbf{1}$

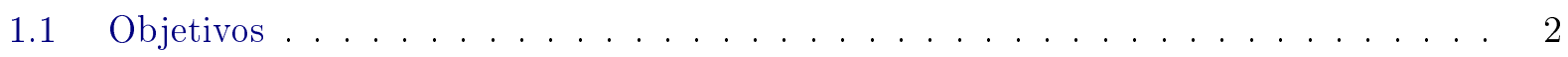

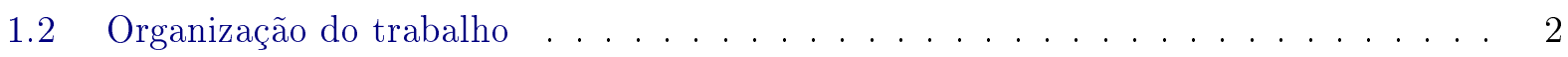

2 A distribuição Kumaraswamy normal $\quad 5$

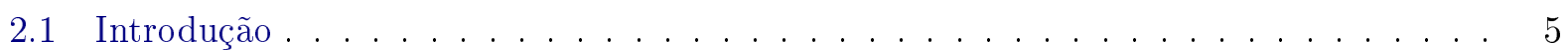

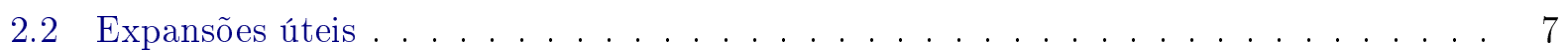

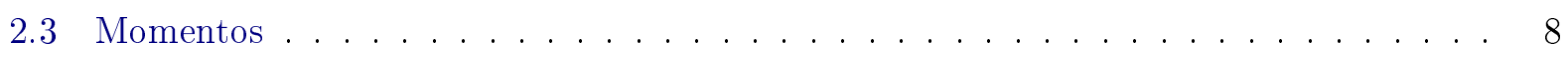

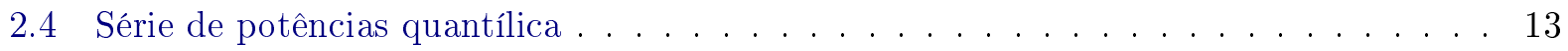

2.5 Funções geradoras . . . . . . . . . . . . . . . . . . . . . . . . . . . . . . . . . . . . . 15

2.6 Momentos incompletos . . . . . . . . . . . . . . . . . . . 17

2.7 Momentos ponderados por probabilidade . . . . . . . . . . . . 18

2.8 Estatísticas de ordem . . . . . . . . . . . . . . . . . . . . . . 19

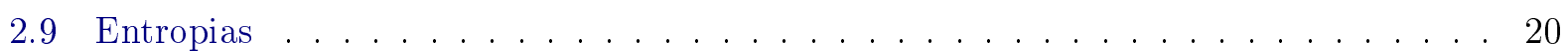

2.10 Estimação . . . . . . . . . . . . . . . . . . . . . . 24

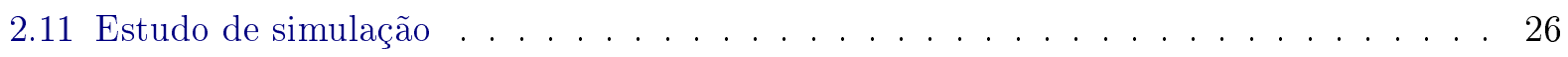

2.12 Aplicação . . . . . . . . . . . . . . . . . . . 26

2.12 .1 Dados de monóxido de carbono . . . . . . . . . . . . . . . 26

3 Modelo de regressão Kumaraswamy normal $\quad 29$

3.1 Introdução . . . . . . . . . . . . . . . . . . . . . . . . . 29

3.2 O modelo de regressão Kumaraswamy normal . . . . . . . . . . . . . . . . . . 29

3.3 Análise de diagnóstico . . . . . . . . . . . . . . . . . . . . . 31

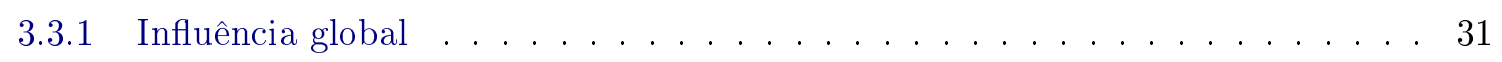

3.3 .2 Influência local . . . . . . . . . . . . . . . . . . . . 31

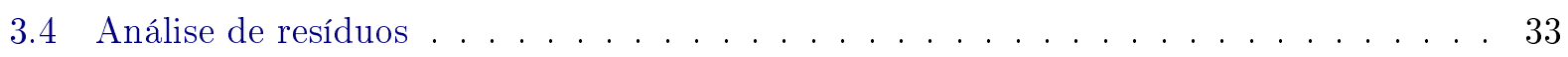

3.5 Aplicação . . . . . . . . . . . . . . . . . . . . 33

3.5.1 Falhas da fotocopiadora . . . . . . . . . . . . 33

4 Modelo de regressão Kumaraswamy normal com presença de censura $\quad 39$

4.1 Introdução . . . . . . . . . . . . . . . . . . . . 39

4.2 Modelo de regressão Kumaraswamy normal com censura . . . . . . . . . . . . . . 39 
4.3 Análise de diagnóstico . . . . . . . . . . . . . . . . . . . . . 41

4.3 .1 Influência local . . . . . . . . . . . . . . . . . . . . . 41

4.4 Análise de resíduos . . . . . . . . . . . . . . . . . . . . . . 42

4.5 Aplicação . . . . . . . . . . . . . . . . . . . 43

4.5.1 Dados de transplante de coração em Stanford . . . . . . . . . . . . . . . . 43

5 Considerações finais $\quad 49$

5.1 Pesquisas futuras . . . . . . . . . . . . . . . . . 49

$\begin{array}{ll}\text { A } & \mathbf{5 1}\end{array}$

A.1 Elementos da matriz de informação observada $J(\theta) \ldots \ldots \ldots \ldots$. . . . . . 51

A.2 Elementos da matriz de informação observada $J(\eta)$ - Modelo de regressão KwN . . . 53

A.3 Elementos da matriz de informação observada $J(\eta)$-Modelo de regressão KwN com

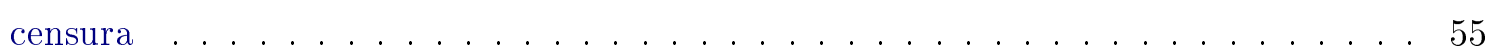

A.4 Dados de transplante de coração em Stanford . . . . . . . . . . . . . . . . 57

$\begin{array}{ll}\text { Referências Bibliográficas } & 61\end{array}$ 


\section{Lista de Figuras}

2.1 Gráfico da função densidade da distribuição $\mathrm{KwN}(\mathrm{a}, \mathrm{b}, 0,1)$ para alguns valores dos parâmetros a e b. . . . . . . . . . . . . . . . . . . . . . 7

2.2 Assimetria de Bowley (a) e curtose de Moors (b) da distribuição KwN para $0<b<3$

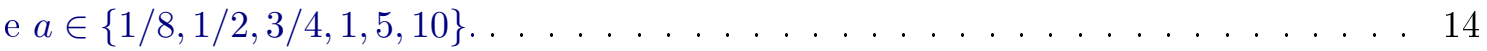

2.3 Assimetria de Bowley (a) e curtose de Moors (b) da distribuição KwN para $0<a<3$

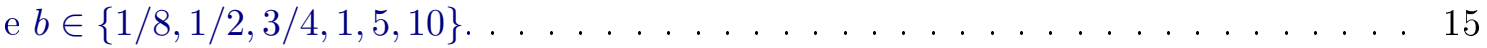

2.4 Gráfico boxplot para os dados de monóxido de carbono. . . . . . . . . . . . . . . . 27

2.5 (a) Densidades estimadas dos modelos KwN e normal para os dados de monóxido de carbono. (b) Funções de distribuição acumuladas estimadas e a fda empírica para os dados de monóxido de carbono. . . . . . . . . . . . . . . . . . . . . . . 28

3.1 Gráfico dos resíduos quantílicos versus valores ajustados para o modelo de regressão KwN. . . . . . . . . . . . . . . . . . . . . . . . 35

3.2 Gráfico de envelope simulado dos resíduos quantílicos para o modelo de regressão KwN. 35

3.3 Gráfico da distância de Cook generalizada para o modelo de regressão KwN. . . . . . 36

3.4 Gráfico de $\left|d_{\max }\right|$ contra índice da observação considerando o esquema de perturbação da ponderação de casos. . . . . . . . . . . . . . . . . . . . . . . . . 36

4.1 Gráficos dos resíduos deviance modificado versus valores ajustados para o modelo de regressão KwN. . . . . . . . . . . . . . . . . . . . . . . . . . . . . . 45

4.2 Gráfico de probabilidade normal com envelope simulado dos resíduos deviance modificado para o modelo de regressão KwN com censura. . . . . . . . . . . . . . . . . 46

4.3 Gráfico de $\left|d_{\max }\right|$ contra índice da observação considerando o esquema de perturbação da ponderação de casos. . . . . . . . . . . . . . . . . . . . . . . 46 


\section{Lista de Tabelas}

2.1 Média e EQM das estimativas dos parâmetros da distribuição KwN de acordo com o tamanho amostral. 26

2.2 Medidas descritivas . . . . . . . . . . . . . . . . . . . . . . . 27

2.3 Estimativas de máxima verossimilhança e respectivos erros padrões dos parâmetros para os modelos de regressão KwN, beta normal (BN), exponenciada normal (Exp-N) e normal ajustados aos dados de CO e valores dos critérios de informação . . . . . . 28

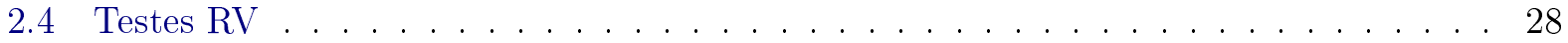

3.1 Estimativas de máxima verossimilhança e respectivos erros padrão dos parâmetros para os modelos de regressão KwN, LTII-N, Exp-N e normal ajustados aos dados do tempo entre falhas da fotocopiadora e valores dos critérios de informação . . . . . . . 35

3.2 Mudanças relativas . . . . . . . . . . . . . . . . . . . . . . . . 36

3.3 Mudanças relativas . . . . . . . . . . . . . . . . . . . . . . . 37

3.4 Estimativas de máxima verossimilhança e respectivos erros padrão dos parâmetros para os modelos de regressão KwN, ajustados aos dados do tempo entre falhas da fotocopiadora sem as observações. . . . . . . . . . . . . . . . . . . . . . . 37

4.1 Estimativas de máxima verossimilhança e respectivos erros padrão dos parâmetros para os modelos de regressão KwN, LTII-N, Exp-N e normal ajustados aos dados de transplante de coração em Stanford . . . . . . . . . . . . . . . . . . . . . . 45

4.2 Critérios de informação . . . . . . . . . . . . . . . . . . . . . . . . . 45

4.3 Mudanças relativas . . . . . . . . . . . . . . . . . . . . . . . . . . 47

4.4 Estimativas de máxima verossimilhança e respectivos erros padrão dos parâmetros para os modelos de regressão KwN, ajustados aos dados de transplante de coração em Stanford sem as observações \#26 e \#91 . . . . . . . . . . . . . . . . . . . . . . . 47

A.1 Dados de transplante de coração em Stanford . . . . . . . . . . . . . . . . . . . . . 57

A.2 Continuação da Tabela anterior . . . . . . . . . . . . . . . . . . . . . . . . 59

A.3 Continuação da Tabela anterior . . . . . . . . . . . . . . . . . . . . . . . . . . 60 


\section{Capítulo 1}

\section{Introdução}

As classes de distribuições generalizadas têm despertado o interesse de muitos pesquisadores motivados pela busca de novas distribuições que se ajustem melhor a fenômenos reais. A forma generalizada de uma distribuição flexibiliza de forma satisfatória a modelagem de dados que apresentam assimetria (SOUZA et al. , 2016).

Essa nova gama de distribuições dá-se principalmente em virtude da adição de novos parâmetros a distribuições clássicas, tais como a distribuição normal (Eugene et al. , 2002). Tahir e Nadarajah (2015) e Tahir e Cordeiro (2016) apresentaram uma revisão abrangente sobre as principais classes de distribuições generalizadas desenvolvidas até 2016.

Amoroso (1925) foi um dos primeiro autores a obter distribuições generalizadas contínuas, abordando em seu trabalho a distribuição gama generalizada. As classes de distribuições generalizadas mais estudadas nos últimos anos foram as exponencializadas, apresentadas inicialmente por Mudholkar et al. (1995), as obtidas pelo método desenvolvido por Marshall e Olkin (1997), as baseadas na distribuição beta (Eugene et al. , 2002), as estendidas discutidas em Barros (2008) e as baseadas na distribuição Kumaraswamy, desenvolvidas por Cordeiro e de Castro (2011).

As propriedades da família exponencializada têm sido estudadas por diversos pesquisadores. Mudholkar e Srivastava (1993) obtiveram a distribuição Weibull exponencializada, Gupta et al. (1998) desenvolveram a classe geral de distribuições exponenciais, Gupta e Kundu (1999) introduziram a distribuição exponencial generalizada, Nadarajah (2005) definiu a distribuição Pareto exponencializada, Nadarajah e Kotz (2006) introduziram a distribuição beta exponencializada, Nadarajah (2006) estudou a distribuição Gumbel exponencializada e Nadarajah (2011) discutiu as propriedades da distribuição exponencial exponencializada.

Desde 2002, diversas distribuições pertencentes à classe de distribuições beta generalizadas (beta-G) surgiram na literatura como, por exemplo, a distribuição beta logística proposta por Ojo e Olapade (2003), a distribuição beta Gumbel introduzida por Nadarajah e Kotz (2004), a distribuição beta exponencial desenvolvida por Nadarajah e Kotz (2006), a distribuição beta exponencial generalizada exponencializada introduzida por Barreto-Souza et al. (2010), a distribuição beta half-normal generalizada proposta por Pescim et al. (2010), a distribuição beta generalizada estudada por (Eugene et al. , 2002) e a distribuição beta Fréchet definida por (Nadarajah e Kotz , 2004).

Barros (2008) apresentou a função de distribuição acumulada, função de densidade de probabilidade e os momentos de várias distribuições estendidas, porém não fez nenhuma aplicação mostrando a eficiência dessas novas distribuições. As distribuições introduzidas por ele foram: 
exponencial estendida, uniforme estendida, Weibull estendida, Pareto estendida, logística padrão estendida, qui-quadrado estendida, gama estendida, Fréchet estendida e Gumbel estendida.

Com respeito à classe de distribuições Kumaraswamy generalizadas, Cordeiro e de Castro (2011) introduziram as distribuições Kw-gama, Kw-Gumbel, Kw-gaussiana inversa, Kw-normal (KwN) e Kw-Weibull. de Pascoa et al. (2011) apresentaram a distribuição Kumaraswamy-gama generalizada, Santana (2010) propôs as distribuições Kumaraswamy-logística e Kumaraswamy-log-logística e Paranaíba (2012) propôs a distribuição Kumaraswamy-Burr XII.

As distribuições generalizadas também desempenham um papel importante em análise de sobrevivência Uma das razões é que a generalização de uma distribuição conhecida pode permitir ao modelo resultante acomodar formas não monótonas para a função de taxa de risco.

Inicialmente, o objetivo era centrado na obtenção das propriedades dessas novas distribuições e, mais recentemente, os estudiosos do tema passaram a incorporar modelos de regressão a elas. Nos trabalhos de Gupta e Kundu (2001), Nadarajah e Kotz (2004), Nadarajah e Kotz (2006) e Andrade et al. (2015) foram investigadas propriedades tais como expansão para função de densidade e função de distribuição, função quantílica, momentos, momentos incompletos, função geradora de momentos, assimetria, curtose, desvio médio, entropia e estimação. Já os trabalhos de Silva et al. (2010), Hashimoto et al. (2010), De Santana et al. (2012) e Cruz et al. (2016) abordam modelos de regressão.

\section{$1.1 \quad$ Objetivos}

Um dos objetivos deste trabalho é estudar as propriedades de uma distribuição pertencente à classe de distribuições Kumaraswamy generalizadas (Cordeiro e de Castro , 2011), denominada Kumaraswamy normal, formulada a partir da distribuição Kumaraswamy e da distribuição normal. Outro objetivo é construir dois modelos de regressão lineares do tipo localização-escala para a distribuição Kumaraswamy normal, um sem censura e o outro com a presença de censura.

\subsection{Organização do trabalho}

Este trabalho está organizado em cinco capítulos. No Capítulo 2, fazemos um estudo aprofundado da distribuição KwN. Várias propriedades estruturais desta distribuição são derivadas, tais como a expansão da função densidade de probabilidade em série de potências, duas expressões para os momentos, função quantílica, função geradora de momentos, momentos incompletos, dois tipos de entropia, Shannon e Rényi e as estatísticas de ordem. No Capítulo 3, propomos um modelo de regressão linear do tipo localização-escala que denominamos de modelo de regressão Kumaraswamy normal (modelo de regressão $\mathrm{KwN}$ ). Estimamos os parâmetros do modelo pelo método de máxima verossimilhança e fornecemos algumas medidas de diagnóstico, como influência global, influência local e resíduos. No Capítulo 4, definimos um modelo de regressão linear do tipo localização-escala para dados censurados que denominamos de modelo de regressão KwN com censura. Os parâmetros do modelo foram estimados usando o método de máxima verossimilhança. Fornecemos também algumas medidas de diagnóstico, como influência local e resíduos. Finalmente, no Capítulo 5, apresentamos as considerações finais. É importante ressaltar que no final de cada capítulo aplicamos a distribuição, discutida ao longo deste trabalho, a conjuntos de dados reais e comparamos os ajustes 
com os de outros modelos. Os gráficos apresentados nesta tese foram produzidos utilizando-se o ambiente de programação $R$ em sua versão 3.3.0 para o sistema operacional Windows que se encontra disponível gratuitamente no endereço http://www.r-project.org. Para mais detalhes ver Ihaka e Gentleman (1996) e Cribari-Neto e Zarkos (2003). 


\section{Capítulo 2}

\section{A distribuição Kumaraswamy normal}

Neste capitulo, estudamos a distribuição Kumaraswamy normal (Cordeiro e de Castro , 2011), que é obtida considerando a distribuição normal como base na família Kumaraswamy-G e apresentamos algumas propriedades estruturais, tais como a função quantílica, momentos ordinários e incompletos e dois tipos de entropia. Consideramos a estimação dos parâmetros do modelo pelo método de máxima verossimilhança e realizamos um estudo de simulação de Monte Carlo com o objetivo de avaliar esses estimadores. Também ilustramos a utilidade da distribuição por meio de uma aplicação a dados reais. O modelo apresenta melhores ajustes que outros modelos sob a mesma distribuição base e também com relação a outros modelos amplamente utilizados.

\subsection{Introdução}

Na última década foram publicadas novas famílias de distribuições contínuas que podem ser úteis para os estatísticos aplicados, generalizando distribuições existentes, por meio da adição de novos parâmetros obtendo-se modelos mais flexíveis. Elas podem servir como alternativas viáveis para outras distribuições na modelagem de dados reais que surgem em vários campos da ciência, como as ciências biológicas, hidrologia, medicina, meteorologia e engenharia, entre outras.

A adição de novos parâmetros de forma para expandir um modelo em uma família maior, a fim de fornecer distribuições assimétricas ou com caudas mais pesadas, desempenha um papel fundamental na teoria de distribuições. Por exemplo, para uma função de distribuição acumulada (fda) contínua $G(x)$, Cordeiro e de Castro (2011) propuseram a distribuição Kumaraswamy-G (Kw-G) tendo fda $F(x)$ dada por

$$
F(x)=1-\left\{1-G(x)^{a}\right\}^{b}, \quad x \in \mathbb{R},
$$

em que $a>0$ e $b>0$ são dois parâmetros de forma.

Para $b=1$, temos a distribuição Lehmann tipo I (LTI) ou a classe exponenciadas-G (expG) com parâmetro $a$. Para $a=1$, obtém-se a classe de distribuições Lehmann tipo II (LTII) com parâmetro $b$ (Lehmann , 1953). Cada novo modelo $\mathrm{Kw}-\mathrm{G}$ pode ser obtido a partir de uma distribuição $\mathrm{G}$ especificada. A fda $G(x)$ é um caso especial de (2.1) quando $a=b=1$.

A função densidade de probabilidade (fdp) correspondente à (2.1) é

$$
f(x)=\operatorname{abg} g(x) G(x)^{a-1}\left\{1-G(x)^{a}\right\}^{b-1}, \quad x \in \mathbb{R},
$$


em que $g(x)=d G(x) / d x$.

A distribuição Kumaraswamy-normal $(\mathrm{KwN})$, objetivo de estudo de nosso trabalho, é definida pela fda

$$
F(x)=1-\left\{1-\Phi\left(\frac{x-\mu}{\sigma}\right)^{a}\right\}^{b}, \quad x \in \mathbb{R},
$$

em que $\mu \in \mathbb{R}$ é um parâmetro de localização, $\sigma>0$ é um parâmetro de escala, $a>0$ e $b>0$ são parâmetros de forma, e $\Phi(\cdot)$ é a função de distribuição acumulada da distribuição normal padrão.

A partir das equações (2.2) e (2.3), a função de sobrevivência da distribuição KwN é dada por

$$
S(x)=P(X>x)=\left[1-\Phi\left(\frac{x-\mu}{\sigma}\right)^{a}\right]^{b}, \quad x \in \mathbb{R},
$$

e a função de risco é expressa por

$$
\lambda(x)=\frac{f(x)}{S(x)}=\frac{a b \phi\left(\frac{x-\mu}{\sigma}\right) \Phi\left(\frac{x-\mu}{\sigma}\right)^{a-1}}{1-\Phi\left(\frac{x-\mu}{\sigma}\right)^{a}}, \quad x \in \mathbb{R} .
$$

A variável aleatória com fda (2.3) é denotada por $X \sim \operatorname{KwN}(a, b, \mu, \sigma)$. Para $\mu=0$ e $\sigma=1$, temos a distribuição KwN padrão.

Seja $Z$ com distribuição $\operatorname{KwN}(a, b, 0,1)$. Então, $X=\mu+\sigma Z$ tem distribuição $\operatorname{KwN}(a, b, \mu, \sigma)$. Daqui em diante, podemos trabalhar apenas com a variável aleatória $Z$ tendo distribuição $\mathrm{KwN}$ padrão, uma vez que as propriedades matemáticas de $X$ podem ser determinadas imediatamente a partir das de $Z$. A fda de $Z$ segue de (2.3) com $\mu=0$ e $\sigma=1$ como

$$
F(x)=1-\left\{1-\Phi(x)^{a}\right\}^{b}, \quad x \in \mathbb{R} .
$$

Assim, a fdp de $Z$ é dada por

$$
f(x)=a b \phi(x) \Phi(x)^{a-1}\left\{1-\Phi(x)^{a}\right\}^{b-1}, \quad x \in \mathbb{R},
$$

em que $\phi(\cdot)$ é a função densidade da distribuição normal padrão. Um dos principais benefícios da distribuição $\mathrm{KwN}$ é a sua capacidade de adaptação a dados reais assimétricos que podem não ser devidamente ajustados por distribuições existentes. Esta distribuição permite uma maior flexibilidade de suas caudas e pode ser amplamente aplicada em muitas áreas de engenharia e biologia. Os parâmetros $a$ e $b$ governam a assimetria e controlam as caudas mais pesadas/leves como forma de fornecer um modelo mais flexível (Figura 2.1). Além disso, pode ser adicionada entropia para o centro da densidade KwN. Note que $\phi(x)$ é uma função densidade simétrica, mas a densidade $f(x)$ será assimétrica.

A equação (2.5) tem propriedades tratáveis especialmente para simulações, uma vez que a função quantílica (fq) de $Z$ tem uma forma simples, ou seja, $z=Q(u)=Q_{S N}\left(\left[1-(1-u)^{1 / b}\right]^{1 / a}\right)$, em que $Q_{S N}(u)=\Phi^{-1}(u)$ é a fq da distribuição normal padrão. Assim, podemos gerar variáveis KwN padronizadas por $z=Q_{S N}\left(\left[1-(1-U)^{1 / b}\right]^{1 / a}\right)$, em que $U$ é uma variável uniforme no intervalo $(0,1)$.

Propriedade 2.1: Se $X \sim K w N\left(a, b, \mu, \sigma^{2}\right)$, então $Y=c X \sim K w N(a, b, c \mu, c \sigma)$. 

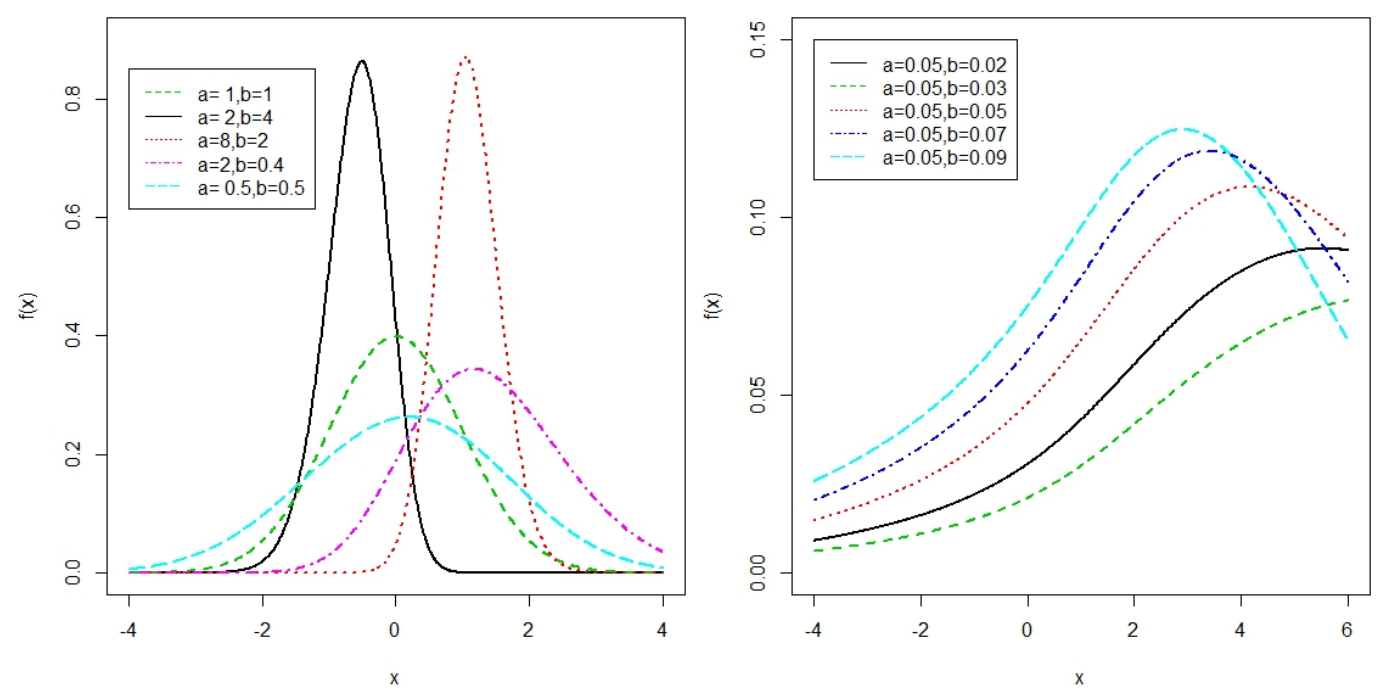

Figura 2.1: Gráfico da função densidade da distribuição $K w N(a, b, 0,1)$ para alguns valores dos parâmetros $a e b$.

Demonstração.

$$
\begin{aligned}
\operatorname{Pr}(Y \leq y) & =\operatorname{Pr}(c X \leq y)=\operatorname{Pr}(X \leq y / c) \\
& =1-\left\{1-\Phi\left(\frac{\frac{y}{c}-\mu}{\sigma}\right)^{a}\right\}^{b} \\
& =1-\left\{1-\Phi\left(\frac{y-c \mu}{c \sigma}\right)^{a}\right\}^{b},
\end{aligned}
$$

ou seja, $c X \sim K w N(a, b, c \mu, c \sigma)$.

\subsection{Expansões úteis}

Nesta seção, obtemos expansões úteis para (2.6) utilizando o conceito de distribuições exponenciadas. A fdp e fda das distribuições exponenciadas normais (com parâmetro $a>0$ ) são dadas por $h_{a}(x)=a \phi(x) \Phi(x)^{a-1}$ e $H_{a}(x)=\Phi(x)^{a}$, respectivamente. As propriedades de várias distribuições exponenciadas têm sido estudadas por alguns autores nos últimos anos (Mudholkar et al. , 1995); (Nadarajah e Gupta, 2007).

Para qualquer número real $d$ e $|z|<1$, a expansão binomial generalizada é definida por

$$
(1-z)^{d}=\sum_{k=0}^{\infty}(-1)^{k}\left(\begin{array}{l}
d \\
k
\end{array}\right) z^{k}
$$

Aplicando a série de potências (2.7) à equação (2.5), a fda de $Z$ pode ser escrita como

$$
F(x)=\sum_{j=0}^{\infty}(-1)^{j+1}\left(\begin{array}{c}
b \\
j+1
\end{array}\right) \Phi(x)^{(j+1) a}=\sum_{j=0}^{\infty}(-1)^{j+1}\left(\begin{array}{c}
b \\
j+1
\end{array}\right) H_{(j+1) a}(x) .
$$


Diferenciando $F(x)$, a fdp de $Z$ em (2.6) pode ser expressa como

$$
\begin{aligned}
f(x)=\sum_{j=0}^{\infty} \frac{(-1)^{j} b\left(\begin{array}{c}
b-1 \\
j
\end{array}\right)}{(j+1)} h_{(j+1) a}(x) & =\sum_{j=0}^{\infty} w_{j} h_{(j+1) a} \\
& =\sum_{j=0}^{\infty} w_{j}(j+1) a \phi(x) \Phi(x)^{(j+1) a-1} \\
& =\phi(x) \sum_{j=0}^{\infty} t_{j} \Phi(x)^{(j+1) a-1},
\end{aligned}
$$

em que $w_{j}=(-1)^{j} b\left(\begin{array}{c}b-1 \\ j\end{array}\right) /(j+1)$ e $t_{j}=(j+1) a w_{j}$.

Em seguida, derivamos uma expansão para $\Phi(x)^{\alpha}$ (para algum $\alpha>0$ real) escrevendo $\Phi(x)^{\alpha}=$ $\{1-[1-\Phi(x)]\}^{\alpha}$ e usando (2.7) duas vezes. Obtemos a série de potências convergente

$$
\begin{aligned}
\Phi(x)^{\alpha} & =\sum_{k=0}^{\infty} \sum_{i=0}^{\infty}(-1)^{k+i}\left(\begin{array}{l}
\alpha \\
i
\end{array}\right)\left(\begin{array}{l}
i \\
k
\end{array}\right) \Phi(x)^{k} \\
& =\sum_{k=0}^{\infty} s_{k}(\alpha) \Phi(x)^{k}
\end{aligned}
$$

em que

$$
s_{k}(\alpha)=\sum_{i=0}^{\infty}(-1)^{k+i}\left(\begin{array}{l}
\alpha \\
i
\end{array}\right)\left(\begin{array}{l}
i \\
k
\end{array}\right) .
$$

Combinando (2.8) e (2.9) e trocando somas, podemos reescrever $f(x)$ como

$$
\begin{aligned}
f(x) & =\phi(x) \sum_{j=0}^{\infty} t_{j} \sum_{k=0}^{\infty} s_{k}[(j+1) a-1] \Phi(x)^{k} \\
& =\phi(x) \sum_{k=0}^{\infty} v_{k} \Phi(x)^{k}
\end{aligned}
$$

em que $v_{k}=\sum_{j=0}^{\infty} t_{j} s_{k}[(j+1) a-1]$.

As equações (2.8) e (2.10) são séries assintóticas convergentes. As quantidades entre parênteses nessas equações funcionam como correções para a função de densidade KwN. Estes são os principais resultados desta seção.

\subsection{Momentos}

Algumas das características interessantes de uma distribuição são aquelas que podem ser estudadas através dos momentos, por exemplo, dispersão, assimetria e curtose. Por isso, precisa-se enfatizar a necessidade e a importância dos momentos em qualquer análise estatística. Nesta seção, derivamos duas representações para o $r$-ésimo momento ordinário da distribuição KwN padrão, definido por $\mu_{r}^{\prime}=E\left(Z^{r}\right)$. Os momentos ordinários de $X \sim \operatorname{KwN}(a, b, \mu, \sigma)$ são imediatamente obtidos a partir dos momentos ordinários de $Z \sim \operatorname{KwN}(a, b, 0,1)$ por $E\left(X^{r}\right)=E\left[(\mu+\sigma Z)^{r}\right]=$ $\sum_{k=0}^{r}\left(\begin{array}{l}r \\ k\end{array}\right) \mu^{r-k} \sigma^{k} E\left(Z^{k}\right)$. 
Definição 2.1. O r-ésimo momento ordinário de uma variável aleatória $Y$ com $f d p f(y)$ é dado por

$$
E\left(Y^{r}\right)=\int_{-\infty}^{\infty} y^{r} f(y) d y
$$

Definição 2.2. O r-ésimo momento central de uma variável aleatória $Y$ com $f d p f(y)$ é dado por

$$
E\left((y-\mu)^{r}\right)=\int_{-\infty}^{\infty}(y-\mu)^{r} f(y) d y
$$

Definição 2.3. A função geradora de cumulantes de uma variável aleatória $Y$ com $f d p f(y)$ é dada por

$$
g(t)=\log [E(\exp (t X))]
$$

O r-ésimo cumulante de $Y$ é o coeficiente $k_{r}$ na expressão de $g(t)$ em série de Taylor dada por $\sum_{r=1}^{\infty} \frac{k_{r} t^{r}}{r !}$.

Teorema 2.1. Para uma variável aleatória $Z \sim K w N(a, b, 0,1)$, o r-ésimo momento ordinário é

$$
\mu_{r}^{\prime}=\sum_{k=0}^{\infty} v_{k} \tau_{r, k},
$$

em que $\tau_{r, k}$ é o $(r, k)$-ésimo momento ponderado por probabilidade (PWM) (para $r$ e $k$ inteiros positivos) da distribuição normal padrão.

Demonstração. De fato, a quantidade $\mu_{r}^{\prime}$ pode ser derivada a partir de (2.10) e da definição em (2.11) como

$$
\begin{aligned}
\mu_{r}^{\prime} & =\int_{-\infty}^{\infty} x^{r} \sum_{k=0}^{\infty} v_{k} \Phi(x)^{k} \phi(x) d x \\
& =\sum_{k=0}^{\infty} v_{k} \int_{-\infty}^{\infty} x^{r} \Phi(x)^{k} \phi(x) d x=\sum_{k=0}^{\infty} v_{k} \tau_{r, k}
\end{aligned}
$$

em que $\tau_{r, k}$ é dado por

$$
\tau_{r, k}=\int_{-\infty}^{\infty} x^{r} \Phi(x)^{k} \phi(x) d x
$$

Agora, fornecemos uma expressão para $\tau_{r, k}$. A fda da distribuição normal padrão pode ser escrita como

$$
\Phi(x)=\frac{1}{2}\left\{1+\operatorname{erf}\left(\frac{\mathrm{x}}{\sqrt{2}}\right)\right\}, \quad x \in \mathbb{R},
$$

em que erf $(x)$ é a série de potências para a função erro definida por

$$
\operatorname{erf}(x)=\frac{2}{\sqrt{\pi}} \sum_{m=0}^{\infty} \frac{(-1)^{m} x^{2 m+1}}{(2 m+1) m !}
$$

Como estamos integrando no intervalo do raio de convergência em torno de 0 , a série de potências para erf $(x)$ também converge para todos os valores reais. Para pequenos valores de $|x|$, particularmente aqueles menores do que 1, a convergência desta série é bastante rápida com um 
pequeno número de termos. No entanto, quando $|x|$ é grande, a convergência pode ser bastante lenta, pelo menos inicialmente.

Usando a expansão binomial dada em (2.7) e trocando termos, obtemos

$$
\begin{aligned}
\tau_{r, k} & =\frac{1}{2^{k} \sqrt{2 \pi}} \sum_{p=0}^{k}\left(\begin{array}{l}
k \\
p
\end{array}\right) \int_{-\infty}^{\infty} x^{r} \operatorname{erf}\left(\frac{x}{\sqrt{2}}\right)^{k-p} \exp \left(-\frac{x^{2}}{2}\right) d x \\
& =\frac{1}{2^{k} \sqrt{2 \pi}} \sum_{p=0}^{k}\left(\begin{array}{l}
k \\
p
\end{array}\right) I(k, p),
\end{aligned}
$$

em que $I(k, p)=\int_{-\infty}^{\infty} x^{r} \operatorname{erf}\left(\frac{x}{\sqrt{2}}\right)^{k-p} \exp \left(-\frac{x^{2}}{2}\right) d x$

Com base na série de potências para erf $(x)$ acima, obtemos $\tau_{r, k}$ a partir de resultados de Nadarajah (2008, Equações (9) - (11)). Primeiro, necessitamos da função Lauricella do tipo A definida por

$$
\begin{aligned}
& F_{A}^{(n)}\left(a ; b_{1}, \ldots, b_{n} ; c_{1}, \ldots, c_{n} ; x_{1}, \ldots, x_{n}\right)= \\
& \sum_{m_{1}=0}^{\infty} \cdots \sum_{m_{n}=0}^{\infty} \frac{(a)_{m_{1}+\cdots+m_{n}}\left(b_{1}\right)_{m_{1}} \cdots\left(b_{n}\right)_{m_{n}}}{\left(c_{1}\right)_{m_{1}} \cdots\left(c_{n}\right)_{m_{n}}} \frac{x_{1}^{m_{1}} \cdots x_{n}^{m_{n}}}{m_{1} ! \cdots m_{n} !}
\end{aligned}
$$

Usando a série de potências para a função erro, temos

$$
\begin{aligned}
I(k, p)= & \int_{-\infty}^{\infty} x^{r} \exp \left(-\frac{x^{2}}{2}\right)\left[\frac{2}{\sqrt{\pi}} \sum_{m=0}^{\infty} \frac{(-1)^{m} x^{2 m+1}}{2^{m+\frac{1}{2}}(2 m+1) m !}\right]^{k-p} d x \\
= & \left(\frac{2}{\sqrt{\pi}}\right)^{k-p} \sum_{m_{1}=0}^{\infty} \ldots \sum_{m_{k-p}=0}^{\infty} \frac{(-1)^{m_{1}+\ldots+m_{k-p}}}{2^{m_{1}+\ldots+m_{k-p}+\frac{k-p}{2}}\left(2 m_{1}+1\right) \ldots\left(2 m_{k-p}+1\right) m_{1} ! \ldots m_{k-p} !} \\
& \times \int_{-\infty}^{\infty} x^{2\left(m_{1}+\ldots+m_{k-p}\right)+r+k-p} \exp \left(-\frac{x^{2}}{2}\right) d x \\
= & \left(\frac{2}{\sqrt{\pi}}\right)^{k-p} \sum_{m_{1}=0}^{\infty} \ldots \sum_{m_{k-p}=0}^{\infty} \frac{(-1)^{m_{1}+\ldots+m_{k-p}}}{2^{m_{1}+\ldots+m_{k-p}+\frac{k-p}{2}}\left(2 m_{1}+1\right) \ldots\left(2 m_{k-p}+1\right) m_{1} ! \ldots m_{k-p} !} \\
& \times 2^{m_{1}+\ldots+m_{k-p}+\frac{r+k-p+1}{2}} \int_{-\infty}^{\infty}\left(\frac{x^{2}}{2}\right)^{\left(m_{1}+\ldots+m_{k-p}+\frac{r+k-p+1}{2}\right)-1} \exp \left(-\frac{x^{2}}{2}\right) d x \\
= & \left(\frac{2}{\sqrt{\pi}}\right)^{k-p} 2^{\frac{r+1}{2}} \sum_{m_{1}=0}^{\infty} \ldots \sum_{m_{k-p}=0}^{\infty} \frac{(-1)^{m_{1}+\ldots+m_{k-p}}}{2^{k-p}\left(m_{1}+\frac{1}{2}\right) \ldots\left(m_{k-p}+\frac{1}{2}\right) m_{1} ! \ldots m_{k-p} !} \\
& \times \Gamma\left(m_{1}+\ldots+m_{k-p}+\frac{r+k-p+1}{2}\right) \\
= & \pi^{\frac{k-p}{2}} 2^{\frac{r+1}{2}} \sum_{m_{1}=0}^{\infty} \ldots \sum_{m_{k-p}=0}^{\infty} \frac{(-1)^{m_{1}+\ldots+m_{k-p}}}{\left(m_{1}+\frac{1}{2}\right) \ldots\left(m_{k-p}+\frac{1}{2}\right) m_{1} ! \ldots m_{k-p} !} \\
& \times \Gamma\left(m_{1}+\ldots+m_{k-p}+\frac{r+k-p+1}{2}\right)
\end{aligned}
$$

para $r+k-p$ par. Agora, usando o fato de que $(f)_{k}=\Gamma(f+k) / \Gamma(f)$ e a definição em (2.14), 
podemos simplificar (2.15) para

$$
\begin{aligned}
I(k, p)= & \pi^{\frac{p-k}{2}} 2^{\frac{r+1}{2}} \Gamma\left(\frac{r+k-p+1}{2}\right) \sum_{m_{1}=0}^{\infty} \ldots \sum_{m_{k-p}=0}^{\infty} \frac{(r+k-p+1)_{m_{1}+\ldots+m_{k-p}}(-1)^{m_{1}+\ldots+m_{k-p}}}{\left(m_{1}+\frac{1}{2}\right) \ldots\left(m_{k-p}+\frac{1}{2}\right) m_{1} ! \ldots m_{k-p} !} \\
= & \pi^{\frac{p-k}{2}} 2^{\frac{r+1}{2}+k-p} \Gamma\left(\frac{r+k-p+1}{2}\right) \\
& \times F_{A}^{k-p}\left(\frac{r+k-p+1}{2} ; \frac{1}{2}, \ldots, \frac{1}{2} ; \frac{3}{2}, \ldots \frac{3}{2} ;-1, \ldots,-1\right) .
\end{aligned}
$$

Combinando (2.13) e (2.16), se $r+k-p$ é par, podemos escrever

$$
\begin{aligned}
\tau_{r, k}= & 2^{r / 2} \pi^{-(k+1 / 2)} \sum_{p=0}^{k}\left(\frac{\pi}{2}\right)^{p}\left(\begin{array}{l}
k \\
p
\end{array}\right) \Gamma\left(\frac{r+k-p+1}{2}\right) \\
& \times F_{A}^{(k-p)}\left(\frac{r+k-p+1}{2} ; \frac{1}{2}, \ldots, \frac{1}{2} ; \frac{3}{2}, \ldots, \frac{3}{2} ;-1, \ldots,-1\right),
\end{aligned}
$$

em que os termos de $\tau_{r, k}$ desaparecem quando $r+k-p$ é ímpar. Então, os momentos de $Z$ podem ser determinados a partir da equação (2.12) e das quantidades $\tau_{r, k}$ em (2.17).

Ao longo deste trabalho, aplicamos a equação de Gradshteyn e Ryzhik (2008, Seção 0.314) para uma série de potências gerada para qualquer inteiro positivo $k$, dada por

$$
\left(\sum_{m=0}^{\infty} a_{m} x^{m}\right)^{k}=\sum_{m=0}^{\infty} c_{k, m} x^{m}
$$

em que $c_{k, 0}=a_{0}^{k}$ e os coeficientes $c_{k, m}$ (para $m=1,2, \ldots$ ) são determinados recursivamente a partir da equação

$$
c_{k, m}=\left(k a_{0}\right)^{-1} \sum_{n=1}^{m}[n(k+1)-m] a_{n} c_{k, m-n} .
$$

A segunda fórmula para $\mu_{r}^{\prime}$ baseia-se na fq da distribuição normal padrão e é descrita no Teorema a seguir.

Teorema 2.2. Se $Z \sim K w N(a, b, 0,1)$, então a partir da fq da distribuição normal padrão o $r$-ésimo momento ordinário de $Z$ pode ser escrito como

$$
\mu_{r}^{\prime}=\sum_{n, l, k=0}^{\infty} \frac{(-1 / 2)^{n-l}(\sqrt{2 \pi})^{n} f_{r, n} v_{k}}{l+k+1}\left(\begin{array}{l}
n \\
l
\end{array}\right) .
$$

Demonstração. Com efeito, segue da equação em (2.10) e da definição em (2.11) que

$$
\mu_{r}^{\prime}=\int_{-\infty}^{\infty} x^{r} \sum_{k=0}^{\infty} v_{k} \Phi(x)^{k} \phi(x) d x .
$$

Sendo $u=\Phi(x)$, segue que $x=\Phi^{-1}(u)$. Dessa forma, trocando a soma e a integral podemos reescrever $\mu_{r}^{\prime}$ acima em termos de $Q_{S N}(u)=\Phi^{-1}(u)$ como

$$
\mu_{r}^{\prime}=\sum_{k=0}^{\infty} v_{k} \int_{0}^{1} Q_{S N}(u)^{r} u^{k} d u
$$


A fq da distribuição normal padrão pode ser expandida em uma série de potências convergente (ver Steinbrecher , 2002)

$$
Q_{S N}(u)=\sum_{i=0}^{\infty} b_{i} v^{2 i+1}
$$

em que $v=\sqrt{2 \pi}(u-1 / 2)$ e os $b_{i}^{\prime} \mathrm{s}$ são determinados recursivamente a partir de

$$
b_{i+1}=\frac{1}{2(2 i+3)} \sum_{r=0}^{i} \frac{(2 r+1)(2 i-2 r+1) b_{r} b_{i-r}}{(r+1)(2 r+1)} .
$$

Aqui, $b_{0}=1, b_{1}=1 / 6, b_{2}=7 / 120, b_{3}=127 / 7560, \ldots$

Podemos reescrever (2.20) como

$$
Q_{S N}(u)=\sum_{n=0}^{\infty} d_{n} v^{n}
$$

em que os coeficientes $d_{n}^{\prime}$ s são dados por $d_{n}=0$ para $i=0,2,4, \ldots$ e $d_{n}=b_{(n-1) / 2}$ para $n=$ $1,3,5, \ldots$

Em seguida, baseado em (2.18) e (2.21), podemos escrever $Q_{S N}(u)^{r}=\left(\sum_{n=0}^{\infty} d_{n} v^{n}\right)^{r}=\sum_{n=0}^{\infty} f_{r_{n}} v^{n}$, em que $f_{r, n}=\left(n a_{0}\right)^{-1} \sum_{i=1}^{n}[i(r+1)-n] d_{i} f_{k r, n-i}($ para $n \geq 0)$ e $f_{0, n}=d_{0}^{n}$.

Segue das equações (2.19) e (2.20) que

$$
\begin{aligned}
\mu_{r}^{\prime} & =\sum_{k=0}^{\infty} v_{k} \int_{0}^{1} \sum_{n=0}^{\infty} f_{r, n} v^{n} u^{k} d u \\
& =\sum_{k=0}^{\infty} \sum_{n=0}^{\infty} f_{r, n} v_{k} \int_{0}^{1} v^{n} u^{k} d u \\
& =\sum_{k=0}^{\infty} \sum_{n=0}^{\infty} f_{r, n} v_{k} \int_{0}^{1}[\sqrt{2 \pi}(u-1 / 2)]^{n} u^{k} d u
\end{aligned}
$$

Usando o resultado $\int_{0}^{1}(u-1 / 2)^{n} u^{k} d u=\sum_{l=0}^{\infty} \frac{(-1 / 2)^{n-l}}{l+k+1}\left(\begin{array}{l}n \\ l\end{array}\right)$, obtemos

$$
\begin{aligned}
\mu_{r}^{\prime} & =\sum_{k=0}^{\infty} \sum_{n=0}^{\infty} \sum_{l=0}^{\infty} \frac{(-1 / 2)^{n-l}(\sqrt{2 \pi})^{n} f_{r, n} v_{k}}{l+k+1}\left(\begin{array}{c}
n \\
l
\end{array}\right) \\
& =\sum_{n, l, k=0}^{\infty} \frac{(-1 / 2)^{n-l}(\sqrt{2 \pi})^{n} f_{r, n} v_{k}}{l+k+1}\left(\begin{array}{c}
n \\
l
\end{array}\right) .
\end{aligned}
$$

Portanto, $\mu_{r}^{\prime}$ é expresso como

$$
\mu_{r}^{\prime}=\sum_{n, l, k=0}^{\infty} \frac{(-1 / 2)^{n-l}(\sqrt{2 \pi})^{n} f_{r, n} v_{k}}{l+k+1}\left(\begin{array}{l}
n \\
l
\end{array}\right) .
$$


As equações (2.12) e (2.22) são os principais resultados desta seção. Elas podem ser calculadas usando plataformas analíticas como MATHLAB e MATHEMATICA com 20 ou 30 termos. As expansões algébricas estabelecidas para determinar os momentos de $Z$ destas equações podem ser mais eficientes do que computá-las pela integração numérica de (2.6), que pode ser propensa a erros de arredondamento, entre outros.

Os momentos centrais $\left(\mu_{s}\right)$ e cumulantes $\left(\kappa_{s}\right)$ de $Z$ são determinados a partir dos momentos ordinários como $\mu_{s}=\sum_{k=0}^{s}\left(\begin{array}{l}s \\ k\end{array}\right)(-1)^{k} \mu_{1}^{s} \mu_{s-k}^{\prime}$ e $\kappa_{s}=\mu_{s}^{\prime}-\sum_{k=1}^{s-1}\left(\begin{array}{c}s-1 \\ k-1\end{array}\right) \kappa_{k} \mu_{s-k}^{\prime}$, respectivamente, em que $\kappa_{1}=\mu_{1}^{\prime}$. Os coeficientes de assimetria $\left(\gamma_{1}=\kappa_{3} / \kappa_{2}^{3 / 2}\right)$ e curtose $\left(\gamma_{2}=\kappa_{4} / \kappa_{2}^{2}\right)$ de $Z$ são apenas o terceiro e quarto cumulantes padronizados. Elas são importantes para derivar expansões de Edgeworth para a fda e fdp da soma padronizada e da média de variáveis aleatórias independentes e identicamente distribuídas seguindo a distribuição KwN padrão.

O $r$-ésimo momento fatorial de $Z$ é $\mu_{(r)}^{\prime}=E\left(Z^{(r)}\right)=E[Z(Z-1) \times \cdots \times(Z-r+1)]=$ $\sum_{l=0}^{r} s(r, l) \mu_{r}^{\prime}$, em que $s(r, l)=(l !)^{-1}\left[d^{l} l^{(r)} / d x^{l}\right]_{x=0}$ é o número de Stirling do primeiro tipo (Abramowitz e Stegun, 1965).

\subsection{Série de potências quantílica}

A função quantílica é útil para obter várias propriedades matemáticas de distribuições. Em alguns casos, basta inverter a fda para obter a função quantílica. No entanto, para outras distribuições, essa solução não é possível. Nesses casos, pode-se adotar métodos de série de potências para a obtenção da função quantílica.

A fq de $Z$ é dada por $\left.z=Q(u)=Q_{S N}\left(\left[1-(1-u)^{1 / b}\right]^{1 / a}\right]\right)$ (ver Seção 2.1). Os efeitos dos parâmetros de forma adicionais $a$ e $b$ na assimetria e curtose de $Z$ podem ser baseados em medidas quantílicas. A assimetria de Bowley (Kenney e Keeping, 1962) é dada por

$$
B=\frac{Q(3 / 4)+Q(1 / 4)-2 Q(1 / 2)}{Q(3 / 4)-Q(1 / 4)}
$$

A curtose de Moors (Moors, 1988) é dada por

$$
M=\frac{Q(3 / 8)-Q(1 / 8)+Q(7 / 8)-Q(5 / 8)}{Q(6 / 8)-Q(2 / 8)} .
$$

Estas medidas são pouco sensíveis a outliers e existem mesmo para distribuições sem momentos. Nas Figuras 2.2 e 2.3 exibimos gráficos destas medidas como funções dos parâmetros $a$ e $b$ da distribuição KwN, respectivamente. Estes gráficos mostram que ambas as medidas são muito sensíveis dependendo dos valores fixados para os parâmetros de forma.

Expandindo o binômio em $v$ na equação (2.21), temos

$$
Q_{S N}(u)=\sum_{i=0}^{\infty} \sum_{m=0}^{i}(2 \pi)^{i / 2}(-1 / 2)^{i-m} d_{i}\left(\begin{array}{c}
i \\
m
\end{array}\right) u^{m} .
$$

Permutando $\sum_{i=0}^{\infty} \sum_{m=0}^{i}$ por $\sum_{m=0}^{\infty} \sum_{i=m}^{\infty}$, obtemos

$$
Q_{S N}(u)=\sum_{m=0}^{\infty} p_{m} u^{m}
$$



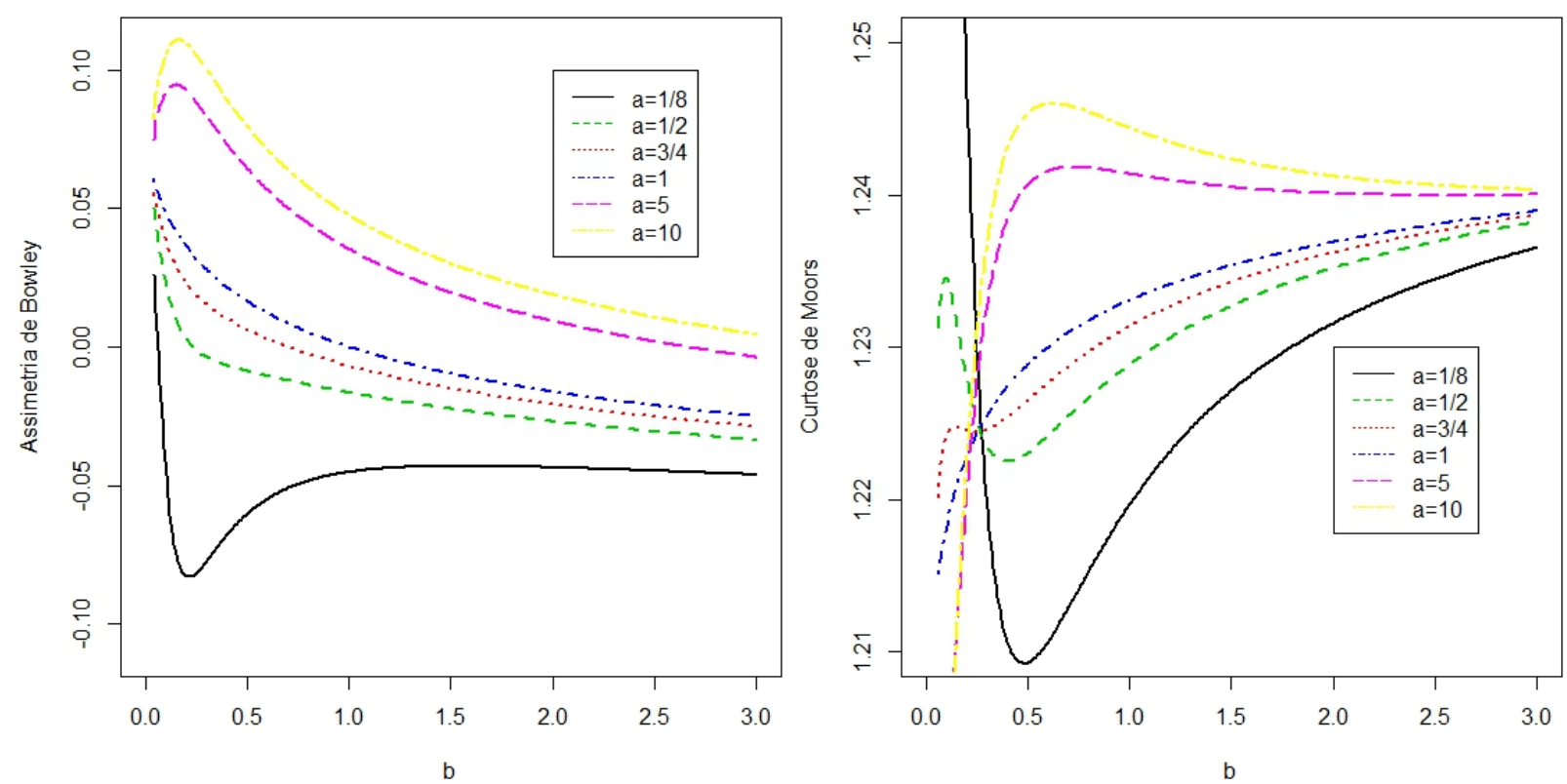

Figura 2.2: Assimetria de Bowley (a) e curtose de Moors (b) da distribuição KwN para $0<b<3$ e $a \in\{1 / 8,1 / 2,3 / 4,1,5,10\}$.

em que $p_{m}=\sum_{i=m}^{\infty}(2 \pi)^{i / 2}(-1 / 2)^{i-m} d_{i}\left(\begin{array}{c}i \\ m\end{array}\right)$.

Com base na última equação, a fq de $Z$ torna-se

$$
Q(u)=\sum_{m=0}^{\infty} p_{m}\left[1-(1-u)^{1 / b}\right]^{m / a} .
$$

Usando (2.7) duas vezes, obtemos

$$
\begin{aligned}
{\left[1-(1-u)^{1 / b}\right]^{m / a} } & =\sum_{k=0}^{\infty}(-1)^{k}\left(\begin{array}{c}
m / a \\
k
\end{array}\right)(1-u)^{k / b} \\
& =\sum_{k=0}^{\infty}(-1)^{k}\left(\begin{array}{c}
m / a \\
k
\end{array}\right) \sum_{j=0}^{\infty}(-1)^{j}\left(\begin{array}{c}
k / b \\
j
\end{array}\right) u^{j} \\
& =\sum_{j=0}^{\infty} \sum_{k=0}^{\infty}(-1)^{k+j}\left(\begin{array}{c}
m / a \\
k
\end{array}\right)\left(\begin{array}{c}
k / b \\
j
\end{array}\right) u^{j}=\sum_{j=0}^{\infty} \nu_{j, m} u^{j}
\end{aligned}
$$

em que $\nu_{j, m}=\sum_{k=0}^{\infty}(-1)^{k+j}\left(\begin{array}{c}m / a \\ k\end{array}\right)\left(\begin{array}{c}k / b \\ j\end{array}\right)$. Então, podemos reescrever $Q(u)$ como

$$
Q(u)=\sum_{j=0}^{\infty} \eta_{j} u^{j}
$$

em que $\eta_{j}=\sum_{m=0}^{\infty} p_{m} \nu_{j, m}$.

A equação (2.23) indica que a função quantílica da distribuição $\mathrm{KwN}$ pode ser expressa como uma série de potências. Assim, várias propriedades matemáticas de $Z$ podem ser dadas em termos de integrais sobre $(0,1)$. De fato, seja $W(\cdot)$ uma função integrável na reta real. Podemos escrever

$$
\int_{-\infty}^{\infty} W(x) f(x) d x=\int_{0}^{1} W\left(\sum_{j=0}^{\infty} \eta_{j} u^{j}\right) d u .
$$



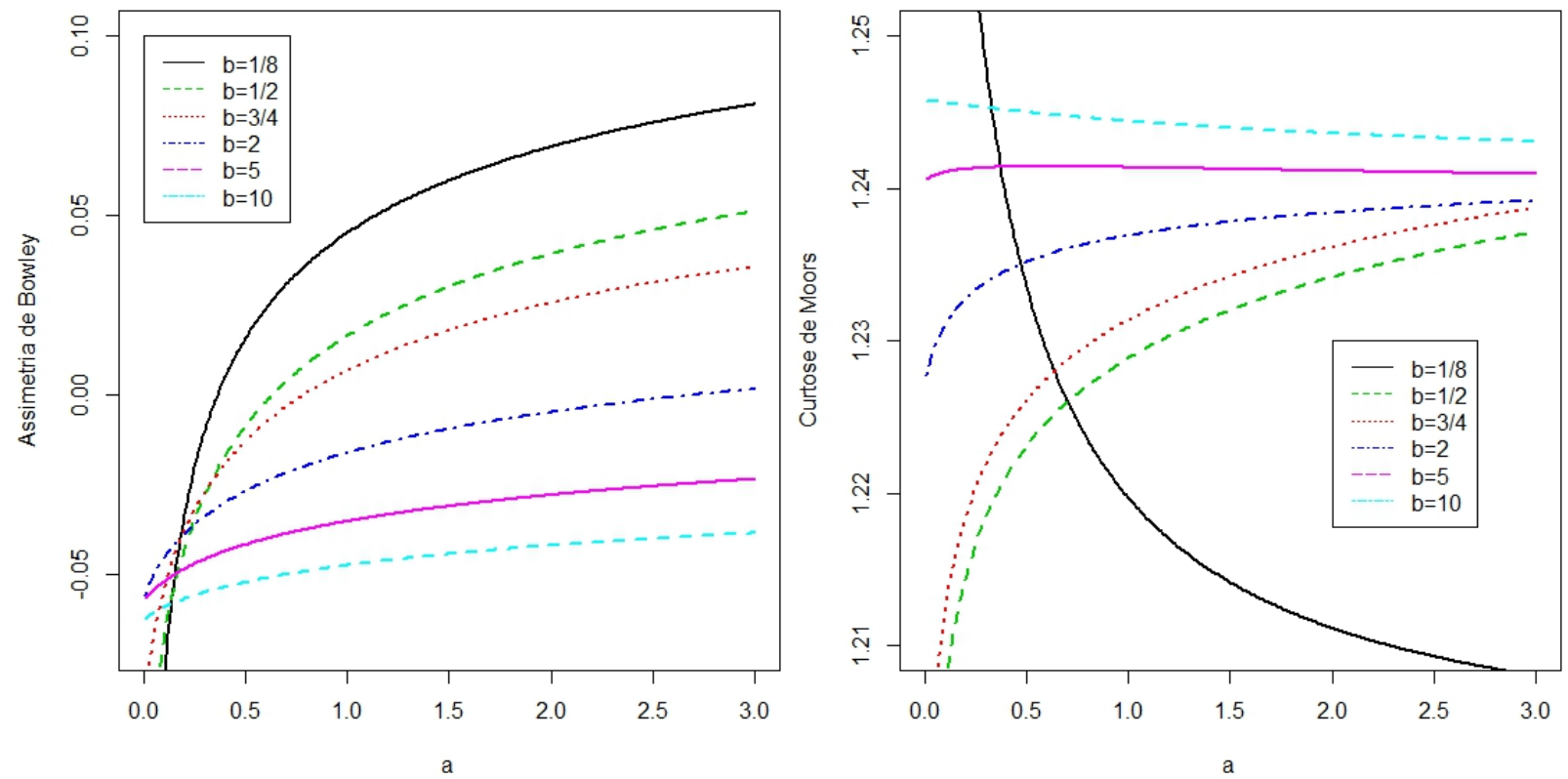

Figura 2.3: Assimetria de Bowley (a) e curtose de Moors (b) da distribuição $K w N$ para $0<a<3 e$ $b \in\{1 / 8,1 / 2,3 / 4,1,5,10\}$.

Com efeito,

$$
\int_{-\infty}^{\infty} W(x) f(x) d x=\int_{-\infty}^{\infty} W(x) d F(x)
$$

Fazendo a mudança de variável $F(x)=u$, temos

$$
\int_{-\infty}^{\infty} W(x) f(x) d x=\int_{-\infty}^{\infty} W(x) d F(x)=\int_{0}^{1} W\left(F^{-1}(u)\right) d u
$$

Lembrando que $F^{-1}(u)=Q(u)$ e substituindo a equação (2.23) na última integral acima, obtemos

$$
\int_{-\infty}^{\infty} W(x) f(x) d x=\int_{0}^{1} W\left(\sum_{j=0}^{\infty} \eta_{j} u^{j}\right) d u .
$$

As equações (2.23) e (2.24) são os principais resultados desta seção. Elas são muito úteis para determinar várias propriedades estruturais da $\mathrm{KwN}$ usando funções especiais $W(\cdot)$. Além disso, a integral do lado direito é geralmente mais simples do que a integral do lado esquerdo. Apresentamos aplicações das expressões (2.23) e (2.24) para obter funções geradoras e momentos incompletos nas duas seções a seguir.

\subsection{Funções geradoras}

A função geradora de momentos (fgm), se existir, determina de forma unívoca a distribuição da variável aleatória, ou seja, existe uma única distribuição com função geradora $M(t)$. Por outro lado, se duas variáveis possuem uma mesma função geradora, então possuem uma mesma distribuição. Aqui, obtemos a fgm da distribuição $\mathrm{KwN}(a, b, 0,1)$. 
Definição 2.4. A função geradora de momentos de uma variável aleatória $Y$ é definida por

$$
M(t)=E[\exp (t Y)], \quad t \in \mathbb{R} .
$$

Teorema 2.3. Se $Z \sim K w N(a, b, 0,1)$, então a fgm de $Z$ é dada por

$$
M(t)=\exp \left(\eta_{0} t\right) \sum_{k=0}^{\infty} s_{k} t^{k}
$$

em que $s_{k}=\sum_{n=0}^{\infty} B_{n, k} /(n+1)$ ! (para $\left.k \geq 0\right)$ e as quantidades $B_{n, k}=B_{n, k}\left(\eta_{1}, 2 \eta_{2}, \ldots,(n-k+\right.$ $\left.1) ! \eta_{n-k+1}\right)$ podem ser facilmente determinadas.

Demonstração. A partir da Definição 2.4 e das equações (2.23) e (2.24), podemos escrever

$$
\begin{aligned}
M(t) & =\int_{-\infty}^{\infty} \exp (t x) f(x) d x \\
& =\int_{0}^{1} \exp \left(t \sum_{j=0}^{\infty} \eta_{j} u^{j}\right) d u \\
& =\exp \left(\eta_{0} t\right) \int_{0}^{1} \exp \left(t \sum_{j=1}^{\infty} \eta_{j} u^{j}\right) d u
\end{aligned}
$$

Os polinômios exponenciais parciais de Bell são definidos por Comtet (1974) como

$$
\exp \left(t \sum_{j=1}^{\infty} \eta_{j} \frac{u^{j}}{j !}\right)=\sum_{n, k=0}^{\infty} \frac{B_{n, k}}{n !} u^{n} t^{k},
$$

em que

$$
B_{n, k}=B_{n, k}\left(\eta_{1}, \eta_{2}, \ldots, \eta_{n-k+1}\right)=\sum \frac{n ! \eta_{1}^{c_{1}} \eta_{2}^{c_{2}} \ldots \eta_{n-k+1}^{c_{n-k+1}}}{c_{1} ! c_{2} ! \ldots c_{n-k+1} !(1 !)^{c_{1}}(2 !)^{c_{2}} \ldots(n-k+1) ! c^{c_{n-k+1}}},
$$

e a soma ocorre sobre todos os inteiros $c_{1}, c_{2}, \ldots \geq 0$ que verificam $c_{1}+2 c_{2}+3 c_{3}+\cdots=n$ e $c_{1}+c_{2}+c_{3}+\cdots=k$. Estes polinômios podem ser computados como BellY $[\mathbf{n}, \mathbf{k},\{\mathbf{1}, \ldots, \mathbf{n}-\mathbf{k}+\mathbf{1}\}]$ no MATHEMATICA e como IncompleteBellB(n,k,z[1],z[2], . . ,z[n -k+1]) no MAPLE.

Aplicando (2.27) à equação (2.26) e trocando a ordem da soma e da integral, obtemos por integração simples

$$
\begin{aligned}
M(t) & =\exp \left(\eta_{0} t\right) \int_{0}^{1} \sum_{n, k=0}^{\infty} \frac{B_{n, k}}{n !} u^{n} t^{k} d u \\
& =\exp \left(\eta_{0} t\right) \sum_{n, k=0}^{\infty} \frac{B_{n, k}}{n !} t^{k} \int_{0}^{1} u^{n} d u \\
& =\exp \left(\eta_{0} t\right) \sum_{n, k=0}^{\infty} \frac{B_{n, k}}{(n+1) n !} t^{k} \\
& =\exp \left(\eta_{0} t\right) \sum_{n, k=0}^{\infty} \frac{B_{n, k} t^{k}}{(n+1) !}=\exp \left(\eta_{0} t\right) \sum_{k=0}^{\infty} s_{k} t^{k} .
\end{aligned}
$$


A equação (2.28) dá a fgm de $Z$ como uma função exponencial multiplicada por um polinômio infinito em $t$. É o resultado principal desta seção. Para a maioria das aplicações práticas, o grau mais alto do polinômio em (2.28) poderia ser 6 .

A função geradora acumulada (fga) de $Z$ é dada por $K(t)=\log [M(t)]$. As aproximações do ponto de sela são as principais aplicações da fga em Estatística e fornecem fórmulas de aproximação altamente precisas para a função densidade da soma e da média de variáveis aleatórias independentes e identicamente distribuídas (iid). Sejam $Z_{1}, \ldots, Z_{n}$ variáveis aleatórias $\mathrm{KwN}$ iid com fga comum $K(t)$. Seja $S_{n}=\sum_{i=1}^{n} Z_{i}$ e $K^{(j)}(\lambda)=d^{j} K(\lambda) / d \lambda^{j}$ (para $j \geq 1$ ). Definimos $\hat{\lambda}$ a partir da equação (não linear usual) $K^{(1)}(s, \hat{\lambda})=s / n$ e $z=\left\{s-n K^{(1)}(\lambda)\right\} / \sqrt{n K^{(2)}(\lambda)}$.

As funções de densidade de $S_{n}$ e $\bar{Z}_{n}=S_{n} / n$ podem ser expressas como aproximações de ponto de sela de Daniels dadas por

$$
f_{S_{n}}(s)=\frac{\exp \{n K(\hat{\lambda})-s \hat{\lambda}\}}{\sqrt{2 n \pi K^{(2)}(\hat{\lambda})}}\left\{1+N(\hat{\lambda})+O\left(n^{-2}\right)\right\}
$$

e

$$
f_{\bar{Z}_{n}}(z)=\left\{\frac{n}{2 \pi K^{(2)}(\hat{\lambda})}\right\}^{1 / 2} \exp [n\{K(\hat{\lambda})-\hat{\lambda} z\}]\left\{1+M(\hat{\lambda})+O\left(n^{-2}\right)\right\}
$$

em que

$$
N(\lambda)=\frac{3 \gamma_{2}(\lambda)-5 \gamma_{1}(\lambda)^{2}}{24 n}
$$

$\gamma_{1}(\lambda)=K^{(3)}(\lambda) / K^{(2)}(\lambda)^{3 / 2}$ e $\gamma_{2}(\lambda)=K^{(4)}(\lambda) / K^{(2)}(\lambda)^{2}$ são o terceiro e o quarto cumulantes padronizados de $Z$, respectivamente. O leitor que desejar mais detalhes pode consultar o livro de Cordeiro (1999, Seção 3.5). As aproximações para $f_{S_{n}}(s)$ e $f_{\bar{Z}_{n}}(z)$ normalmente darão bons resultados na prática.

\subsection{Momentos incompletos}

Definição 2.5. O r-ésimo momento incompleto de uma variável aleatória $Y$ é definido por

$$
m_{r}(q)=\int_{-\infty}^{q} y^{r} f(y) d y
$$

Vamos obter os momentos incompletos da variável aleatória $Z \sim \operatorname{KwN}(a, b, 0,1$,$) .$

Com base na equação (2.24), podemos escrever

$$
m_{r}(q)=\int_{0}^{F(q)}\left(\sum_{j=0}^{\infty} \eta_{j} u^{j}\right)^{r} d u
$$

Usando (2.18) e após a integração, obtemos

$$
m_{r}(q)=\int_{0}^{F(q)}\left(\sum_{j=0}^{\infty} \eta_{j} u^{j}\right)^{r} d u=\sum_{j=0}^{\infty} \frac{\delta_{r, j}}{j+1} F(q)^{j+1}
$$


em que (para $r \geq 0) \delta_{r, 0}=\eta_{0}^{r}, \delta_{r, j}=\left(r \eta_{0}\right)^{-1} \sum_{n=1}^{j}[n(r+1)-j] \eta_{n} \delta_{r, j-n}$, para $j \geq 1$.

A dispersão de $Z$ pode ser avaliada pela totalidade dos desvios em relação à média e à mediana. Esses desvios são conhecidos como desvios médios em torno da média $\left(\delta_{1}=E\left|Z-\mu_{1}^{\prime}\right|\right)$ e da mediana $\left(\delta_{2}=E|Z-M|\right)$, em que $\mu_{1}^{\prime}=E(Z)$ é a média e $M=Q(0.5)=\Phi^{-1}\left(\left[1-0.5^{1 / b}\right]^{1 / a}\right)$ é a mediana. Eles podem ser expressos em termos do primeiro momento incompleto, dados por

$$
\delta_{1}=2 \mu_{1}^{\prime} F\left(\mu_{1}^{\prime}\right)-2 m_{1}\left(\mu_{1}^{\prime}\right) \quad \text { e } \quad \delta_{2}=\mu_{1}^{\prime}-2 m_{1}(M)
$$

respectivamente, em que $m_{1}(q)$ é obtido a partir de (2.29) com $r=1$.

\subsection{Momentos ponderados por probabilidade}

$\mathrm{O}(r, j)$-ésimo momento ponderado por probabilidade (PWM), para $r$ e $j$ inteiros positivos, da distribuição KwN padrão, denotado por $\rho_{r, j}$, é dado por

$$
\rho_{r, j}=\int_{-\infty}^{\infty} x^{r} F(x)^{j} f(x) d x
$$

A partir da equação (2.8), obtemos

$$
F(x)=\Phi(x)^{a} \sum_{m=0}^{\infty} e_{m} \Phi(x)^{m a}
$$

em que $e_{m}=(-1)^{m+1}\left(\begin{array}{c}b \\ m+1\end{array}\right)$.

Então, usando (2.18), podemos escrever

$$
F(x)^{j}=\Phi(x)^{j a}\left(\sum_{m=0}^{\infty} e_{m} \Phi(x)^{m a}\right)^{j}=\sum_{m=0}^{\infty} f_{j, m} \Phi(x)^{(j+m) a}
$$

em que $f_{j, m}$ (para $m=1,2, \ldots$ ) segue recursivamente a partir de $f_{j, m}=\left(j e_{0}\right)^{-1} \sum_{n=1}^{m}[n(j+1)-$ $m] e_{n} f_{j, m-n}($ para $m \geq 1)$ e de $f_{j, 0}=e_{0}^{j}$.

Além disso, obtemos de (2.9)

$$
F(x)^{j}=\sum_{k=0}^{\infty} \kappa_{k, j} \Phi(x)^{k}
$$

em que $\kappa_{k, j}=\sum_{m=0}^{\infty} f_{j, m} s_{k}((j+m) a)$ (para $k \geq 0$ e $\left.j \geq 1\right)$.

Inserindo (2.10) e (2.31) na equação (2.30), obtemos

$$
\begin{aligned}
\rho_{r, j} & =\int_{-\infty}^{\infty} x^{r} \sum_{k=0}^{\infty} \kappa_{k, j} \Phi(x)^{k} \sum_{m=0}^{\infty} \phi(x) v_{m} \Phi(x)^{m} d u \\
& =\sum_{k=0}^{\infty} \sum_{m=0}^{\infty} \kappa_{k, j} v_{m} \int_{-\infty}^{\infty} x^{r} \phi(x) \Phi(x)^{k+m} d u \\
& =\sum_{k, m=0}^{\infty} \kappa_{k, j} v_{m} Q_{S N}(u)^{r} u^{k+m} d u
\end{aligned}
$$


Fazendo $u=\Phi(x)$, podemos rescrever $\rho_{r, j}$ como

$$
\begin{aligned}
\rho_{r, j} & =\sum_{k=0}^{\infty} \sum_{m=0}^{\infty} \kappa_{k, j} v_{m} \int_{0}^{1} Q_{S N}(u)^{r} u^{k+m} d u \\
& =\sum_{k, m=0}^{\infty} \kappa_{k, j} v_{m} \int_{0}^{1} Q_{S N}(u)^{r} u^{k+m} d u .
\end{aligned}
$$

Como provado logo após a equação (2.21), podemos escrever $Q_{S N}(u)^{r}=\sum_{n=0}^{\infty} f_{r, n} v^{n}$. Assim,

$$
\begin{aligned}
\rho_{r, j} & =\sum_{k, m=0}^{\infty} \sum_{n=0}^{\infty} \kappa_{k, j} v_{m} f_{r, n} \int_{0}^{1}[\sqrt{2 \pi}(u-1 / 2)]^{n} u^{k+m} d u \\
& =\sum_{k, m, n=0}^{\infty} \kappa_{k, j} v_{m} f_{r, n}(\sqrt{2 \pi})^{n} \int_{0}^{1}(u-1 / 2)^{n} u^{k+m} d u \\
& =\sum_{k, m, n=0}^{\infty} \kappa_{k, j} v_{m} f_{r, n}(\sqrt{2 \pi})^{n} \sum_{s=0}^{\infty} \frac{(-1 / 2)^{n-s}}{s+k+m+1}\left(\begin{array}{c}
n \\
s
\end{array}\right) .
\end{aligned}
$$

Finalmente, obtém-se

$$
\rho_{r, j}=\sum_{k, m, n, s=0}^{\infty} \frac{(-1 / 2)^{n-s}(\sqrt{2 \pi})^{n} \kappa_{k, j} v_{m} f_{r, n}}{s+k+m+1}\left(\begin{array}{l}
n \\
s
\end{array}\right)
$$

O limite infinito, no exemplo acima, pode ser alterado por um número nem tão elevado como 20.

\subsection{Estatísticas de ordem}

Suponha que $Z_{1}, \ldots, Z_{n}$ seja uma amostra aleatória da distribuição KwN padrão e sejam $Z_{1: n}<\cdots<Z_{n: n}$ as estatísticas de ordem correspondentes. Segundo Cordeiro e de Castro (2011), podemos escrever a função densidade $f_{i: n}(x)$ da $i$-ésima estatística de ordem $Z_{i: n}$ (para $i=1, \ldots, n$ ) como

$$
f_{i: n}(x)=\phi(x) \sum_{r, k=0}^{\infty} q_{r, k} \Phi(x)^{a(k+1)+r-1},
$$

em que

$$
q_{r, k}=\sum_{j=0}^{n-i} \frac{(-1)^{j}\left(\begin{array}{c}
n-i \\
j
\end{array}\right) w_{k} p_{r, i+j-1}}{B(i, n-i+1)},
$$

e as quantidades $p_{r, u}(a, b)$ (para $\left.r, u=0,1, \ldots\right)$ são dadas por

$$
p_{r, u}(a, b)=\sum_{k=0}^{u}(-1)^{k}\left(\begin{array}{l}
u \\
k
\end{array}\right) \sum_{m=0}^{\infty} \sum_{l=r}^{\infty}(-1)^{m r+l}\left(\begin{array}{l}
k b \\
m
\end{array}\right)\left(\begin{array}{c}
m a \\
l
\end{array}\right)\left(\begin{array}{l}
l \\
r
\end{array}\right) .
$$


Usando a equação (2.9), podemos reescrever (2.32) como

$$
\begin{aligned}
f_{i: n}(x) & =\phi(x) \sum_{r, k=0}^{\infty} q_{r, k} \sum_{j=0}^{\infty} s_{j}[a(k+1)+r-1] \Phi(x)^{j} \\
& =\phi(x) \sum_{j=0}^{\infty} \sum_{r, k=0}^{\infty} q_{r, k} s_{j}[a(k+1)+r-1] \Phi(x)^{j} \\
& =\phi(x) \sum_{j=0}^{\infty} \pi_{j} \Phi(x)^{j},
\end{aligned}
$$

em que $\pi_{j}=\sum_{r, k=0}^{\infty} q_{r, k} s_{j}[a(k+1)+r-1]$ e $s_{j}[a(k+1)+r-1]$ é dado em (2.9).

A equação (2.33) para a função densidade das estatísticas de ordem KwN é o principal resultado desta seção. Seguindo resultados semelhantes aos das Seções 3.3 e 3.4, pode-se obter os momentos ordinários, incompletos e função geradora de momentos das estatísticas de ordem KwN. Por exemplo, o $r$-ésimo momento de $Z_{i: n}$ vem da equação (2.33) como

$$
\begin{aligned}
E\left(Z_{i: n}^{r}\right) & =\int_{-\infty}^{\infty} x^{r} \phi(x) \sum_{j=0}^{\infty} \pi_{j} \Phi(x)^{j} d x \\
& =\sum_{j=0}^{\infty} \pi_{j} \int_{-\infty}^{\infty} x^{r} \phi(x) \Phi(x)^{j} d x \\
& =\sum_{j=0}^{\infty} \pi_{j} \tau_{r, j},
\end{aligned}
$$

em que $\tau_{r, j}$ é facilmente obtido a partir da equação (2.17). Então, os momentos ordinários das estatísticas de ordem de $Z$ são funções lineares simples dos PWMs das distribuições normais.

\subsection{Entropias}

A entropia é uma medida de incerteza associada a uma variável aleatória. A entropia de uma variável aleatória $X$ é definida em termos de sua função de densidade $f(x)$. Considere uma variável aleatória $X \sim \operatorname{KwN}(a, b, \mu, \sigma)$.

Proposição 2.4. A entropia de Shannon é dada por

$$
\begin{aligned}
I_{S}(X)= & \log \left(\frac{\sqrt{2 \pi}}{a b}\right)+\frac{1}{2 \sigma^{2}}\left[E\left(X^{2}\right)-2 \mu E(X)+\mu^{2}\right]-a b \sum_{n=1}^{\infty} \sum_{k=0}^{\infty}\left(\begin{array}{c}
b-1 \\
k
\end{array}\right) \frac{(-1)^{n+k}}{n} \times \\
& {\left[(a-1) B(a(k+1), n+1)+\frac{(b-1)}{a(n+k+1)}\right] . }
\end{aligned}
$$

Demonstração. A medida de entropia de Shannon é definida por

$$
I_{S}(X)=-E[\log f(X)]
$$

A partir da função densidade da distribuição $\operatorname{KwN}\left(a, b, \mu, \sigma^{2}\right)$, temos 


$$
\begin{aligned}
I_{S}(X)= & -\int_{-\infty}^{\infty} f(x) \log f(x) d x \\
= & -\log \left(\frac{a b}{\sigma}\right)-(a-1) \int_{-\infty}^{\infty} f(x) \log \left[\Phi\left(\frac{x-\mu}{\sigma}\right)\right] d x- \\
& -(b-1) \int_{-\infty}^{\infty} f(x) \log \left[1-\Phi\left(\frac{x-\mu}{\sigma}\right)^{a}\right] d x-\int_{-\infty}^{\infty} f(x) \log \left[\phi\left(\frac{x-\mu}{\sigma}\right)\right] d x .
\end{aligned}
$$

A primeira das três integrais pode ser expressa em termos da função beta. Usando expansão em série de Taylor para a função logarítmica, obtemos

$$
\log \left[1-\Phi\left(\frac{x-\mu}{\sigma}\right)\right]=\sum_{n=1}^{\infty} \frac{(-1)^{n}}{n} \Phi\left(\frac{x-\mu}{\sigma}\right)^{n}
$$

e

$$
\log \left\{1-\left[1-\Phi\left(\frac{x-\mu}{\sigma}\right)\right]\right\}=\sum_{n=1}^{\infty} \frac{(-1)^{n}}{n}\left[1-\Phi\left(\frac{x-\mu}{\sigma}\right)\right]^{n} .
$$

Consequentemente, as integrais anteriores podem ser escritas como

$$
\begin{aligned}
\int_{-\infty}^{\infty} f(x) \log \left[\Phi\left(\frac{x-\mu}{\sigma}\right)\right] d x= & \sum_{n=1}^{\infty} \frac{(-1)^{n}}{n} \int_{-\infty}^{\infty} f(x)\left[1-\Phi\left(\frac{x-\mu}{\sigma}\right)\right]^{n} d x \\
= & \frac{a b}{\sigma} \sum_{n=1}^{\infty} \frac{(-1)^{n}}{n} \int_{-\infty}^{\infty}\left[1-\Phi\left(\frac{x-\mu}{\sigma}\right)\right]^{n} \\
& \times\left[\Phi\left(\frac{x-\mu}{\sigma}\right)\right]^{a-1}\left[1-\Phi\left(\frac{x-\mu}{\sigma}\right)^{a}\right]^{b-1} \phi\left(\frac{x-\mu}{\sigma}\right) \\
= & \frac{a b}{\sigma} \sum_{n=1}^{\infty} \sum_{k=0}^{\infty}\left(\begin{array}{c}
b-1 \\
k
\end{array}\right) \frac{(-1)^{n+k}}{n} \int_{-\infty}^{\infty} \phi\left(\frac{x-\mu}{\sigma}\right) \\
& \times \Phi\left(\frac{x-\mu}{\sigma}\right)^{a(k+1)-1}\left[1-\Phi\left(\frac{x-\mu}{\sigma}\right)\right]^{n} d x .
\end{aligned}
$$

Na última igualdade, fazendo a mudança de variável $u=\Phi\left(\frac{x-\mu}{\sigma}\right)$ obtemos

$$
\begin{aligned}
\int_{-\infty}^{\infty} f(x) \log \left[\Phi\left(\frac{x-\mu}{\sigma}\right)\right] d x & =\sum_{n=1}^{\infty} \sum_{k=0}^{\infty}\left(\begin{array}{c}
b-1 \\
k
\end{array}\right) \frac{(-1)^{n+k}}{n} \int_{0}^{1} u^{a(k+1)-1}(1-u)^{n} d u \\
& =a b \sum_{n=1}^{\infty} \sum_{k=0}^{\infty}\left(\begin{array}{c}
b-1 \\
k
\end{array}\right) \frac{(-1)^{n+k}}{n} B(a(k+1), n+1) .
\end{aligned}
$$

Analogamente,

$$
\int_{-\infty}^{\infty} f(x) \log \left[1-\Phi\left(\frac{x-\mu}{\sigma}\right)^{a}\right] d x=\sum_{n=1}^{\infty} \frac{(-1)^{n}}{n} \int_{-\infty}^{\infty} f(x)\left[\Phi\left(\frac{x-\mu}{\sigma}\right)\right]^{a n} d x
$$




$$
\begin{aligned}
\int_{-\infty}^{\infty} f(x) \log \left[1-\Phi\left(\frac{x-\mu}{\sigma}\right)^{a}\right] d x= & \frac{a b}{\sigma} \sum_{n=1}^{\infty} \sum_{k=0}^{\infty} \frac{(-1)^{n+k}}{n}\left(\begin{array}{c}
b-1 \\
k
\end{array}\right) \\
& \times \int_{-\infty}^{\infty} \Phi\left(\frac{x-\mu}{\sigma}\right)^{a(n+k+1)-1} \phi\left(\frac{x-\mu}{\sigma}\right) d x \\
= & a b \sum_{n=1}^{\infty} \sum_{k=0}^{\infty} \frac{(-1)^{n+k}}{n}\left(\begin{array}{c}
b-1 \\
k
\end{array}\right) \\
& \times \int_{0}^{1} u^{a(n+k+1)-1} d u \\
= & a b \sum_{n=1}^{\infty} \sum_{k=0}^{\infty} \frac{(-1)^{n+k}}{n a(n+k+1)}\left(\begin{array}{c}
b-1 \\
k
\end{array}\right) .
\end{aligned}
$$

Podemos escrever a última integral como

$$
\begin{aligned}
\int_{-\infty}^{\infty} f(x) \log \phi\left(\frac{x-\mu}{\sigma}\right) d x & =\int_{-\infty}^{\infty} f(x)\left\{\log \left(\frac{1}{\sqrt{2 \pi}}\right)+\log \left[\exp \left(-\frac{(x-\mu)^{2}}{2 \sigma^{2}}\right)\right]\right\} d x \\
& =-\log (\sqrt{2 \pi})-\frac{1}{2 \sigma^{2}}\left[E\left(X^{2}\right)-2 \mu E(X)+\mu^{2}\right]
\end{aligned}
$$

em que $E\left(X^{2}\right)$ e $E(X)$ seguem dos resultados da Seção 3.3. Finalmente, a entropia de Shannon é dada por

$$
\begin{aligned}
I_{S}(X)= & \log \left(\frac{\sqrt{2 \pi}}{a b}\right)+\frac{1}{2 \sigma^{2}}\left[E\left(X^{2}\right)-2 \mu E(X)+\mu^{2}\right]-a b \sum_{n=1}^{\infty} \sum_{k=0}^{\infty}\left(\begin{array}{c}
b-1 \\
k
\end{array}\right) \frac{(-1)^{n+k}}{n} \\
& \times\left[(a-1) B(a(k+1), n+1)+\frac{(b-1)}{a(n+k+1)}\right] .
\end{aligned}
$$

Proposição 2.5. A entropia de Rényi é dada por

$$
\begin{aligned}
\mathcal{J}_{R}(c)= & \frac{1}{1-c} \log \left[\frac{(a b)^{c}}{(\sqrt{2 \pi})^{c-1} \sigma^{2 c-1}}\right]+\frac{1}{1-c} \log \left[\sum_{r=0}^{\infty} \sum_{j=0}^{s} \sum_{k_{1}, \ldots, k_{j}=0}^{\infty} \frac{S_{r}(c)}{2^{s}(\sqrt{c})^{2 m_{j}+j}}\left(\begin{array}{l}
s \\
j
\end{array}\right)\right. \\
& \left.\times A\left(k_{1}, \ldots, k_{j}\right) \frac{\left(2 m_{j}+j\right) !}{2^{\left(2 m_{j}+j\right) / 2}\left[\left(2 m_{j}+j\right) / 2\right] !} \delta_{\{2,4,6, \ldots\}}\left(2 m_{j}+j\right)\right],
\end{aligned}
$$

em que $S_{r}(c)=(-1)^{r}\left(\begin{array}{c}(b-1) c \\ r\end{array}\right)$.

Demonstração. A medida de entropia de Rényi é definida por

$$
\mathcal{J}_{R}(c)=\frac{1}{1-c} \log \left[\int_{-\infty}^{\infty} f^{c}(x) d x\right], c>0, c \neq 1
$$

Observe que quando $c \rightarrow 1$, a entropia de Rényi converge para a entropia de Shannon. A partir da função densidade da distribuição $\operatorname{KwN}\left(a, b, \mu, \sigma^{2}\right)$, temos

$$
f^{c}(x)=\left(\frac{a b}{\sqrt{2 \pi} \sigma^{2}}\right)^{c}\left[\Phi\left(\frac{x-\mu}{\sigma}\right)\right]^{(a-1) c}\left[1-\Phi\left(\frac{x-\mu}{\sigma}\right)^{a}\right]^{(b-1) c} \exp \left[-\frac{c}{2}\left(\frac{x-\mu}{\sigma}\right)^{2}\right] .
$$


Ao expandir o binômio e alterar termos, obtemos

$$
\begin{aligned}
f^{c}(x) & =\left(\frac{a b}{\sqrt{2 \pi} \sigma^{2}}\right)^{c} \exp \left[-\frac{c}{2}\left(\frac{x-\mu}{\sigma}\right)^{2}\right] \sum_{r=0}^{\infty}(-1)^{r}\left(\begin{array}{c}
(b-1) c \\
r
\end{array}\right) \Phi\left(\frac{x-\mu}{\sigma}\right)^{a r+c(a-1)} \\
& =C(x) \sum_{r=0}^{\infty} S_{r}(c) \Phi\left(\frac{x-\mu}{\sigma}\right)^{a r+c(a-1)}
\end{aligned}
$$

em que $S_{r}(c)=(-1)^{r}\left(\begin{array}{c}(b-1) c \\ r\end{array}\right)$ e

$$
C(x)=\left(\frac{a b}{\sqrt{2 \pi} \sigma^{2}}\right)^{c} \exp \left[-\frac{c}{2}\left(\frac{x-\mu}{\sigma}\right)^{2}\right] .
$$

Então, a integral em (2.35) pode ser expressa como

$$
\begin{aligned}
\int_{-\infty}^{\infty} f^{c}(x) d x & =\sum_{r=0}^{\infty} S_{r}(c) \int_{-\infty}^{\infty} C(x) \Phi\left(\frac{x-\mu}{\sigma}\right)^{a r+c(a-1)} d x \\
& =\left(\frac{a b}{\sqrt{2 \pi} \sigma^{2}}\right)^{c} \sum_{s=0}^{\infty} S_{r}(c) \int_{-\infty}^{\infty} \exp \left[-\frac{c}{2}\left(\frac{x-\mu}{\sigma}\right)^{2}\right] \Phi\left(\frac{x-\mu}{\sigma}\right)^{a r+c(a-1)} d x
\end{aligned}
$$

Seja

$$
A_{R}(c)=\int_{-\infty}^{\infty} \exp \left[-\frac{c}{2}\left(\frac{x-\mu}{\sigma}\right)^{2}\right] \Phi\left(\frac{x-\mu}{\sigma}\right)^{a r+c(a-1)} d x
$$

A entropia de Rényi pode ser determinada como

$$
\mathcal{J}_{R}(c)=\frac{1}{1-c} \log \left[\left(\frac{a b}{\sqrt{2 \pi} \sigma^{2}}\right)^{c} \sum_{r=0}^{\infty} S_{r}(c) A_{R}(c)\right] .
$$

Fixando $t=(x-\mu) / \sigma, A_{R}(c)$ se reduz a

$$
A_{R}(c)=\sigma \int_{-\infty}^{\infty} \exp \left(-\frac{c t^{2}}{2}\right) \Phi(t)^{s} d t
$$

em que $s=a r+c(a-1)$.

Combinando as equações (2) e (9) de Castellares et al. (2013), $A_{R}(c)$ pode ser escrita como

$$
A_{R}(c)=\frac{\sigma}{2^{s}} \sum_{j=0}^{s} \sum_{k_{1}, \ldots, k_{j}=0}\left(\begin{array}{l}
s \\
j
\end{array}\right) A\left(k_{1}, \ldots, k_{j}\right) \int_{-\infty}^{\infty} t^{2 m_{j}+j} \exp \left(-\frac{c t^{2}}{2}\right) d t
$$

$\operatorname{com} a_{k}=\left[(-1)^{k} 2^{(1-2 k) / 2}\right] /(\sqrt{\pi}(2 k+1) k !), A\left(k_{1}, \ldots, k_{j}\right)=a_{k_{1}} \ldots a_{k_{j}}$ e $m_{j}=k_{1}+\ldots+k_{j}$.

Definindo a integral

$$
J_{c}\left(m_{j}+j\right)=\int_{-\infty}^{\infty} t^{2 m_{j}+j} \exp \left(-\frac{(\sqrt{c} t)^{2}}{2}\right) d t
$$


podemos reescrever $A_{R}(c)$ como

$$
A_{R}(c)=\frac{\sigma}{2^{s}} \sum_{j=0}^{s} \sum_{k_{1}, \ldots, k_{j}=0}^{\infty}\left(\begin{array}{l}
s \\
j
\end{array}\right) A\left(k_{1}, \ldots, k_{j}\right) J_{c}\left(m_{j}+j\right) .
$$

Fazendo $u=\sqrt{c} t$, obtemos

$$
J_{c}\left(m_{j}+j\right)=\frac{\sqrt{2 \pi}}{(\sqrt{c})^{2 m_{j}+j}} \int_{-\infty}^{\infty} u^{2 m_{j}+j} \frac{1}{\sqrt{2 \pi}} \exp \left(-\frac{u^{2}}{2}\right) d u
$$

Usando o $\left(2 m_{j}+j\right)$-ésimo momento da variável aleatória normal padrão, temos

$$
J_{c}\left(m_{j}+j\right)=\frac{\sqrt{2 \pi}}{(\sqrt{c})^{2 m_{j}+j}} \frac{\left(2 m_{j}+j\right) !}{2^{\left(2 m_{j}+j\right) / 2}\left[\left(2 m_{j}+j\right) / 2\right] !} \delta_{\{2,4,6, \ldots\}}\left(2 m_{j}+j\right),
$$

em que

$$
\delta_{A}(l)= \begin{cases}1, \text { se } & l \in A, \\ 0, \text { se } & l \notin A .\end{cases}
$$

Finalmente, inserindo (2.37) em (2.35), obtemos

$$
\begin{aligned}
\mathcal{J}_{R}(c)= & \frac{1}{1-c} \log \left[\frac{(a b)^{c}}{(\sqrt{2 \pi})^{c-1} \sigma^{2 c-1}}\right]+\frac{1}{1-c} \log \left[\sum_{r=0}^{\infty} \sum_{j=0}^{s} \sum_{k_{1}, \ldots, k_{j}=0}^{\infty} \frac{S_{r}(c)}{2^{s}(\sqrt{c})^{2 m_{j}+j}}\left(\begin{array}{l}
s \\
j
\end{array}\right)\right. \\
& \left.\times A\left(k_{1}, \ldots, k_{j}\right) \frac{\left(2 m_{j}+j\right) !}{2^{\left(2 m_{j}+j\right) / 2}\left[\left(2 m_{j}+j\right) / 2\right] !} \delta_{\{2,4,6, \ldots\}}\left(2 m_{j}+j\right)\right] .
\end{aligned}
$$

\subsection{Estimação}

Várias abordagens para a estimação pontual de parâmetros foram propostas na literatura. Dentre as mais conhecidas temos, o método dos momentos, o método dos mínimos quadrados e o método de máxima verossimilhança, sendo o método de máxima verossimilhança o mais comumente empregado. Os estimadores de máxima verossimilhança (EMV) possuem propriedades desejáveis que podem ser usadas, por exemplo, para construir intervalos de confiança para os parâmetros de um modelo. Nesta seção, vamos determinar os EMV dos parâmetros da distribuição $\operatorname{KwN}(a, b, \mu, \sigma)$.

Considere $X_{1}, \ldots, X_{n}$ uma amostra aleatória de tamanho $n$ da distribuição $\mathrm{KwN}\left(a, b, \mu, \sigma^{2}\right)$. A função de verossimilhança para o vetor de parâmetros $\boldsymbol{\theta}=\left(a, b, \mu, \sigma^{2}\right)^{\top}$ é dada por

$$
\begin{aligned}
L(\boldsymbol{\theta}) & =\prod_{i=1}^{n} f\left(x_{i}, \boldsymbol{\theta}\right) \\
& =\left(\frac{a b}{\sigma^{2} \sqrt{2 \pi}}\right)^{n} \prod_{i=1}^{n}\left[\Phi\left(z_{i}\right)\right]^{a-1} \prod_{i=1}^{n}\left[1-\Phi\left(z_{i}\right)^{a}\right]^{b-1} \prod_{i=1}^{n} \exp \left(-\frac{z_{i}^{2}}{2}\right),
\end{aligned}
$$

em que $z_{i}=\left(x_{i}-\mu\right) / \sigma$. 
A função de log-verossimilhança pode ser expressa como

$$
\begin{aligned}
\ell(\boldsymbol{\theta})= & n \log (a)+n \log (b)-n \log \left(\sigma^{2}\right)-\frac{n}{2} \log (2 \pi)+\sum_{i=1}^{n}\left\{(a-1) \log \left[\Phi\left(z_{i}\right)\right]\right. \\
& \left.+(b-1) \log \left[1-\Phi\left(z_{i}\right)^{a}\right]-\frac{z_{i}^{2}}{2}\right\} .
\end{aligned}
$$

A equação (2.39) pode ser maximizada diretamente usando a linguagem $R$ (função optim), SAS (PROC NLMIXED) ou Ox (função MaxBFGS).

Então, os componentes do vetor escore $U(\boldsymbol{\theta})$ são dados por

$$
\begin{aligned}
U_{a}(\boldsymbol{\theta}) & =\frac{n}{a}+\sum_{i=1}^{n}\left\{\log \left[\Phi\left(z_{i}\right)\right]-\frac{(b-1)\left[\Phi\left(z_{i}\right)\right]^{a} \log \left[\Phi\left(z_{i}\right)\right]}{1-\Phi\left(z_{i}\right)^{a}}\right\}, \\
U_{b}(\boldsymbol{\theta}) & =\frac{n}{b}+\sum_{i=1}^{n} \log \left[1-\Phi\left(z_{i}\right)^{a}\right], \\
U_{\mu}(\boldsymbol{\theta}) & =\sum_{i=1}^{n} \frac{1}{\sigma}\left\{z_{i}-\frac{(a-1) \phi\left(z_{i}\right)}{\Phi\left(z_{i}\right)}+\frac{a(b-1)\left[\Phi\left(z_{i}\right)\right]^{a-1} \phi\left(z_{i}\right)}{1-\Phi\left(z_{i}\right)^{a}}\right\},
\end{aligned}
$$

e

$$
U_{\sigma}(\boldsymbol{\theta})=-\frac{2 n}{\sigma}+\frac{1}{\sigma} \sum_{i=1}^{n}\left\{z_{i}^{2}-z_{i} \phi\left(z_{i}\right)\left[\frac{(a-1)}{\Phi\left(z_{i}\right)}-\frac{a(b-1)\left[\Phi\left(z_{i}\right)\right]^{a-1}}{1-\Phi\left(z_{i}\right)^{a}}\right]\right\} .
$$

Sob certas condições de regularidade, segue o resultado assintótico

$$
\widehat{\boldsymbol{\theta}}-\boldsymbol{\theta} \sim N_{4}\left(0, K(\boldsymbol{\theta})^{-1}\right)
$$

em que $K(\boldsymbol{\theta})$ é a matriz de informação esperada.

A distribuição normal multivariada $N_{4}\left(0, K(\boldsymbol{\theta})^{-1}\right)$ pode ser usada para construir intervalos de confiança aproximados e regiões de confiança para os parâmetros do modelo. Quando a obtenção de $K(\boldsymbol{\theta})$ é difícil, essa matriz pode ser aproximada pela matriz de informação observada $J(\boldsymbol{\theta})$ dada por

$$
J(\boldsymbol{\theta})=\left[\begin{array}{cccc}
J_{a a} & J_{a b} & J_{a \mu} & J_{a \sigma} \\
J_{b a} & J_{b b} & J_{b \mu} & J_{b \sigma} \\
J_{\mu a} & J_{\mu b} & J_{\mu \mu} & J_{\mu \sigma} \\
J_{\sigma a} & J_{\sigma b} & J_{\sigma \mu} & J_{\sigma \sigma}
\end{array}\right],
$$

em que os elementos da matriz $J(\boldsymbol{\theta})$ são dados no Apêndice A.

A comparação da distribuição KwN com alguns de seus modelos especiais pode ser baseada em estatísticas da razão de verossimilhanças (RV). Podemos calcular os valores máximos da função log-verossimilhança irrestrita e restrita para construir a estatística RV para testar alguns submodelos da distribuição KwN. Por exemplo, o teste de $H_{0}: a=b=1$ versus $H_{1}: H_{0}$ não é verdadeira é equivalente a comparar as distribuições KwN e normal e então a estatística RV reduz a $w=2\{\ell(\hat{a}, \hat{b}, \hat{\mu}, \hat{\sigma})-\ell(1,1, \tilde{\mu}, \tilde{\sigma})\}$, em que $\hat{a}, \hat{b}, \hat{\mu}$ e $\hat{\sigma}$ são os EMVs sob $H_{1}$ e $\tilde{\mu}$ e $\tilde{\sigma}$ são os estimadores sob $H_{0}$. A hipótese nula é rejeitada se $w>\chi_{1-\alpha}^{2}(1)$, em que $\chi_{1-\alpha}^{2}(1)$ é o quantil $1-\alpha$ da distribuição qui-quadrado com um grau de liberdade e $\alpha$ é o coeficiente de significância do teste . 


\subsection{Estudo de simulação}

Um estudo de simulação de Monte Carlo foi realizado para verificar o comportamento dos estimadores de máxima verosimilhança da distribuição KwN. As simulações são realizadas através da geração de observações a partir da distribuição KwN utilizando o método de transformação inversa. Foram geradas amostras com tamanhos $n=30, n=50, n=100$ e $n=200$ e os valores dos parâmetros foram fixados em $a=18, b=10, \mu=0,1$ e $\sigma=40$.

Para cada combinação de $n, a, b, \mu$ e $\sigma$, foram simuladas 2000 amostras. A Tabela 2.1 apresenta os valores das estimativas dos parâmetros e o erro quadrático médio (EQM) para cada tamanho amostral. Como podemos ver, os resultados são satisfatórios, ou seja, à medida que o tamanho da amostra aumenta, as estimativas são mais próximas aos valores verdadeiros de $a, b, \mu$ e $\sigma$ e os valores de EQM diminuem, na maioria dos casos, como era esperado.

Tabela 2.1: Média e EQM das estimativas dos parâmetros da distribuição KwN de acordo com o tamanho amostral.

\begin{tabular}{cccc}
\hline$n$ & Parâmetro & Média & EQM \\
\hline \multirow{3}{*}{30} & $a$ & 17,8737 & 0,0159 \\
& $b$ & 10,1014 & 0,0102 \\
& $\mu$ & 0,0005 & 0,0098 \\
& $\sigma$ & 39,8374 & 0,0264 \\
\hline \multirow{3}{*}{50} & $\mathrm{a}$ & 17.8912 & 0,0118 \\
& $\mathrm{~b}$ & 10,1025 & 0,0105 \\
& $\mu$ & 0.0006 & 0,0098 \\
& $\sigma$ & 39.8427 & 0,0350 \\
\hline \multirow{3}{*}{100} & $\mathrm{a}$ & 17,8727 & 0,0111 \\
& $\mathrm{~b}$ & 10,0816 & 0,0066 \\
& $\mu$ & 0,0007 & 0,0099 \\
& $\sigma$ & 39,8786 & 0,0147 \\
\hline \multirow{3}{*}{200} & $\mathrm{a}$ & 17,8929 & 0,0119 \\
& $\mathrm{~b}$ & 10,0037 & 0,0054 \\
& $\mu$ & 0,0008 & 0,0097 \\
& $\sigma$ & 39,8945 & 0,0105 \\
\hline
\end{tabular}

\subsection{Aplicação}

Nesta seção, usamos um conjunto de dados reais para ilustrar a utilidade da distribuição KwN. Estimamos os parâmetros desse modelo por máxima verossimilhança usando a linguagem R. A descrição do conjunto de dados é dada na Seção 2.12.1.

\subsubsection{Dados de monóxido de carbono}

Os dados referem-se a medições de monóxido de carbono $(\mathrm{CO})$ feitas em várias marcas de cigarros em 1994. Os dados foram coletados pela Comissão Federal do Comércio (CFC), uma agência independente do governo dos Estados Unidos, cuja principal missão é a promoção da proteção do consumidor. Durante três décadas, a CFC regularmente lançou relatórios sobre o teor de nicotina e alcatrão dos cigarros. Os relatórios indicam que os níveis de nicotina, em média, se mantiveram 
estáveis desde 1980. O endereço eletrônico http://www.ftc.gov/reports/tobacco inclui os conjuntos de dados e algumas informações sobre a fonte dos dados, o comportamento e as crenças dos fumantes acerca dos conteúdos de nicotina, alcatrão e monóxido de carbono nos cigarros. O conjunto de dados CO pode ser encontrado em http://home.att.net/ rdavis2 / cigra.html. Na Tabela 2.2, listamos algumas estatísticas resumo para os dados de CO. Pela Tabela 2.2 e Figura 2.4 notamos que esse conjunto de dados tem a mediana maior do que a média, que sugere que a distribuição é assimétrica, um fato reforçado pelo valor negativo do coeficiente de assimetria.

Tabela 2.2: Medidas descritivas

\begin{tabular}{lccccccc}
\hline Média & Mediana & Desvio padrão & Variância & Assimetria & Curtose & Mínimo & Máximo \\
\hline 11,34 & 12 & 4,06 & 16,55 & $-0,48$ & $-0,23$ & 0,05 & 22 \\
\hline
\end{tabular}

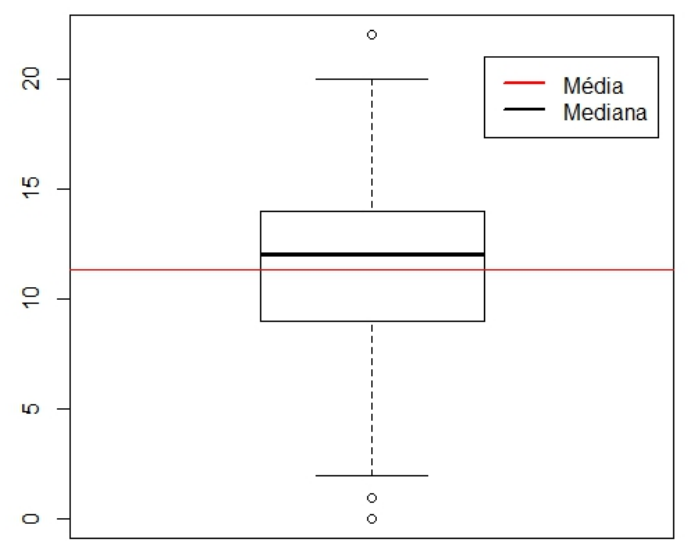

Figura 2.4: Gráfico boxplot para os dados de monóxido de carbono.

Apresentamos na Tabela 2.3 as estimativas de máxima verossimilhança dos parâmetros do modelo, seus erros padrão estimados e os valores dos critérios de informação AIC (critério de informação de Akaike), BIC (critério de informação bayesiano) e CAIC (critério de informação de Akaike corrigido). Os critérios de informação AIC, BIC e CAIC apresentam os valores mais baixos para a distribuição KwN, o que indica que essa distribuição é mais apropriada para ajustar esses dados do que as distribuições normal, beta-normal, exponencial normal e skew normal. Os histogramas, as funções de densidade e distribuições estimadas das distribuições KwN e normal são exibidas na Figura 2.5.

Utilizamos a estatística da razão de verossimilhanças (RV) para testar os modelos encaixados. Os resultados são apresentados na Tabela 2.4. Observemos que rejeitamos a hipótese nula em todos os três testes da razão de verossimilhanças em favor da distribuição KwN. Observamos ainda que 
Tabela 2.3: Estimativas de máxima verossimilhança e respectivos erros padrões dos parâmetros para os modelos de regressão $K w N$, beta normal (BN), exponenciada normal (Exp-N) e normal ajustados aos dados de $C O$ e valores dos critérios de informação

\begin{tabular}{|c|c|c|c|c|c|c|c|}
\hline \multirow{2}{*}{ Distribuição } & \multicolumn{4}{|c|}{ Parâmetros } & \multicolumn{3}{|c|}{ Critérios } \\
\hline & $a$ & $b$ & $\mu$ & $\sigma$ & $\mathrm{AIC}$ & CAIC & $\mathrm{BIC}$ \\
\hline $\mathrm{KwN}$ & $\begin{array}{c}0,2242 \\
(0,0420)\end{array}$ & $\begin{array}{c}0,0730 \\
(0,0262)\end{array}$ & $\begin{array}{l}11,8209 \\
(1,1516)\end{array}$ & $\begin{array}{c}1,2921 \\
(0,1306)\end{array}$ & 1929,1 & 1929,2 & 1944,5 \\
\hline $\mathrm{BN}$ & $\begin{array}{c}0,2232 \\
(0,0906)\end{array}$ & $\begin{array}{c}3,1108 \\
(0,4851)\end{array}$ & $\begin{array}{l}18,4546 \\
(0,4680)\end{array}$ & $\begin{array}{c}2,9305 \\
(0,5866)\end{array}$ & 1932,9 & 1933,0 & 1948,3 \\
\hline Exp-N & $\begin{array}{c}0,08012 \\
(0,04323)\end{array}$ & $\begin{array}{l}1 \\
-\end{array}$ & $\begin{array}{c}17,24835 \\
(0,75514)\end{array}$ & $\begin{array}{c}1,57333 \\
(0,35754)\end{array}$ & 1934,5 & 1934,6 & 1946,0 \\
\hline Normal & $\begin{array}{l}1 \\
-\end{array}$ & $\begin{array}{l}1 \\
-\end{array}$ & $\begin{array}{l}11,3425 \\
(0,2181)\end{array}$ & $\begin{array}{c}4,0626 \\
(0,1544)\end{array}$ & 1950,3 & 1950,4 & 1958,1 \\
\hline & & $\lambda$ & $\mu$ & $\sigma$ & & & \\
\hline Skew Normal & & $\begin{array}{c}0,0063 \\
(1,6075)\end{array}$ & $\begin{array}{l}11,3222 \\
(5,2118)\end{array}$ & $\begin{array}{c}4,0626 \\
(0,1562)\end{array}$ & 1952,3 & 1952,4 & 1963,8 \\
\hline
\end{tabular}

a rejeição é significativa, o que evidencia a necessidade dos parâmetros extras no modelo proposto na modelagem desses dados reais.

Tabela 2.4: Testes $R V$

\begin{tabular}{c|c|c|c}
\hline \hline Monóxido de Carbono & Hipóteses & Estatística $w$ & valor- $p$ \\
\hline \hline KwN versus BN(1,b) & $H_{0}: a=1$ versus $H_{1}: a \neq 1$ & 4,3 & 0,0042 \\
KwN versus Exp-N & $H_{0}: b=1$ versus $H_{1}: b \neq 1$ & 5,4 & 0,0003 \\
KwN versus Normal & $H_{0}: a=b=1$ versus $H_{1}: a \neq 1$ ou $b \neq 1$ & 23,2 & $<0,0001$ \\
\hline \hline
\end{tabular}
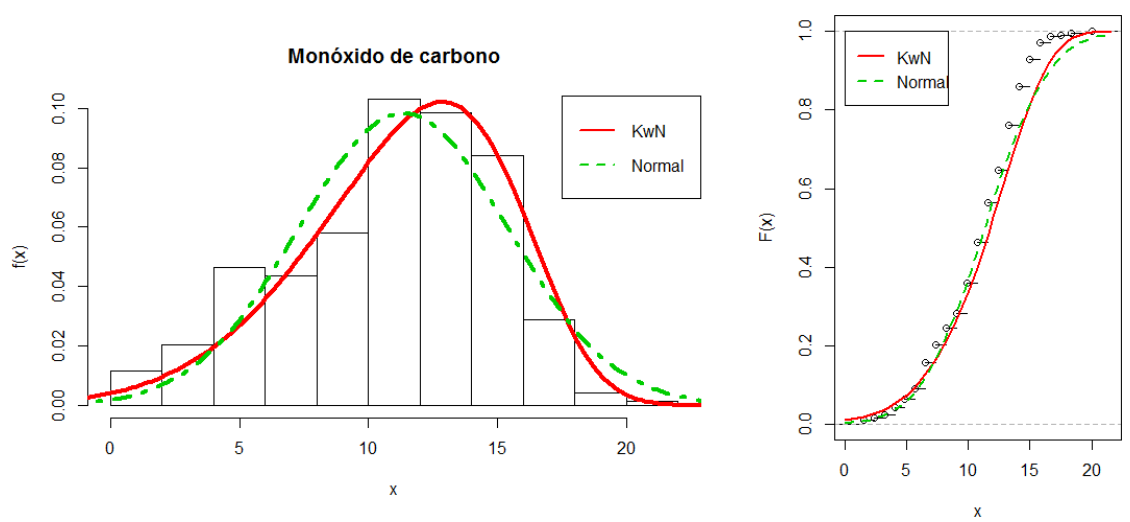

Figura 2.5: (a) Densidades estimadas dos modelos KwN e normal para os dados de monóxido de carbono. (b) Funções de distribuição acumuladas estimadas e a fda empírica para os dados de monóxido de carbono. 


\section{Capítulo 3}

\section{Modelo de regressão Kumaraswamy normal}

Neste capítulo apresentamos o modelo de regressão Kumaraswamy normal. Estimamos os parâmetros do modelo pelo método de máxima verossimilhança e discutimos medidas de influência global e local sob três esquemas de pertubação. Apresentamos também o resíduo quantílico. Concluímos o capítulo com uma aplicação do modelo proposto a dados reais.

\subsection{Introdução}

Como sabemos, os modelos de regressão lineares normais são geralmente aplicados para descrever dados simétricos por meio de funções lineares de parâmetros desconhecidos. No entanto, nem sempre os dados estão de acordo com o modelo normal devido à falta de simetria e/ou à presença de caudas pesadas/leves. Como uma alternativa, propomos um novo modelo de regressão com base no modelo Kumaraswamy normal.

O modelo proposto é baseado na suposição de que a resposta tem distribuição Kumaraswamy normal discutida no Capítulo 2.

\subsection{O modelo de regressão Kumaraswamy normal}

Com base na distribuição KwN, propomos um modelo de regressão linear do tipo localizaçãoescala que relaciona a variável resposta $y_{i}$ com o vetor de variáveis explicativas $\mathbf{x}_{i}=\left(x_{i 1}, \ldots, x_{i p}\right)^{\top}$, da seguinte forma

$$
y_{i}=\mathbf{x}_{i}^{\top} \boldsymbol{\beta}+\sigma z_{i}, i=1, \ldots, n
$$

em que os erros aleatórios $z_{i}$ têm distribuição dada em (2.5) independentes, $\boldsymbol{\beta}=\left(\beta_{1}, \ldots, \beta_{p}\right)^{\top}, \sigma>0$, $a>0$ e $b>0$ são parâmetros desconhecidos. Se, em (2.5), $a=b=1$, o modelo em (3.1) é o modelo de regressão normal.

A partir da equação (2.3), a função log-verossimilhança para o vetor de parâmetros $\boldsymbol{\eta}=$ 
$\left(a, b, \sigma, \boldsymbol{\beta}^{\top}\right)^{\top}$ pode ser expressa como

$$
\begin{aligned}
\ell(\boldsymbol{\eta})= & n\left[\log (a)+\log (b)-\log \left(\sigma^{2}\right)-\log (\sqrt{2 \pi})\right]+\sum_{i=1}^{n}\left\{(a-1) \log \left[\Phi\left(z_{i}\right)\right]\right. \\
& \left.+(b-1) \log \left[1-\Phi\left(z_{i}\right)^{a}\right]-\frac{z_{i}^{2}}{2}\right\}
\end{aligned}
$$

em que $z_{i}=\left(y_{i}-\mathbf{x}^{\top} \boldsymbol{\beta}\right) / \sigma$. O EMV $\hat{\boldsymbol{\eta}}$ de $\boldsymbol{\eta}$ pode ser obtido maximizando a função log-verossimilhança dada em (3.2). Por exemplo, podemos maximizar esta função usando a linguagem R (função optim), SAS (PROC NLMIXED) ou Ox (função MaxBFGS). Os componentes do vetor escore $U(\boldsymbol{\eta})$ são dados por

$$
\begin{aligned}
U_{a}(\boldsymbol{\eta}) & =\frac{n}{a}+\sum_{i=1}^{n}\left\{\log \left[\Phi\left(z_{i}\right)\right]-\frac{(b-1)\left[\Phi\left(z_{i}\right)\right]^{a} \log \left[\Phi\left(z_{i}\right)\right]}{1-\Phi\left(z_{i}\right)^{a}}\right\} \\
U_{b}(\boldsymbol{\eta}) & =\frac{n}{b}+\sum_{i=1}^{n} \log \left[1-\Phi\left(z_{i}\right)^{a}\right], \\
U_{\sigma}(\boldsymbol{\eta}) & =-\frac{2 n}{\sigma}+\frac{1}{\sigma} \sum_{i=1}^{n}\left\{z_{i}^{2}-z_{i} \phi\left(z_{i}\right)\left[\frac{(a-1)}{\Phi\left(z_{i}\right)}-\frac{a(b-1)\left[\Phi\left(z_{i}\right)\right]^{a-1}}{1-\Phi\left(z_{i}\right)^{a}}\right]\right\}
\end{aligned}
$$

e

$$
U_{\beta_{j}}(\boldsymbol{\eta})=\frac{1}{\sigma} \sum_{i=1}^{n} z_{i} x_{i j}-\frac{1}{\sigma} \sum_{i=1}^{n} \phi\left(z_{i}\right) x_{i j}\left\{\frac{(a-1)}{\Phi\left(z_{i}\right)}-\frac{a(b-1) \Phi\left(z_{i}\right)^{a-1}}{\left[1-\Phi\left(z_{i}\right)^{a}\right]}\right\},
$$

em que $j=1, \ldots, p$.

Sob condições gerais de regularidade, a distribuição assintótica de $\hat{\boldsymbol{\eta}}$ é

$$
\hat{\boldsymbol{\eta}}-\boldsymbol{\eta} \sim N_{p+3}\left(0, K(\boldsymbol{\eta})^{-1}\right),
$$

em que $K(\boldsymbol{\eta})^{-1}$ é a inversa da matriz de informação esperada. A matriz $K(\boldsymbol{\eta})^{-1}$ pode ser aproximada pela inversa da matriz de informação observada $J(\boldsymbol{\eta})$ dada por

$$
J(\boldsymbol{\eta})=\left[\begin{array}{cccc}
J_{a a} & J_{a b} & J_{a \sigma} & J_{a \beta_{1}} \ldots J_{a \beta_{p}} \\
J_{b a} & J_{b b} & J_{b \sigma} & J_{b \beta_{1}} \ldots J_{b \beta_{p}} \\
J_{\sigma a} & J_{\sigma b} & J_{\sigma \sigma} & J_{\sigma \beta_{1}} \ldots J_{\sigma \beta_{p}} \\
J_{\beta_{1} a} & J_{\beta_{1} b} & J_{\beta_{1} \sigma} & J_{\beta_{1} \beta_{1}} \ldots J_{\beta_{1} \beta_{p}} \\
\vdots & \vdots & \vdots & \vdots \\
J_{\beta_{p} a} & J_{\beta_{p} b} & J_{\beta_{p} \sigma} & J_{\beta_{p} \beta_{1}} \ldots J_{\beta_{p} \beta_{p}}
\end{array}\right]
$$

em que os elementos da matriz $J(\boldsymbol{\eta})$ são dados no Apêndice B.

Portanto, um intervalo de confiança assintótico $100(1-\alpha) \%$ para cada parâmetro $\eta_{i}$ é dado por

$$
I C A_{i}=\left(\hat{\eta}_{i}-z_{\alpha / 2} \sqrt{-\widehat{J}^{i, i}}, \hat{\eta}_{i}+z_{\alpha / 2} \sqrt{-\widehat{J}^{i, i}}\right)
$$

em que $-\widehat{J}^{i, i}$ é o $i$-ésimo elemento da diagonal da matriz $[J(\boldsymbol{\eta})]^{-1}$, avaliado em $\hat{\boldsymbol{\eta}}$ e $z_{\alpha / 2}$ é o quantil 
$\alpha / 2$ da distribuição normal padrão.

\subsection{Análise de diagnóstico}

A fim de avaliar a sensibilidade dos EMVs, a influência global e a influência local sob três esquemas de perturbação são agora discutidas.

\subsubsection{Influência global}

A primeira ferramenta para realizar a análise de sensibilidade é a influência global a partir da delação de casos (ver Cook , 1977). A delação de casos é uma abordagem comum para estudar o efeito de excluir a $i$-ésima observação do conjunto de dados. A deleção de casos para o modelo (3.1) é dada por

$$
y_{l}=\mathbf{x}_{l}^{\top} \boldsymbol{\beta}+\sigma z_{l}, l=1, \ldots, n, l \neq i
$$

A seguir, uma quantidade com o subíndice " $(i)$ " significa a quantidade original com a $i$-ésima observação excluída. $l_{(i)}(\boldsymbol{\eta})$ é a função de log-verossimilhança para o modelo (3.3) e seja $\hat{\boldsymbol{\eta}}_{(i)}=$ $\left(\hat{a}_{(i)}, \hat{b}_{(i)}, \hat{\sigma}_{(i)}, \hat{\boldsymbol{\beta}}_{(i)}^{\top}\right)^{\top}$ a estimativa correspondente de $\boldsymbol{\eta}$. A idéia básica para avaliar a influência da $i$-ésima observação no EMV $\hat{\boldsymbol{\eta}}=\left(\hat{a}, \hat{b}, \hat{\sigma}, \hat{\boldsymbol{\beta}}^{\top}\right)^{\top}$ é comparar a diferença entre $\hat{\boldsymbol{\eta}}_{(i)}$ e $\hat{\boldsymbol{\eta}}$. Se a exclusão de uma observação influir seriamente nas estimativas, deve ser dada mais atenção a essa observação. Portanto, se $\hat{\boldsymbol{\eta}}_{(i)}$ estiver longe de $\hat{\boldsymbol{\eta}}$, então a $i$-ésima observação pode ser considerada como influente. A primeira medida de influência global é conhecida como distância de Cook generalizada sendo expressa por

$$
G D_{i}(\hat{\boldsymbol{\eta}})=\left(\hat{\boldsymbol{\eta}}-\hat{\boldsymbol{\eta}}_{(i)}\right)^{\top}\{-\hat{\mathbf{J}}(\boldsymbol{\eta})\}\left(\hat{\boldsymbol{\eta}}-\hat{\boldsymbol{\eta}}_{(i)}\right),
$$

em que $\hat{\mathbf{J}}(\boldsymbol{\eta})$ é a matriz $\mathbf{J}(\boldsymbol{\eta})$ avaliada em $\hat{\boldsymbol{\eta}}$.

Outra alternativa é avaliar os valores de $G D_{i}(\hat{\boldsymbol{\beta}})$ e de $G D_{i}(\hat{a}, \hat{b}, \hat{\sigma})$ que revelam o impacto da $i$-ésima observação nas estimativas de $\boldsymbol{\beta}$ e de $(a, b, \sigma)^{\top}$, respectivamente. Outra medida bem conhecida da diferença entre $\hat{\boldsymbol{\eta}}_{(i)}$ e $\hat{\boldsymbol{\eta}}$ é o afastamento da verossimilhança, dado por

$$
L D_{i}(\hat{\boldsymbol{\eta}})=2\left\{l(\hat{\boldsymbol{\eta}})-l\left(\hat{\boldsymbol{\eta}}_{(i)}\right)\right\}
$$

Além disso, também podemos calcular $\hat{\beta}_{j}-\hat{\beta}_{j(i)}(j=1, \ldots, p)$ para detectar a diferença entre $\hat{\beta}$ e $\hat{\beta}_{(i)}$.

Podem ser construídos gráficos das medidas $G D_{i}$ e $L D_{i}$ versus a ordem das observações, o que torna possível a identificação de potenciais observações influentes.

\subsubsection{Influência local}

Outra abordagem defendida por Cook (1986) é ponderar as observações em vez de removêlas. Uma medida sugerida por Cook definida como $L D(\boldsymbol{\omega})=2\left\{\ell(\hat{\boldsymbol{\eta}})-\ell\left(\hat{\boldsymbol{\eta}}_{\boldsymbol{\omega}}\right)\right\}$ é chamada de afastamento da verossimilhança, em que $\hat{\boldsymbol{\eta}}_{\boldsymbol{\omega}}$ denota o estimador de máxima verossimilhança sob o modelo pertubado e $\boldsymbol{\omega}=\left(\omega_{1}, \ldots, \omega_{n}\right)^{\top}$ é o vetor de pertubação aplicado ao modelo. Dessa forma, se a distância entre $\hat{\boldsymbol{\eta}}$ e $\hat{\boldsymbol{\eta}}_{\boldsymbol{\omega}}$ permanecer "pequena" quando $\boldsymbol{\omega}$ varia, temos a indicação de que o modelo ajustado é estável no que diz respeito ao esquema de pertubação utilizado. A curvatura normal para $\boldsymbol{\eta}$ na direção $\mathbf{d}$ (ver Cook, 1986) é dada por $C_{\mathbf{d}}(\boldsymbol{\eta})=2\left|\mathbf{d}^{\top} \boldsymbol{\Delta}^{\top}[\mathbf{J}(\boldsymbol{\eta})]^{-1} \boldsymbol{\Delta} \mathbf{d}\right|$, em que 
$\boldsymbol{\Delta}$ é uma matriz $(p+3) \times n$ e depende do esquema de pertubação usado, cujos elementos são $\Delta_{j i}=\left(\partial^{2} \ell(\boldsymbol{\eta} \mid \boldsymbol{\omega}) / \partial \eta_{j} \partial \omega_{i}\right), i=1, \ldots, p+3$ e $j=1, \ldots, n$, com todas as quantidades sendo avaliadas em $\boldsymbol{\omega}=\boldsymbol{\omega}_{0}$ e $\boldsymbol{\eta}=\hat{\boldsymbol{\eta}}$, em que $\boldsymbol{\omega}_{0}$ é o vetor de não pertubação do modelo. Assim, $C_{\mathbf{d}_{\text {max }}}$ é o maior autovalor da matriz $\mathbf{B}=-\boldsymbol{\Delta}^{\top}[\mathbf{J}(\boldsymbol{\eta})]^{-1} \boldsymbol{\Delta}$, e $d_{\max }$ o corresponde autovetor.

Para algumas formas de perturbação, apresentadas a seguir, calculou-se a matriz

$$
\boldsymbol{\Delta}=\left(\boldsymbol{\Delta}_{j i}\right)_{(p+3) \times n}=\left(\frac{\partial^{2} \ell(\boldsymbol{\eta} \mid \boldsymbol{\omega})}{\partial \eta_{j} \partial \omega_{i}}\right), i=1, \ldots, p+3 \quad \text { e } \quad j=1, \ldots, n .
$$

Para o modelo em (3.1), temos

\section{- Ponderação de casos}

Nesse esquema o objetivo é avaliar se as contribuições das observações com diferentes pesos afetarão as EMV de $\boldsymbol{\eta}$. Para o nosso modelo, a função de log-verossimilhança perturbada é dada por

$$
\begin{aligned}
\ell(\boldsymbol{\eta} \mid \boldsymbol{\omega})= & n\left[\log (a)+\log (b)-\log \left(\sigma^{2}\right)-\log (\sqrt{2 \pi})\right] \sum_{i=1}^{n} \omega_{i}+\sum_{i=1}^{n} \omega_{i}\left\{(a-1) \log \left[\Phi\left(z_{i}\right)\right]\right. \\
& \left.+(b-1) \log \left[1-\Phi\left(z_{i}\right)^{a}\right]-\frac{z_{i}^{2}}{2}\right\}
\end{aligned}
$$

em que $0 \leq \omega_{i} \leq 1$ e $\boldsymbol{\omega}_{0}=(1, \ldots, 1)^{\top}$ é o vetor de não pertubação. Os elementos da matriz $\boldsymbol{\Delta}=\left(\boldsymbol{\Delta}_{a}^{\top}, \boldsymbol{\Delta}_{b}^{\top}, \boldsymbol{\Delta}_{\sigma}^{\top}, \boldsymbol{\Delta}_{\beta}^{\top}\right)$ são obtidos numericamente.

\section{- Perturbação na variável resposta}

O interesse nesse esquema é detectar a sensibilidade do modelo quando a variável resposta é submetida a uma perturbação aditiva dada por $Y_{i}=y_{i}+\omega_{i} S_{y}$, em que $S_{y}$ é um fator de escala que pode ser a estimativa do desvio padrão da resposta observada $Y$ e $\omega_{i} \in \mathbb{R}$. A função logverossimilhança perturbada pode ser expressa como

$$
\begin{aligned}
\ell(\boldsymbol{\eta} \mid \boldsymbol{\omega})= & n\left[\log (a)+\log (b)-\log \left(\sigma^{2}\right)-\log (\sqrt{2 \pi})\right]+\sum_{i=1}^{n}\left\{(a-1) \log \left[\Phi\left(z_{i}^{*}\right)\right]\right. \\
& \left.+(b-1) \log \left[1-\Phi\left(z_{i}^{*}\right)^{a}\right]-\frac{z_{i}^{* 2}}{2}\right\}
\end{aligned}
$$

em que $z_{i}^{*}=\left[\left(y_{i}+\omega_{i} S_{y}\right)-\mathbf{x}_{i}^{\top} \boldsymbol{\beta}\right] / \sigma$ e $\boldsymbol{\omega}_{0}=(0, \ldots, 0)^{\top}$ é o vetor de não perturbação. Os elementos da matriz $\boldsymbol{\Delta}=\left(\boldsymbol{\Delta}_{a}^{\top}, \boldsymbol{\Delta}_{b}^{\top}, \boldsymbol{\Delta}_{\sigma}^{\top}, \boldsymbol{\Delta}_{\boldsymbol{\beta}}^{\top}\right)$ são obtidos numericamente.

\section{- Perturbação em uma variável explicativa}

O objetivo nesse tipo de pertubação é avaliar a sensibilidade do modelo sob pequenas pertubações em uma variável explicativa. Considere agora uma perturbação aditiva em uma determinada variável explicativa contínua, digamos $X_{q}$, definida por $x_{i q \omega}=x_{i q}+\omega_{i} S_{q}$, em que $S_{q}$ é um fator de escala e $\omega_{i} \in \mathbb{R}$. A função log-verossimilhança perturbada tem a seguinte forma

$$
\begin{aligned}
\ell(\boldsymbol{\eta} \mid \boldsymbol{\omega})= & n\left[\log (a)+\log (b)-\log \left(\sigma^{2}\right)-\log (\sqrt{2 \pi})\right]+\sum_{i=1}^{n}\left\{(a-1) \log \left[\Phi\left(z_{i}^{* *}\right)\right]\right. \\
& \left.+(b-1) \log \left[1-\Phi\left(z_{i}^{* *}\right)^{a}\right]-\frac{z_{i}^{* * 2}}{2}\right\}
\end{aligned}
$$


em que $z_{i}^{* *}=\left(y_{i}-\mathbf{x}_{i}^{* T} \boldsymbol{\beta}\right) / \sigma, \mathbf{x}_{i}^{* T} \beta=\beta_{1}+\beta_{2} x_{i 2}+\ldots+\beta_{q}\left(x_{i q}+\omega_{i} S_{q}\right)+\cdots+\beta_{p} x_{i p}$ e $\boldsymbol{\omega}_{0}=$ $(0, \ldots, 0)^{\top}$ é o vetor de não pertubação. Os elementos da matriz $\boldsymbol{\Delta}=\left(\boldsymbol{\Delta}_{a}^{\top}, \boldsymbol{\Delta}_{b}^{\top}, \boldsymbol{\Delta}_{\sigma}^{\top}, \boldsymbol{\Delta}_{\boldsymbol{\beta}}^{\top}\right)$ são obtidos numericamente.

\subsection{Análise de resíduos}

A fim de estudar as suposições dos erros, bem como a presença de outliers, consideramos o resíduo quantílico.

O resíduo quantílico normalizado foi introduzido por Dunn e Smyth (1996) e é utilizado para avaliar a adequabilidade de ajustes de modelos de regressão. Neste trabalho, estes resíduos foram utilizados para verificar a qualidade do ajuste do modelo de regressão KwN. Como $F(x)$ em (2.3) é contínua, então pelo Teorema da Transformação Integral, é também uma variável aleatória uniformemente distribuída no intervalo $(0,1)$. Nesse caso, os resíduos quantílicos normalizados são definidos como

$$
r_{q, i}=\Phi^{-1}\left[F\left(y_{i} ; \hat{\mu}, \hat{a}, \hat{b}, \hat{\sigma}\right)\right]
$$

em que $\Phi\left(\right.$.) denota a função de distribuição acumulada da distribuição normal padrão, $\hat{\mu}=\mathbf{x}_{i}^{\top} \hat{\boldsymbol{\beta}}$ e $\hat{a}, \hat{b}$ e $\hat{\sigma}$ denotam os estimadores de máxima verossimilhança de $a, b$ e $\sigma$, respectivamente. Segundo os autores, se os parâmetros do modelo são consistentemente estimados, então $r_{q, i}$ converge para uma distribuição normal padrão. Dessa forma, espera-se que os valores desses resíduos em um gráfico QQ-plot estejam próximos de uma reta.

\subsection{Aplicação}

Nesta seção utilizamos um conjunto de dados reais para ilustrar o desempenho do modelo de regressão linear Kumaraswamy normal.

\subsubsection{Falhas da fotocopiadora}

Os dados analisados referem-se ao tempo entre falhas de uma fotocopiadora nos seus primeiros $4 \frac{1}{2}$ anos de funcionamento, descritos em Murthy, Xie, e Jiang (2004, página 314). Os dados são formados por 41 valores durante este período.

O conjunto de dados foi analisado por meio de quatro modelos de regressão, os quais podem ser expressos da seguinte forma:

$$
y_{i}=\beta_{0}+\beta_{1} x_{i}+\sigma z_{i}, \quad i=1, \ldots, 41
$$

em que

- $y_{i}$ : tempo entre a $(i-1)$-ésima falha e a $i$-ésima falha (em dias), $i=2, \ldots, 41$, sendo $y_{1}$ o tempo (em dias) até a primeira falha.

- $x_{i}$ : número de cópias entre a $(i-1)$-ésima falha e a $i$-ésima falha, $i=2, \ldots, 41$, sendo $x_{1}$ o número de cópias até a primeira falha. 
- $z_{i}$ tem distribuição KwN padrão, Lehmann tipo II normal (LTII-N), Exp-N e normal.

- $y_{i}^{\prime} s$ são independentes, $i=1,2, \ldots, 41$.

A Tabela 3.1 apresenta, para cada modelo de regressão, as estimativas dos parâmetros com os respectivos erros padrãos (entre parênteses) e os valores numéricos dos critérios de informação AIC, CAIC e BIC. Os parâmetros foram estimados usando a linguagem R. O modelo de regressão KwN superou os modelos de regressão LTII-N, Exp-N e normal, apresentando os menores valores para os referidos critérios de informação, o que indica que o modelo de regressão $\mathrm{KwN}$ é o que melhor se ajusta aos dados.

Na Figura 3.1, apresentamos o gráfico dos resíduos quantílicos versus os valores ajustados. Notamos que não há nenhum resíduo alto (fora do intervalo $[-3,3]$ ).

Na Figura 3.2 temos o gráfico normal de probabilidade para os resíduos quantílicos com envelope simulado, o qual foi usado para verificar a qualidade do ajuste do modelo de regressão KwN. Através do mesmo notamos que o modelo de regressão KwN se encontra bem ajustado aos dados sob análise, pois todos os pontos estão dentro do envelope gerado. Fizemos esse mesmo gráfico para os demais modelos (LTII-N, Exp-N e normal), mas os mesmos não ficaram bem ajustados.

Apresentamos na Figura 3.3, o gráfico da distância de Cook generalizada. Como podemos ver as observações \#2, \#6 e \#41 foram detectadas como sendo possíveis observações influentes. Com o intuito de analisar o impacto dessas observações nas estimativas dos parâmetros, realizamos uma análise confirmatória reajustando o modelo sem as observações consideradas influentes. Depois calculamos a mudança relativa em porcentagem das estimativas para os parâmetros do modelo, definida por

$$
M R_{\eta}(i)=\left|\frac{\hat{\eta}-\hat{\eta}_{(i)}}{\hat{\eta}}\right| \times 100 \%,
$$

em que $\hat{\eta}$ denota a estimativa do parâmetro $\eta$ considerando todas as observações e $\hat{\eta}_{(i)}$ denota a estimativa do parâmetro $\eta$ após a retirada da observação $i$. As mudanças relativas para as observações \#2, \#6 e \#41, individualmente e para as observações \#2 e \#41 conjuntamente estão apresentadas na Tabela 3.2.

Na Figura 3.4, construímos o gráfico de influência local para o esquema de perturbação ponderação de casos, por ser o mais comum, objetivando identificar observações influentes. Note que as observações \#6 e \#13 se apresentam como possíveis casos influentes. A observação \#6 já havia sido detectada como possível observação influente por meio do gráfico da distância de Cook generalizada, mas como mostra a Tabela 3.2 a mesma não apresentou mudança relativa substancial individualmente. A mudança relativa para a observação \#13 está disponível na Tabela 3.3, assim como a mudança relativa para as observações \#2, \#13 e \#41, conjuntamente.

$\mathrm{Na}$ Tabela 3.4 apresentamos as estimativas dos parâmetros do modelo de regressão KwN retirando essas observações. Notamos que não houve mudança inferencial com a retirada das observações \#2, \#6, \#13 e \#41. Portanto, nenhuma das observações deve ser descartada. 
Tabela 3.1: Estimativas de máxima verossimilhança e respectivos erros padrão dos parâmetros para os modelos de regressão $\mathrm{KwN}$, LTII-N, Exp-N e normal ajustados aos dados do tempo entre falhas da fotocopiadora e valores dos critérios de informação

\begin{tabular}{c|ccccc|ccc}
\hline \hline \multirow{2}{*}{ Distribuição } & \multicolumn{5}{|c|}{ Parâmetros } & \multicolumn{3}{c}{ Critérios } \\
\cline { 2 - 8 } & $a$ & $b$ & $\sigma$ & $\beta_{0}$ & $\beta_{1}$ & AIC & CAIC & BIC \\
\hline \multirow{2}{*}{ KwN } & 0,5207 & 0,1514 & 7,7608 & 6,0462 & 0,7276 & 533,2 & 534,9 & 541,8 \\
& $(0,0047)$ & $(0,0236)$ & $(0,0024)$ & $(0,0222)$ & $(0,0023)$ & & & \\
LTII-N & 1 & 0,1386 & 7,3063 & 10,5480 & 0,5551 & 536,9 & 538,0 & 543,8 \\
& - & $(0,0231)$ & $(0,4368)$ & $(1,0419)$ & $(0,0026)$ & & & \\
Exp-N & 0,5914 & 1 & 12,0773 & 19,3516 & 0,9363 & 580,6 & 581,7 & 587,4 \\
& $(-)$ & - & $(-)$ & $(-)$ & $(0,0946)$ & & & \\
Normal & 1 & 1 & 18,87 & 18,4167 & 0,8147 & 602,1 & 602,7 & 607,2 \\
& - & - & $(0,2413)$ & $(4,4202)$ & $(0,1228)$ & & & \\
\hline \hline
\end{tabular}

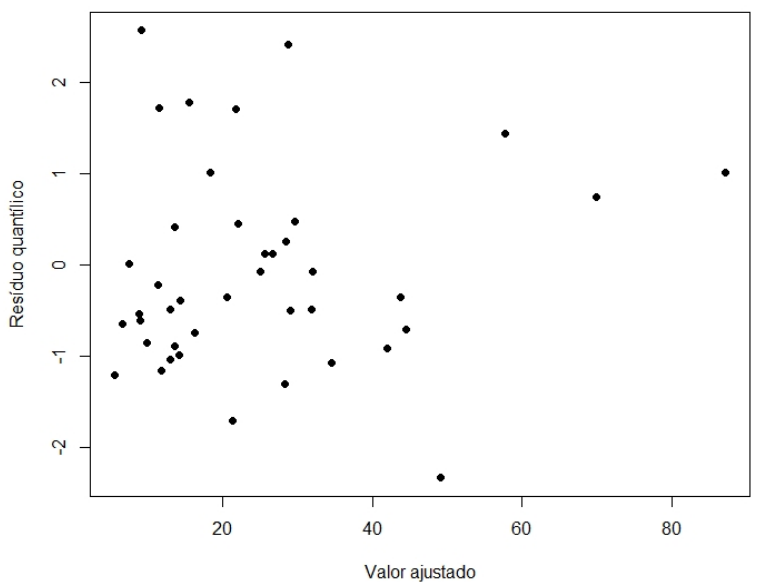

Figura 3.1: Gráfico dos resíduos quantílicos versus valores ajustados para o modelo de regressão $K w N$.

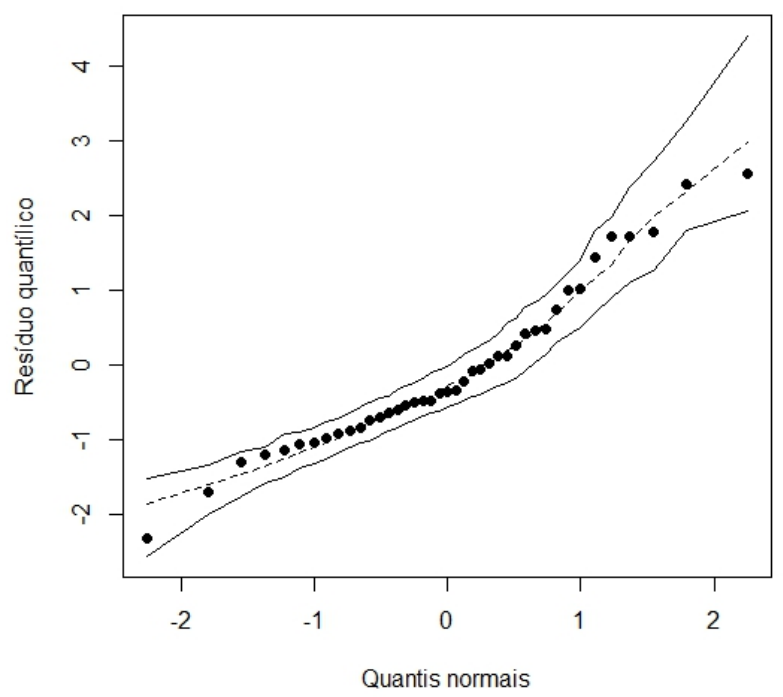

Figura 3.2: Gráfico de envelope simulado dos resíduos quantílicos para o modelo de regressão KwN. 


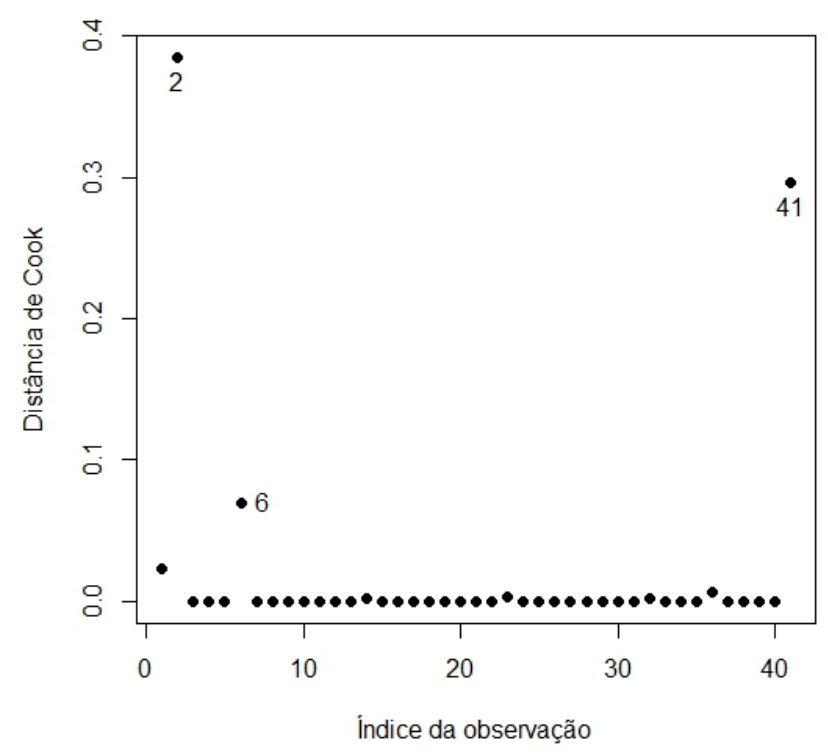

Figura 3.3: Gráfico da distância de Cook generalizada para o modelo de regressão KwN.

Tabela 3.2: Mudanças relativas

\begin{tabular}{c|ccccc}
\hline \hline \multirow{2}{*}{ Observações } & \multicolumn{5}{|c}{ Parâmetros } \\
\cline { 2 - 6 } & $a$ & $b$ & $\sigma$ & $\beta_{0}$ & $\beta_{1}$ \\
\hline$\# 2$ & $37,99 \%$ & $6,55 \%$ & $0,96 \%$ & $5,12 \%$ & $5,98 \%$ \\
$\# 6$ & $3,32 \%$ & $11,15 \%$ & $4,06 \%$ & $2,17 \%$ & $2,10 \%$ \\
$\# 41$ & $42,93 \%$ & $4,04 \%$ & $0,99 \%$ & $4,50 \%$ & $4,50 \%$ \\
$\# 2$ e \#41 & $37,99 \%$ & $0,51 \%$ & $3,60 \%$ & $15,98 \%$ & $5,39 \%$ \\
\hline \hline
\end{tabular}

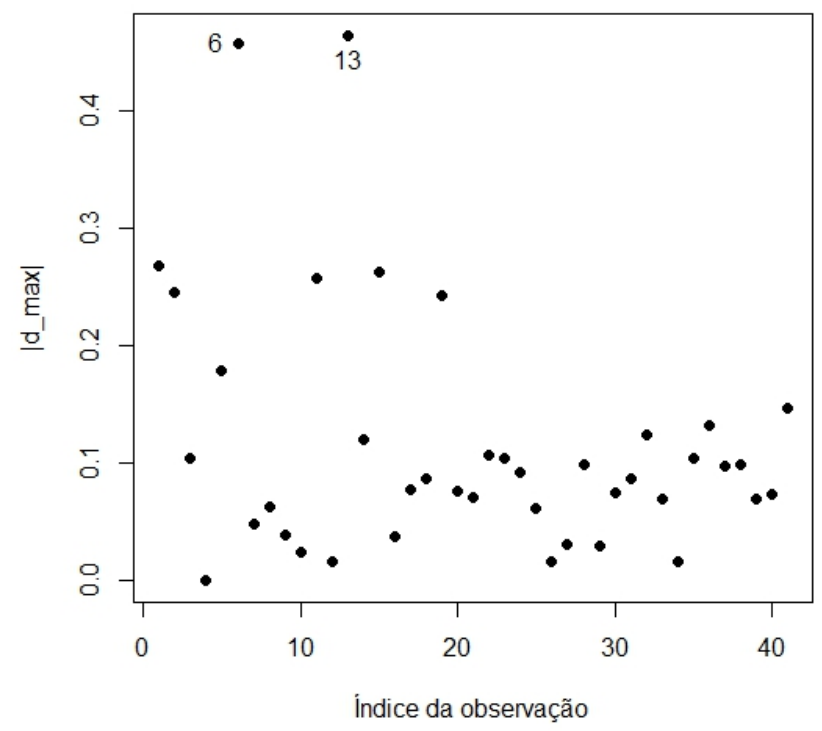

Figura 3.4: Gráfico de $\left|d_{\max }\right|$ contra indice da observação considerando o esquema de perturbação da ponderação de casos. 
Tabela 3.3: Mudanças relativas

\begin{tabular}{c|ccccc}
\hline \hline \multirow{2}{*}{ Observações } & \multicolumn{5}{|c}{ Parâmetros } \\
\cline { 2 - 6 } & $\mathrm{a}$ & $\mathrm{b}$ & $\sigma$ & $\beta_{0}$ & $\beta_{1}$ \\
\hline$\# 13$ & $22,14 \%$ & $13,28 \%$ & $0,01 \%$ & $0,05 \%$ & $0,05 \%$ \\
$\# 2, \# 13$ e \#41 & $29,02 \%$ & $10,59 \%$ & $3,38 \%$ & $29,43 \%$ & $3,14 \%$ \\
\hline \hline
\end{tabular}

Tabela 3.4: Estimativas de máxima verossimilhança e respectivos erros padrão dos parâmetros para os modelos de regressão $\mathrm{KwN}$, ajustados aos dados do tempo entre falhas da fotocopiadora sem as observações.

\begin{tabular}{c|ccccc}
\hline & $\mathrm{a}$ & $\mathrm{b}$ & $\sigma$ & $\beta_{0}$ & $\beta_{1}$ \\
\hline$\# 2$ & 0,6997 & 0,1556 & 7,6419 & 5,5366 & 0,6834 \\
& $(0,0046)$ & $(0,0235)$ & $(0,0024)$ & $(0,0026)$ & $(0,0023)$ \\
$\# 6$ & 0,5024 & 0,1624 & 7,4028 & 5,6621 & 0,7421 \\
$\# 13$ & $(0,0041)$ & $(0,0231)$ & $(0,0023)$ & $(0,0022)$ & $(0,0021)$ \\
& 0,6193 & 0,1655 & 7,7163 & 5,4215 & 0,7271 \\
$\# 41$ & $(0,0042)$ & $(0,0232)$ & $(0,0021)$ & $(0,0018)$ & $(0,0022)$ \\
& 0,7247 & 0,1402 & 7,6393 & 5,6621 & 0,6941 \\
$\# 2$ e \#41 & $(0,0045)$ & $(0,0218)$ & $(0,0022)$ & $(0,0220)$ & $(0,0021)$ \\
$\# 2, \# 13$ e \#41 & 0,6997 & 0,1453 & 7,4383 & 6,2844 & 0,6876 \\
& $(0,0040)$ & $(0,0221)$ & $(0,0021)$ & $(0,0210)$ & $(0,0018)$ \\
& 0,6542 & 0,1615 & 7,4552 & 7,0131 & 0,7039 \\
& $(0,0039)$ & $(0,0216)$ & $(0,0020)$ & $(0,0209)$ & $(0,0020$ \\
\hline \hline
\end{tabular}




\title{
Capítulo 4
}

\section{Modelo de regressão Kumaraswamy normal com presença de censura}

\begin{abstract}
Neste capitulo propomos o modelo de regressão KwN para dados censurados. Discutimos a estimação dos parâmetros do modelo e apresentamos medidas de influência local sob três esquemas de pertubação. Apresentamos também os resíduos martingal e deviance modificado. Aplicamos o novo modelo a dados reais.
\end{abstract}

\subsection{Introdução}

Estudos que envolvem uma resposta temporal, geralmente são de longa duração, e na maioria dos casos, existem unidades experimentais que não chegam ao evento de interesse, ou seja, há ocorrência de observações incompletas ou parciais, denominadas censuras. A importância da análise de sobrevivência reside na possibilidade de inserir na análise estatística a informação contida nos dados censurados.

Várias distribuições têm sido utilizadas na análise dados de sobrevivência nos últimos anos. Por exemplo, Leiva et al. (2007) fizeram um estudo de diagnóstico em um modelo de regressão com censura log-Birnbaum-Saunders. Carrasco et al. (2008) propuseram o modelo de regressão baseado em uma modificação da distribuição Weibull. Ortega et al. (2011) propuseram o modelo de regressão beta-Weibull.

\subsection{Modelo de regressão Kumaraswamy normal com censura}

Nesta seção, propomos o modelo de regressão linear KwN do tipo locação-escala com presença de censura aleatória dado por

$$
y_{i}=\mathbf{x}_{i}^{\top} \boldsymbol{\beta}+\sigma z_{i}, i=1, \ldots, n,
$$

em que $y_{i}=\min \left\{\log \left(t_{i}\right), \log \left(c_{i}\right)\right\}$, sendo $t_{i}$ o tempo de falha e $c_{i}$ o tempo de censura, $\mathbf{x}_{i}=$ $\left(x_{i 1}, \ldots, x_{i p}\right)^{\top}$ o vetor de variáveis explicativas, $z_{i}$ o erro aleatório com distribuição dada em (2.5) independentes, $\boldsymbol{\beta}=\left(\beta_{1}, \ldots, \beta_{p}\right)^{\top}, \sigma>0, a>0$ e $b>0$ parâmetros desconhecidos. 
Usando o modelo em (4.1), a função de sobrevivência de $Y_{i} \mid \mathbf{x}_{i}$ é dada por

$$
S\left(y_{i} ; \boldsymbol{\eta}\right)=\left[1-\Phi\left(\frac{y_{i}-\mathbf{x}_{i}^{\top} \boldsymbol{\beta}}{\sigma}\right)^{a}\right]^{b}
$$

em que $\boldsymbol{\eta}=\left(a, b, \sigma, \boldsymbol{\beta}^{\top}\right)$.

Assumindo censura não informativa (não carrega informações sobre os parâmetros) e definindo os conjuntos $\mathrm{F}$, de indivíduos que falharam, e C, de indivíduos censurados, a função de verossimilhança, considerando os tempos de falha e de censura, pode ser expressa como

$$
\begin{aligned}
L(\boldsymbol{\eta}) & =\prod_{i \in F} f\left(y_{i} ; \eta\right) \prod_{i \in C} S\left(y_{i} ; \eta\right) \\
& =\prod_{i \in F}\left[\frac{a b}{\sigma} \phi\left(z_{i}\right) \Phi\left(z_{i}\right)^{a-1}\left\{1-\Phi\left(z_{i}\right)^{a}\right\}^{b-1}\right] \prod_{i \in C}\left\{\left[1-\Phi\left(z_{i}\right)^{a}\right]^{b}\right\}
\end{aligned}
$$

em que $z_{i}=\left(y_{i}-\mathbf{x}_{i}^{\top} \boldsymbol{\beta}\right) / \sigma$.

A função de log-verossimilhança é dado por

$$
\begin{aligned}
\ell(\boldsymbol{\eta})= & \sum_{i \in F}\left\{\log \left(\frac{a b}{\sigma^{2} \sqrt{2 \pi}}\right)+(a-1) \log \left[\Phi\left(z_{i}\right)\right]+(b-1) \log \left[1-\Phi\left(z_{i}\right)^{a}\right]\right. \\
& \left.-\frac{z_{i}^{2}}{2}\right\}+\sum_{i \in C} \log \left\{\left[1-\Phi\left(z_{i}\right)^{a}\right]^{b}\right\} .
\end{aligned}
$$

O estimador de máxima verossimilhança (EMV) $\hat{\boldsymbol{\eta}}$ de $\boldsymbol{\eta}$ pode ser obtido por meio da maximização da função de log-verossimilhança (4.3). Podemos maximizar a função em (4.3) utilizando o procedimento NLMixed do SAS, MaxBFGS do OX ou a linguagem R (função optim), por exemplo.

Os componentes do vetor escore $U(\boldsymbol{\eta})$ são dados por

$$
\begin{aligned}
U_{a}(\boldsymbol{\eta})= & \sum_{i \in F}\left\{\frac{1}{a}+\log \left[\Phi\left(z_{i}\right)\right]-\frac{(b-1)\left[\Phi\left(z_{i}\right)\right]^{a} \log \left[\Phi\left(z_{i}\right)\right]}{1-\Phi\left(z_{i}\right)^{a}}\right\}-b \sum_{i \in C} \frac{\Phi\left(z_{i}\right)^{a} \log \left[\Phi\left(z_{i}\right)\right]}{1-\Phi\left(z_{i}\right)^{a}}, \\
U_{b}(\boldsymbol{\eta})= & \sum_{i \in F}\left\{\frac{1}{b}+\log \left[1-\Phi\left(z_{i}\right)^{a}\right]\right\}+\sum_{i \in C} \log \left[1-\Phi\left(z_{i}\right)^{a}\right], \\
U_{\sigma}(\boldsymbol{\eta})= & \frac{1}{\sigma} \sum_{i \in F}\left\{z_{i}^{2}-2-z_{i} \phi\left(z_{i}\right)\left[\frac{(a-1)}{\Phi\left(z_{i}\right)}-\frac{a(b-1)\left[\Phi\left(z_{i}\right)\right]^{a-1}}{1-\Phi\left(z_{i}\right)^{a}}\right]\right\} \\
& +\sum_{i \in C} \frac{a b \Phi\left(z_{i}\right)^{a-1} \phi\left(z_{i}\right) z_{i}}{\sigma\left[1-\Phi\left(z_{i}\right)^{a}\right]}
\end{aligned}
$$

e

$$
\begin{aligned}
U_{\beta_{j}}(\boldsymbol{\eta})= & \frac{1}{\sigma} \sum_{i \in F}\left\{z_{i} x_{i j}-\phi\left(z_{i}\right) x_{i j}\left[\frac{(a-1)}{\Phi\left(z_{i}\right)}-\frac{a(b-1) \Phi\left(z_{i}\right)^{a-1}}{\left[1-\Phi\left(z_{i}\right)^{a}\right]}\right]\right\} \\
& +\sum_{i \in C} \frac{a b \Phi\left(z_{i}\right)^{a-1} \phi\left(z_{i}\right) x_{i j}}{\sigma\left[1-\Phi\left(z_{i}\right)^{a}\right]}
\end{aligned}
$$

em que $j=1, \ldots, p$. Do modelo ajustado em (4.1), a função de sobrevivência para $y_{i}$ pode ser 
estimada por

$$
\hat{S}\left(y_{i}, \boldsymbol{\eta}\right)=\left[1-\Phi\left(\frac{y_{i}-\mathbf{x}_{i}^{\top} \hat{\boldsymbol{\beta}}}{\hat{\sigma}}\right)^{\hat{a}}\right]^{\hat{b}} .
$$

Sob condições gerais de regularidade, a distribuição assintótica de $\hat{\boldsymbol{\eta}}$ é

$$
\hat{\boldsymbol{\eta}}-\boldsymbol{\eta} \sim N_{(p+3)}\left(0, K(\boldsymbol{\eta})^{-1}\right),
$$

em que $K(\boldsymbol{\eta})^{-1}$ é a inversa da matriz de informação de Fisher. Como o cálculo da matriz $K(\boldsymbol{\eta})^{-1}$ é difícil, podemos usar como alternativa, a matriz de informação observada, denotada por $J(\boldsymbol{\eta})$.Neste caso, a matriz de informação observada é dada por

$$
J(\boldsymbol{\eta})=\left[\begin{array}{cccc}
J_{a a} & J_{a b} & J_{a \sigma} & J_{a \beta_{1}} \ldots J_{a \beta_{p}} \\
J_{b a} & J_{b b} & J_{b \sigma} & J_{b \beta_{1}} \ldots J_{b \beta_{p}} \\
J_{\sigma a} & J_{\sigma b} & J_{\sigma \sigma} & J_{\sigma \beta_{1}} \ldots J_{\sigma \beta_{p}} \\
J_{\beta_{1} a} & J_{\beta_{1} b} & J_{\beta_{1} \sigma} & J_{\beta_{1} \beta_{1}} \ldots J_{\beta_{1} \beta_{p}} \\
\vdots & \vdots & \vdots & \vdots \\
J_{\beta_{p} a} & J_{\beta_{p} b} & J_{\beta_{p} \sigma} & J_{\beta_{p} \beta_{1}} \ldots J_{\beta_{p} \beta_{p}}
\end{array}\right],
$$

em que os elementos da matriz $J(\boldsymbol{\eta})$ são dados no Apêndice C.

Portanto, um intervalo de confiança assintótico $100(1-\alpha) \%$ para cada parâmetro $\eta_{i}$ é dado por

$$
I C A_{i}=\left(\hat{\eta}_{i}-z_{\alpha / 2} \sqrt{-\widehat{J}^{i, i}}, \hat{\eta}_{i}+z_{\alpha / 2} \sqrt{-\widehat{J}^{i, i}}\right)
$$

em que $-\widehat{J}^{i, i} i$-ésimo elemento da diagonal da matriz $[J(\boldsymbol{\eta})]^{-1}$ avaliado em $\hat{\boldsymbol{\eta}}$ e $z_{\alpha / 2}$ é o quantil $\alpha / 2$ da distribuição normal padrão.

\subsection{Análise de diagnóstico}

Para avaliar a sensibilidade dos EMV utilizaremos a influência local sob três esquemas de perturbação.

\subsubsection{Influência local}

Nesta seção aplicaremos a técnica de influência local descrita na Seção 3.3.2.

Para alguns regimes de perturbação, apresentados a seguir, calculou-se a matriz

$$
\boldsymbol{\Delta}=\left(\boldsymbol{\Delta}_{j i}\right)_{(p+3) \times n}=\left(\frac{\partial^{2} \ell(\boldsymbol{\eta} \mid \boldsymbol{\omega})}{\partial \eta_{j} \partial \omega_{i}}\right), i=1, \ldots, p+3 \quad \text { e } \quad j=1, \ldots, n .
$$

Para o modelo em (4.1), temos

\section{- Ponderação de casos}

O objetivo nesse esquema é avaliar se as contribuições das observações com diferentes pesos afetarão as estimativas de máxima verossimilhança de $\eta$. Para o nosso modelo, a função de log- 
verossimilhança perturbada é dada por

$$
\begin{aligned}
\ell(\boldsymbol{\eta} \mid \boldsymbol{\omega})= & r\left[\log (a)+\log (b)-\log \left(\sigma^{2}\right)-\log (\sqrt{2 \pi})\right] \sum_{i \in F} \omega_{i}+\sum_{i \in F} \omega_{i}\left\{(a-1) \log \left[\Phi\left(z_{i}\right)\right]+\right. \\
& \left.+(b-1) \log \left[1-\Phi\left(z_{i}\right)^{a}\right]-\frac{z_{i}^{2}}{2}\right\}+\sum_{i \in C} \omega_{i} \log \left\{\left[1-\Phi\left(z_{i}\right)^{a}\right]^{b}\right\}
\end{aligned}
$$

em que $r$ é o número de falhas, $0 \leq \omega_{i} \leq 1$ e $\boldsymbol{\omega}_{0}=(1, \ldots, 1)^{\top}$. Os elementos da matriz $\boldsymbol{\Delta}=$ $\left(\boldsymbol{\Delta}_{a}^{\top}, \boldsymbol{\Delta}_{b}^{\top}, \boldsymbol{\Delta}_{\sigma}^{\top}, \boldsymbol{\Delta}_{\boldsymbol{\beta}}^{\top}\right)$ são obtidos numericamente.

\section{- Perturbação na variável resposta}

Neste esquema, o interesse é detectar a sensibilidade do modelo quando a variável resposta é submetida a uma perturbação aditiva dada por $Y_{i}=y_{i}+\omega_{i} S_{y}$, em que $S_{y}$ é um fator de escala que pode ser a estimativa do desvio padrão da resposta observada $Y$ e $\omega_{i} \in \Re$. A função de log-verossimilhança perturbada pode ser expressa como

$$
\begin{aligned}
\ell(\boldsymbol{\eta} \mid \boldsymbol{\omega})= & r\left[\log (a)+\log (b)-\log \left(\sigma^{2}\right)-\log (\sqrt{2 \pi})\right]+\sum_{i \in F}\left\{(a-1) \log \left[\Phi\left(z_{i}^{*}\right)\right]+\right. \\
& \left.+(b-1) \log \left[1-\Phi\left(z_{i}^{*}\right)^{a}\right]-\frac{z_{i}^{* 2}}{2}\right\}+\sum_{i \in C} \log \left\{\left[1-\Phi\left(z_{i}^{*}\right)^{a}\right]^{b}\right\}
\end{aligned}
$$

em que $z_{i}^{*}=\left[\left(y_{i}+\omega_{i} S_{y}\right)-\mathbf{x}_{i}^{\top} \boldsymbol{\beta}\right] / \sigma$ e $\boldsymbol{\omega}_{0}=(0, \ldots, 0)^{\top}$ é o vetor de não perturbação. Os elementos da matriz $\boldsymbol{\Delta}=\left(\boldsymbol{\Delta}_{a}^{\top}, \boldsymbol{\Delta}_{b}^{\top}, \boldsymbol{\Delta}_{\sigma}^{\top}, \boldsymbol{\Delta}_{\boldsymbol{\beta}}^{\top}\right)$ são obtidos numericamente.

\section{- Perturbação em uma variável explicativa}

Para verificar a sensibilidade do modelo (4.1), considere agora uma perturbação aditiva em uma determinada variável explicativa contínua, digamos $X_{q}$, definida por $x_{i q \omega}=x_{i q}+\omega_{i} S_{q}$, em que $S_{q}$ é um vetor de escala e $\omega_{i} \in \Re$. A função de log-verossimilhança perturbada tem a seguinte forma

$$
\begin{aligned}
\ell(\boldsymbol{\eta} \mid \boldsymbol{\omega})= & r\left[\log (a)+\log (b)-\log \left(\sigma^{2}\right)-\log (\sqrt{2 \pi})\right]+\sum_{i \in F}\left\{(a-1) \log \left[\Phi\left(z_{i}^{* *}\right)\right]+\right. \\
& \left.+(b-1) \log \left[1-\Phi\left(z_{i}^{* *}\right)^{a}\right]-\frac{z_{i}^{* * 2}}{2}\right\}+\sum_{i \in C} \log \left\{\left[1-\Phi\left(z_{i}^{* *}\right)^{a}\right]^{b}\right\},
\end{aligned}
$$

em que $z_{i}^{* *}=\left(y_{i}-\mathbf{x}_{i}^{* \top} \boldsymbol{\beta}\right) / \sigma, \mathbf{x}_{i}^{* \top} \boldsymbol{\beta}=\beta_{1}+\beta_{2} x_{i 2}+\cdots+\beta_{q}\left(x_{i q}+\omega_{i} S_{q}\right)+\cdots+\beta_{p} x_{i p}$ e $\boldsymbol{\omega}_{0}=$ $(0, \ldots, 0)^{\top}$ é o fator de não pertubação. Os elementos da matriz $\boldsymbol{\Delta}=\left(\boldsymbol{\Delta}_{a}^{\top}, \boldsymbol{\Delta}_{b}^{\top}, \boldsymbol{\Delta}_{\sigma}^{\top}, \boldsymbol{\Delta}_{\boldsymbol{\beta}}^{\top}\right)$ são obtidos numericamente.

\subsection{Análise de resíduos}

A fim de verificar as suposições dos erros, bem como a presença de outliers, consideramos dois tipos de resíduos: resíduo martingal e resíduo deviance modificado.

\section{- Resíduo martingal}


O resíduo martingal é muito utilizado em processos de contagem (Fleming e Harrington , 2011). O resíduo martingal é definido como $\hat{r}_{M_{i}}=\delta_{i}+\log \left[\hat{S}\left(y_{i} ; \boldsymbol{\eta}\right)\right]$, em que $\delta_{i}$ é a variável indicadora de censura, ou seja, $\delta_{i}=0\left(\delta_{i}=1\right)$ e $\hat{S}\left(y_{i} ; \boldsymbol{\eta}\right)$ denota a função de sobrevivência estimada expressa em (4.2). Portanto, o resíduo martingal para o modelo $\mathrm{KwN}$ assume a forma

$$
\hat{r}_{M_{i}}=\left\{\begin{array}{l}
1+\log \left\{\left[1-\Phi\left(z_{i}\right)^{\hat{a}}\right]^{\hat{b}}\right\}, \quad \text { se } \delta_{\mathrm{i}}=1, \\
\log \left\{\left[1-\Phi\left(z_{i}\right)^{\hat{a}}\right]^{\hat{b}}\right\}, \quad \text { se } \delta_{\mathrm{i}}=0,
\end{array}\right.
$$

em que $z_{i}=\left(y_{i}-\mathbf{x}_{i}^{\top} \hat{\boldsymbol{\beta}}\right) / \hat{\sigma}$.

Esse resíduo é assimétrico e assume valores no intervalo $(-\infty, 1]$.

\section{- Resíduo deviance modificado}

Esse resíduo foi introduzido por Therneau et al. (1990) visando tornar o resíduo martingal mais simétrico em torno de zero. O resíduo deviance modificado para um modelo de regressão paramétrico é dado por

$$
\hat{r}_{D_{i}}=\operatorname{sinal}\left(\hat{r}_{M_{i}}\right)\left\{-2\left[\hat{r}_{M_{i}}+\delta_{i} \log \left(\delta_{i}-\hat{r}_{M_{i}}\right)\right]\right\}^{1 / 2}
$$

em que $\hat{r}_{M_{i}}$ é o resíduo martingal e a função $\operatorname{sinal}()$ é uma função que conduz aos valores +1 se o argumento é positivo e -1 se o argumento é negativo. O resíduo deviance modificado para o nosso modelo é expresso por

$$
\hat{r}_{D_{i}}=\left\{\begin{array}{l}
\operatorname{sinal}\left(\hat{r}_{M_{i}}\right)\left\{-2\left[1+\log \left\{\left[1-\Phi\left(z_{i}\right)^{\hat{a}}\right]^{\hat{b}}\right\}+\log \left(-\log \left\{\left[1-\Phi\left(z_{i}\right)^{\hat{a}}\right]^{\hat{b}}\right\}\right)\right]\right\}^{1 / 2}, \text { se } \delta_{\mathrm{i}}=1, \\
\operatorname{sinal}\left(\hat{r}_{M_{i}}\right)\left\{-2\left(\log \left\{\left[1-\Phi\left(z_{i}\right)^{\hat{a}}\right]^{\hat{b}}\right\}\right)\right\}^{1 / 2}, \text { se } \delta_{\mathrm{i}}=0 .
\end{array}\right.
$$

O gráfico dos resíduos, martingal ou deviance modificado versus os valores ajustados forma uma ferramenta importante na análise residual, pois o mesmo fornece uma maneira de verificar a adequação do modelo e auxiliam na deteç̧ão de observações atípicas (outliers). Se o modelo for adequado, os gráficos devem apresentar um comportamento aleatório em torno de zero.

\subsection{Aplicação}

Nesta seção, utilizamos um conjunto de dados reais analisado por Kalbfleisch e Prentice (1980) para ilustrar a utilidade do modelo de regressão KwN com censura aleatória. Estimamos os parâmetros desse modelo por máxima verossimilhança usando o procedimento BFGS em linguagem R. O conjunto de dados é descrito em seguida.

\subsubsection{Dados de transplante de coração em Stanford}

Os dados reportam-se ao programa de transplante de coração de Stanford no período decorrido entre outubro de 1967 a abril de 1974 e referem-se a 103 pacientes cardíacos que se cadastraram no programa. Dentre os 103 pacientes, 69 receberam o transplante. O número de mortes registradas no final do estudo foi de 75 (45 com transplante e $30 \mathrm{sem}$ terem recebido transplante) e os 28 pacientes restantes contribuíram com tempos de sobrevida censurados. Disponibilizamos os dados no Apêndice D. 
O conjunto de dados é analisado sob a perspectiva de modelos de regressão com censura aleatória à direita, que podem ser expressos da seguinte forma

$$
y_{i}=\beta_{0}+\beta_{1} x_{i 1}+\beta_{2} x_{i 2}+\beta_{3} x_{i 3}+\sigma z_{i}, \quad i=1, \ldots, 103 .
$$

Consideramos as seguintes variáveis:

- $y_{i}$ : tempo de sobrevida (tempo desde a aceitação para o programa de transplante de coração até a morte, em dias) ou tempo de censura.

- $x_{i 1}$ : idade de aceitação (idade do indivíduo quando incluído no programa de transplante de coração, em anos),

- $x_{i 2}$ : cirurgia prévia $(1=\operatorname{sim}, 0=$ não),

- $x_{i 3}$ : transplante $(1=\operatorname{sim}, 0=$ não $)$.

As Tabelas 4.1 e 4.2 apresentam as estimativas de máxima verossimilhança e erros padrões (entre parênteses) dos parâmetros dos modelos de regressão e os valores dos critérios de informação AIC, BIC e CAIC para os modelos de regressão KwN, LTII-N, Exp-N e Normal. Observamos que o modelo de regressão $\mathrm{KwN}$ fornece valores mais baixos para os critérios AIC, BIC e CAIC quando comparado aos modelos de regressão LTII-N, Exp-N e normal. Assim, concluímos que o modelo de regressão KwN é o que melhor se adapta aos dados de transplante de coração.

Como podemos observar na Tabela 4.1, a estimativa para o parâmetro $\beta_{1}$ é negativa para o modelo KwN assim como para os demais modelos, exceto para o modelo Exp-N. Esse resultado faz sentido, pois $\beta_{1}$ é a idade de aceitação no programa e quanto maior for, menor será o tempo de vida.

A fim de detectar possíveis observações discrepantes no modelo de regressão KwN, construímos o gráfico dos resíduos deviance modificado versus valores ajustados, disponível na Figura 4.1. Não detectamos nenhuma tendência, apesar de ter ocorrido um valor de resíduo inferior a -3 .

O gráfico normal de probabilidade dos resíduos deviance modificado com envelope simulado é apresentado na Figura 4.2. Como podemos observar, não há indícios de afastamento da suposição de que os erros têm distribuição KwN. Fizemos o gráfico normal de probabilidade dos resíduos deviance modificado com envelope simulado para os demais modelos, mas não obtivemos um bom ajuste dos dados a esses modelos.

Na Figura 4.3, temos o gráfico de influência local considerando o esquema de perturbação ponderação de casos. Identificamos as observações \#26 e \#91 como possivelmente influentes. Com o intuito de analisar o impacto dessas observações nas estimativas dos parâmetros, realizamos uma análise confirmatória reajustando o modelo sem as observações consideradas influentes. Depois calculamos a mudança relativa em porcentagem das estimativas para os parâmetros do modelo.

As mudanças relativas para as observações \#26 e \#91, individualmente e conjuntamente, estão apresentadas na Tabela 4.3.

Apresentamos na Tabela 4.4 as estimativas dos parâmetros do modelo de regressão KwN sem essas observações. Notemos que a retirada das observações \#26 ou \#91 não causou mudanças inferenciais no modelo. Assim, sugerimos que essas observações não sejam eliminadas. 
Tabela 4.1: Estimativas de máxima verossimilhança e respectivos erros padrão dos parâmetros para os modelos de regressão $K w N$, LTII-N, Exp-N e normal ajustados aos dados de transplante de coração em Stanford

\begin{tabular}{c|ccccccc}
\hline \hline \multirow{2}{*}{ Distribuição } & \multicolumn{7}{c}{ Parâmetros } \\
\cline { 2 - 8 } & $\mathrm{a}$ & $\mathrm{b}$ & $\sigma$ & $\beta_{0}$ & $\beta_{1}$ & $\beta_{2}$ & $\beta_{3}$ \\
\hline \multirow{2}{*}{ KwN } & 0,0057 & 0,6564 & 0,2059 & 8,9446 & $-0,0708$ & 2,3957 & 2,1499 \\
& $(0,00085)$ & $(0,2269)$ & $(0,1130)$ & $(0,6190)$ & $(0,0158)$ & $(0,7923)$ & $(0,3398)$ \\
LTII-N & 1 & 0,0925 & 0,6171 & 5,5327 & $-0,0972$ & 1,2372 & 2,1327 \\
& - & $(0,0385)$ & $(0,0083)$ & $(0,0063)$ & $(0,0136)$ & $(0,5093)$ & $(0,0242)$ \\
Exp-N & 0,0053 & 1 & 0,2831 & 5,0512 & 0,0722 & 1,1427 & 1,3541 \\
& $(0,0006)$ & - & $(0,2152)$ & $(0,5112)$ & $(0,0173)$ & $(0,7946)$ & $(0,4989)$ \\
Normal & 1 & 1 & 1,1930 & 5,4570 & $-0,0577$ & 0,8706 & 2,6236 \\
& - & - & $(0,0717)$ & $(0,6105)$ & $(0,0132)$ & $(0,3562)$ & $(0,2644)$ \\
\hline \hline
\end{tabular}

Tabela 4.2: Critérios de informação

\begin{tabular}{c|ccc}
\hline \hline \multirow{2}{*}{ Distribuição } & \multicolumn{3}{|c}{ Critérios } \\
\cline { 2 - 4 } & AIC & CAIC & BIC \\
\hline KwN & 131,555 & 132,734 & 149,998 \\
LTII-N & 306,275 & 307,150 & 322,083 \\
Exp-N & 222,806 & 223,681 & 238,614 \\
Normal & 397,633 & 398,509 & 413,442 \\
\hline \hline
\end{tabular}

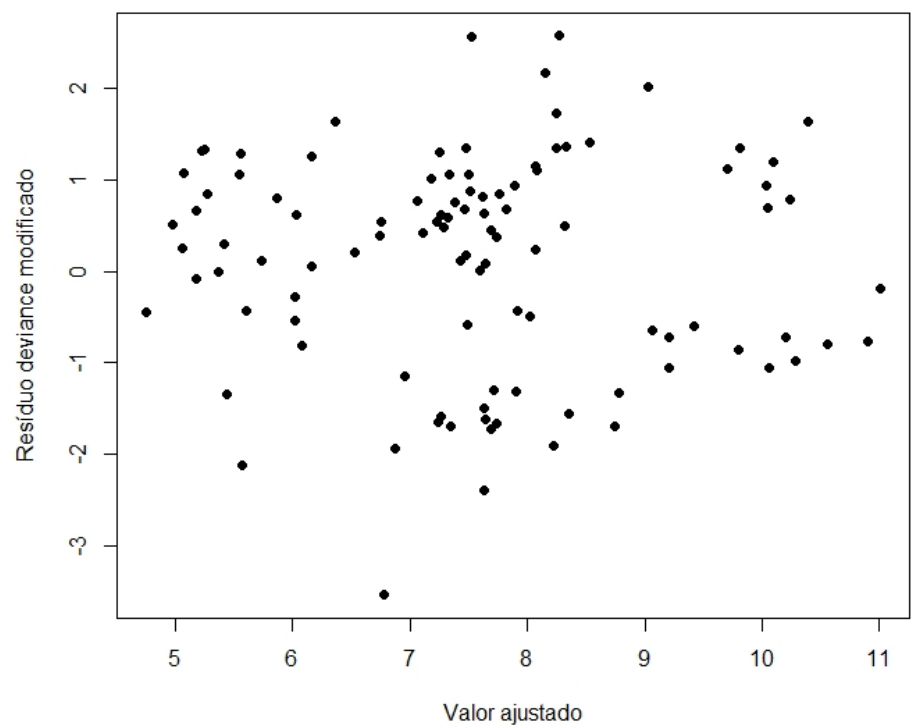

Figura 4.1: Gráficos dos resíduos deviance modificado versus valores ajustados para o modelo de regressão $K w N$. 


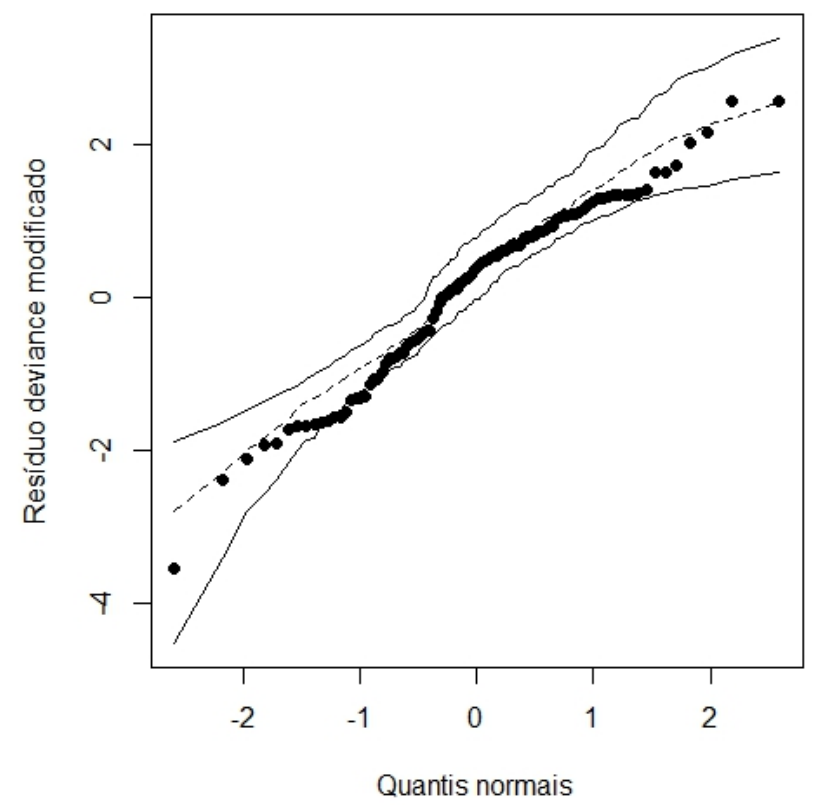

Figura 4.2: Gráfico de probabilidade normal com envelope simulado dos resíduos deviance modificado para o modelo de regressão $K w N$ com censura.

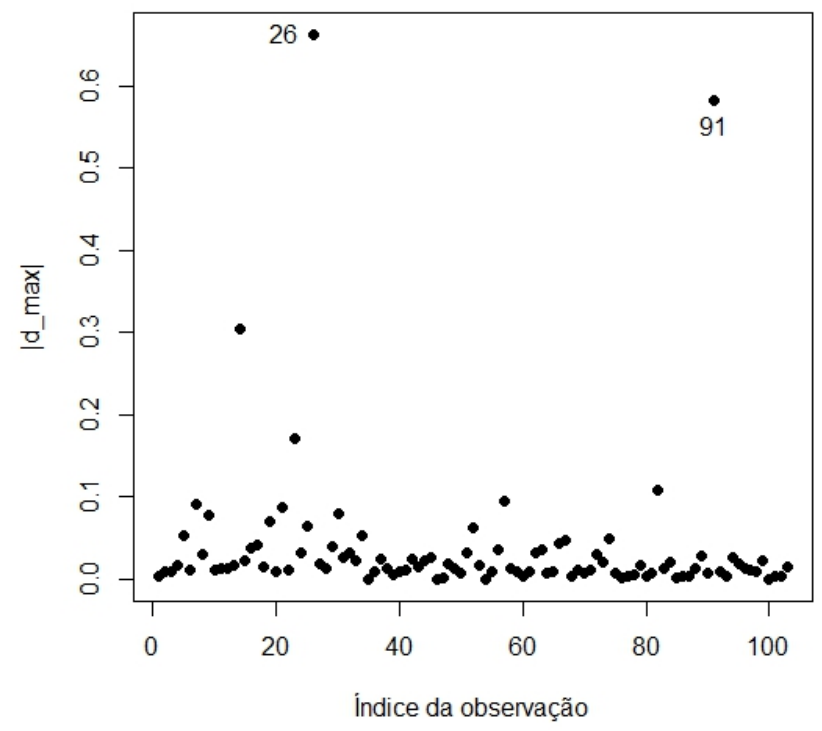

Figura 4.3: Gráfico de $\left|d_{\max }\right|$ contra índice da observação considerando o esquema de perturbação da ponderação de casos. 
Tabela 4.3: Mudanças relativas

\begin{tabular}{c|ccccccc}
\hline \hline \multirow{2}{*}{ Observações } & \multicolumn{7}{|c}{ Parâmetros } \\
\cline { 2 - 8 } & $a$ & $b$ & $\sigma$ & $\beta_{0}$ & $\beta_{1}$ & $\beta_{2}$ & $\beta_{3}$ \\
\hline$\# 26$ & $109,77 \%$ & $75,44 \%$ & $15,57 \%$ & $4,32 \%$ & $12,49 \%$ & $13,36 \%$ & $7,17 \%$ \\
$\# 91$ & $29,14 \%$ & $17,44 \%$ & $0,68 \%$ & $0,31 \%$ & $8,60 \%$ & $5,90 \%$ & $28,43 \%$ \\
$\# 26$ e \#91 & $70,82 \%$ & $16,38 \%$ & $8,01 \%$ & $3,85 \%$ & $48,22 \%$ & $13,10 \%$ & $46,31 \%$ \\
\hline \hline
\end{tabular}

Tabela 4.4: Estimativas de máxima verossimilhança e respectivos erros padrão dos parâmetros para os modelos de regressão KwN, ajustados aos dados de transplante de coração em Stanford sem as observações \#26 e \#91

\begin{tabular}{c|ccccccc}
\hline \hline \multirow{2}{*}{ Observações } & \multicolumn{7}{|c}{ Parâmetros } \\
\cline { 2 - 8 }$\#$ \#26 & $a$ & $b$ & $\sigma$ & $\beta_{0}$ & $\beta_{1}$ & $\beta_{2}$ & $\beta_{3}$ \\
\hline & 0,0119 & 1,1516 & 0,2380 & 8,5579 & $-0,0619$ & 2,7159 & 1,9958 \\
& $(0,00071)$ & $(0,2154)$ & $(0,1023)$ & $(0,6552)$ & $(0,0144)$ & $(0,5939)$ & $(0,2361)$ \\
\hline$\# 91$ & 0,0072 & 0,5419 & 0,2061 & 8,9720 & $-0,0768$ & 2,2544 & 2,7612 \\
& $(0,00081)$ & $(0,1333)$ & $(0,0209)$ & $(0,6014)$ & $(0,0153)$ & $(0,7871)$ & $(0,3060)$ \\
\hline$\# 26$ e \#91 & 0,0097 & 0,7639 & 0,1895 & 9,2893 & $-0,1049$ & 2,7093 & 3,1455 \\
& $(0,00073)$ & $(0,2213)$ & $(0,1114)$ & $(0,5988)$ & $(0,0109)$ & $(0,7712)$ & $(0,1679)$ \\
\hline \hline
\end{tabular}




\section{Capítulo 5}

\section{Considerações finais}

No desenvolvimento deste trabalho, estudamos a distribuição Kumaraswamy normal (KwN) gerada a partir da classe de distribuições Kumaraswamy generalizadas, proposta por Cordeiro e de Castro (2011). Apresentamos algumas propriedades desta distribuição, tais como, momentos ordinários, centrais, incompletos e ponderados por probabilidade, desvios médios, funções quantílica e geradora de momentos, estatísticas de ordem e dois tipos de entropias, Rényi e Shannon. Os parâmetros da distribuição são estimados por máxima verossimilhança. Por meio de estudos de simulação, constatou-se um desempenho satisfatório para a distribuição em questão. A utilidade da distribuição KwN é ilustrada por meio de uma aplicação de um conjunto de dados reais. Os critérios AIC, BIC e CAIC e o teste da razão de verossimilhanças, mostram que essa distribuição proporciona um ajuste mais adequado do que outras distribuições. Propomos dois modelos de regressão KwN, um com a presença de censura e outro com ausência e fornecemos algumas medidas diagnósticas para ambos os modelos. Duas aplicações dos novos modelos de regressão a dados reais comprovam empiricamente que eles podem ser usados de forma bastante eficaz para proporcionar ajustes melhores do que outros modelos bem conhecidos.

As principais contribuições científicas desta tese concentram-se nos Capítulos 2, 3 e 4 .

\subsection{Pesquisas futuras}

Dando continuidade a esta pesquisa, podemos futuramente

- Desenvolver um estudo sobre a assimetria da distribuição dos estimadores dos parâmetros $a$ e $b$ da distribuição discutida no Capitulo 2;

- Propor um modelo de regressão quantílica;

- Desenvolver e implementar computacionalmente outros métodos de estimação para os parâmetros do modelo de regressão KwN, por exemplo, o algoritmo de estimação EM. 


\section{Apêndice A}

\section{A.1 Elementos da matriz de informação observada $J(\theta)$}

Diferenciando (2.39) em relação aos elementos de $\boldsymbol{\theta}$, os elementos da matriz de informação observada $J(\theta)$ para os parâmetros $(a, b, \mu, \sigma)$ são:

$$
\begin{aligned}
& J_{a a}=-\frac{n}{a^{2}}-(b-1) \sum_{i=1}^{n} \frac{\left[\Phi\left(z_{i}\right)\right]^{a}\left\{\log \left[\Phi\left(z_{i}\right)\right]\right\}^{2}}{\left[1-\Phi\left(z_{i}\right)^{a}\right]}\left\{1-\frac{\left[\Phi\left(z_{i}\right)\right]^{a}}{\left[1-\Phi\left(z_{i}\right)^{a}\right]}\right\}, \\
& J_{a b}=-\sum_{i=1}^{n} \frac{\left[\Phi\left(z_{i}\right)\right]^{a}\left\{\log \left[\Phi\left(z_{i}\right)\right]\right\}}{\left[1-\Phi\left(z_{i}\right)^{a}\right]} \\
& J_{a \mu}=-\sum_{i=1}^{n} \sigma^{-1} \phi\left(z_{i}\right)\left\{\frac{1}{\Phi\left(z_{i}\right)}+\frac{(b-1)\left[\Phi\left(z_{i}\right)\right]^{a-1}}{1-\Phi\left(z_{i}\right)^{a}}\left\{1+a \log \left[\Phi\left(z_{i}\right)\right]-\frac{\left[\Phi\left(z_{i}\right)\right]^{a}}{1-\Phi\left(z_{i}\right)^{a}}\right.\right. \\
& \left.\left.\times \log \left[\Phi\left(z_{i}\right)\right]\right\}\right\} \\
& J_{a \sigma}=-\sum_{i=1}^{n}\left\{\frac{z_{i} \phi\left(z_{i}\right)}{\sigma\left[\Phi\left(z_{i}\right)\right]}-(b-1) \frac{\phi\left(z_{i}\right)\left[\Phi\left(z_{i}\right)\right]^{a-1}}{\sigma\left[1-\Phi\left(z_{i}\right)\right]^{a}}\left[z_{i}-a z_{i} \log \left[\Phi\left(z_{i}\right)\right]-a \Phi\left(z_{i}\right)^{a}\right.\right. \\
& \left.\left.\times \frac{\log \left[\Phi\left(z_{i}\right)\right]}{\sigma\left[1-\Phi\left(z_{i}\right)^{a}\right]}\right]\right\} \\
& J_{b b}=-\frac{n}{b^{2}} \\
& J_{b \mu}=-a \sum_{i=1}^{n} \frac{\left[\Phi\left(z_{i}\right)\right]^{a-1} \phi\left(\left(z_{i}\right)\right)}{\sigma\left[1-\Phi\left(z_{i}\right)^{a}\right]} \\
& J_{b \sigma}=a \sum_{i=1}^{n} \frac{z_{i}\left[\Phi\left(z_{i}\right)\right]^{a-1} \phi\left(z_{i}\right)}{\sigma\left[1-\Phi\left(z_{i}\right)^{a}\right]}, \\
& J_{\mu \mu}=-\sum_{i=1}^{n}\left\{\frac{1}{\sigma^{2}}+\frac{(a-1) \phi\left(z_{i}\right)}{\sigma^{2} \Phi\left(z_{i}\right)}\left(z_{i}-\frac{\phi\left(z_{i}\right)}{\Phi\left(z_{i}\right)}\right)-\frac{a(b-1) \phi\left(z_{i}\right) \Phi\left(z_{i}\right)^{a-1}}{\sigma^{2}\left[1-\Phi\left(z_{i}\right)^{a}\right]}[(a-1)\right. \\
& \left.\left.\times\left[\Phi\left(z_{i}\right)\right]^{-1}+z_{i}+\frac{a \Phi\left(z_{i}\right)^{a-1} \phi\left(z_{i}\right)}{\sigma^{2}\left[1-\Phi\left(z_{i}\right)^{a}\right]}\right]\right\},
\end{aligned}
$$




$$
\begin{aligned}
J_{\mu \sigma}= & -\sum_{i=1}^{n}\left\{\frac{2 z_{i}}{\sigma}+\frac{(a-1) \phi\left(z_{i}\right) z_{i}}{\sigma^{2} \Phi\left(z_{i}\right)}\left[z_{i}+\phi\left(z_{i}\right)-\frac{\Phi\left(z_{i}\right)}{\left[\Phi\left(z_{i}\right)\right]}\right]+\frac{a(b-1) \Phi\left(z_{i}\right)^{a-1} \phi\left(z_{i}\right)}{\sigma^{2}\left[1-\Phi\left(z_{i}\right)^{a}\right]} \times\right. \\
& \left.\left\{z_{i}+\frac{(a-1)\left[\Phi\left(z_{i}\right)\right]^{-1}}{\sigma}-\frac{z_{i} \phi\left(z_{i}\right)\left[\Phi\left(z_{i}\right)\right]^{a-1}}{\sigma^{2}\left[1-\Phi\left(z_{i}\right)^{a}\right]}-\frac{\phi\left(z_{i}\right)\left[1-\Phi\left(z_{i}\right)^{a}\right]}{\sigma^{2}\left[1-\Phi\left(z_{i}\right)^{a}\right]}\right\}\right\}
\end{aligned}
$$

e

$$
\begin{aligned}
J_{\sigma \sigma}= & \frac{n}{\sigma^{2}}+\sum_{i=1}^{n}\left\{\frac{(a-1) z_{i} \phi\left(z_{i}\right)}{\sigma^{2} \Phi\left(z_{i}\right)}\left\{1-z_{i}^{2}-\frac{z_{i} \phi\left(z_{i}\right)}{\Phi\left(z_{i}\right)}-\frac{\phi\left(z_{i}\right)}{\Phi\left(z_{i}\right)}\right\}-\frac{\phi\left(z_{i}\right) \Phi\left(z_{i}\right)^{a-1}}{\sigma\left[1-\Phi\left(z_{i}\right)^{a}\right]}\right. \\
& a(b-1) z_{i}\left\{\sigma^{-2}-z_{i}+\sigma^{-1}(a-1) z_{i} \phi\left(z_{i}\right) \Phi\left(z_{i}\right)^{-1}+\frac{a\left[1-\Phi\left(z_{i}\right)^{a}\right]}{\sigma\left[1-\Phi\left(z_{i}\right)^{a}\right]}-z_{1} \phi\left(z_{i}\right) \times\right. \\
& \left.\left.\frac{\Phi\left(z_{i}\right)^{a-1}}{\sigma\left[1-\Phi\left(z_{i}\right)^{a}\right]}\right\}\right\} .
\end{aligned}
$$




\section{Apêndice B}

\section{A.2 Elementos da matriz de informação observada $J(\eta)$ - Modelo de regressão $\mathrm{KwN}$}

Diferenciando (3.2) em relação aos elementos de $\boldsymbol{\eta}$, os elementos da matriz de informação observada $J(\eta)$ para os parâmetros $\left(a, b, \sigma, \boldsymbol{\beta}^{\top}\right)$ são:

$$
\begin{aligned}
& J_{a a}=-\frac{n}{a^{2}}-(b-1) \sum_{i=1}^{n} \frac{\left[\Phi\left(z_{i}\right)\right]^{a}\left\{\log \left[\Phi\left(z_{i}\right)\right]\right\}^{2}}{\left[1-\Phi\left(z_{i}\right)^{a}\right]}\left\{1-\frac{\left[\Phi\left(z_{i}\right)\right]^{a}}{1-\Phi\left(z_{i}\right)^{a}}\right\} \\
& J_{a b}=-\sum_{i=1}^{n} \frac{\left[\Phi\left(z_{i}\right)\right]^{a}\left\{\log \left[\Phi\left(z_{i}\right)\right]\right\}}{\left[1-\Phi\left(z_{i}\right)^{a}\right]} \\
& J_{a \beta_{j}}=-\sum_{i=1}^{n} \sigma^{-1} \phi\left(z_{i}\right)\left\{\frac{1}{\Phi\left(z_{i}\right)}+\frac{(b-1)\left[\Phi\left(z_{i}\right)\right]^{a-1}}{1-\Phi\left(z_{i}\right)^{a}}\left\{1+a \log \left[\Phi\left(z_{i}\right)\right]-\frac{\left[\Phi\left(z_{i}\right)\right]^{a} \log \left[\Phi\left(z_{i}\right)\right]}{1-\Phi\left(z_{i}\right)^{a}}\right\}\right\}, \\
& J_{a \sigma}=-\sum_{i=1}^{n}\left\{\frac{z_{i} \phi\left(z_{i}\right)}{\sigma\left[\Phi\left(z_{i}\right)\right]}-(b-1) \frac{\phi\left(z_{i}\right)\left[\Phi\left(z_{i}\right)\right]^{a-1}}{\sigma\left[1-\Phi\left(z_{i}\right)\right]^{a}}\left[z_{i}-a z_{i} \log \left[\Phi\left(z_{i}\right)\right]-\frac{a \Phi\left(z_{i}\right)^{a} \log \left[\Phi\left(z_{i}\right)\right]}{\sigma\left[1-\Phi\left(z_{i}\right)^{a}\right]}\right]\right\} \\
& J_{b b}=-\frac{n}{b^{2}}, \\
& J_{b \beta_{j}}=-a \sum_{i=1}^{n} \frac{\left[\Phi\left(z_{i}\right)\right]^{a-1} \phi\left(\left(z_{i}\right)\right)}{\sigma\left[1-\Phi\left(z_{i}\right)^{a}\right]} \\
& J_{b \sigma}=a \sum_{i=1}^{n} \frac{z_{i}\left[\Phi\left(z_{i}\right)\right]^{a-1} \phi\left(z_{i}\right)}{\sigma\left[1-\Phi\left(z_{i}\right)^{a}\right]} \\
& J_{\beta_{j} \beta_{k}}=-\sum_{i=1}^{n}\left\{\frac{1}{\sigma^{2}}+\frac{(a-1) \phi\left(z_{i}\right)}{\sigma^{2} \Phi\left(z_{i}\right)}\left(z_{i}-\frac{\phi\left(z_{i}\right)}{\Phi\left(z_{i}\right)}\right)-\frac{a(b-1) \phi\left(z_{i}\right) \Phi\left(z_{i}\right)^{a-1}}{\sigma^{2}\left[1-\Phi\left(z_{i}\right)^{a}\right]}\left[(a-1)\left[\Phi\left(z_{i}\right)\right]^{-1}\right.\right. \\
& \left.\left.+z_{i}+\frac{a \Phi\left(z_{i}\right)^{a-1} \phi\left(z_{i}\right)}{\sigma^{2}\left[1-\Phi\left(z_{i}\right)^{a}\right]}\right]\right\}
\end{aligned}
$$




$$
\begin{aligned}
J_{\beta_{j} \sigma}= & -\sum_{i=1}^{n}\left\{\frac{2 z_{i}}{\sigma}+\frac{(a-1) \phi\left(z_{i}\right) z_{i}}{\sigma^{2} \Phi\left(z_{i}\right)}\left[z_{i}+\phi\left(z_{i}\right)-\frac{\Phi\left(z_{i}\right)}{\left[\Phi\left(z_{i}\right)\right]}\right]+\frac{a(b-1) \Phi\left(z_{i}\right)^{a-1} \phi\left(z_{i}\right)}{\sigma^{2}\left[1-\Phi\left(z_{i}\right)^{a}\right]}\right. \\
& \left.\times\left\{z_{i}+\frac{(a-1)\left[\Phi\left(z_{i}\right)\right]^{-1}}{\sigma}-\frac{z_{i} \phi\left(z_{i}\right)\left[\Phi\left(z_{i}\right)\right]^{a-1}}{\sigma^{2}\left[1-\Phi\left(z_{i}\right)^{a}\right]}-\frac{\phi\left(z_{i}\right)\left[1-\Phi\left(z_{i}\right)^{a}\right]}{\sigma^{2}\left[1-\Phi\left(z_{i}\right)^{a}\right]}\right\}\right\},
\end{aligned}
$$

e

$$
\begin{aligned}
J_{\sigma \sigma}= & \frac{n}{\sigma^{2}}+\sum_{i=1}^{n}\left(\frac{(a-1) z_{i} \phi\left(z_{i}\right)}{\sigma^{2} \Phi\left(z_{i}\right)}\left\{1-z_{i}^{2}-\frac{z_{i} \phi\left(z_{i}\right)}{\Phi\left(z_{i}\right)}-\frac{\phi\left(z_{i}\right)}{\Phi\left(z_{i}\right)}\right\}-\frac{a(b-1) z_{i} \phi\left(z_{i}\right) \Phi\left(z_{i}\right)^{a-1}}{\sigma\left[1-\Phi\left(z_{i}\right)^{a}\right]}\left\{\sigma^{-2}\right.\right. \\
& \left.\left.-z_{i}+\sigma^{-1}(a-1) z_{i} \phi\left(z_{i}\right) \Phi\left(z_{i}\right)^{-1}+\frac{a\left[1-\Phi\left(z_{i}\right)^{a}\right]}{\sigma\left[1-\Phi\left(z_{i}\right)^{a}\right]}-\frac{z_{1} \phi\left(z_{i}\right) \Phi\left(z_{i}\right)^{a-1}}{\sigma\left[1-\Phi\left(z_{i}\right)^{a}\right]}\right\}\right) .
\end{aligned}
$$




\section{Apêndice C}

\section{A.3 Elementos da matriz de informação observada $J(\eta)$-Modelo de regressão $\mathrm{KwN}$ com censura}

Diferenciando (4.3) em relação aos elementos de $\boldsymbol{\eta}$, os elementos da matriz de informação observada $J(\eta)$ para os parâmetros $\left(a, b, \sigma, \boldsymbol{\beta}^{\top}\right)$ são:

$$
\begin{aligned}
& J_{a a}=-\frac{n}{a^{2}}-(b-1) \sum_{i \in F} \frac{\left[\Phi\left(z_{i}\right)\right]^{a}\left\{\log \left[\Phi\left(z_{i}\right)\right]\right\}^{2}}{\left[1-\Phi\left(z_{i}\right)^{a}\right]}\left\{1-\frac{\left[\Phi\left(z_{i}\right)\right]^{a}}{1-\Phi\left(z_{i}\right)^{a}}\right\}-b \sum_{i \in C} \frac{\left[\Phi\left(z_{i}\right)\right]^{a}\left[\log \Phi\left(z_{i}\right)\right]^{2}}{\left[1-\Phi\left(z_{i}\right)^{a}\right]} \\
& \times\left\{1+\frac{\left[\Phi\left(z_{i}\right)\right]^{a}}{\left[1-\Phi\left(z_{i}\right)^{a}\right]}\right\}, \\
& J_{a b}=-\sum_{i \in F} \frac{\left[\Phi\left(z_{i}\right)\right]^{a}\left\{\log \left[\Phi\left(z_{i}\right)\right]\right\}}{\left[1-\Phi\left(z_{i}\right)^{a}\right]}-\sum_{i \in C} \frac{\left[\Phi\left(z_{i}\right)\right]^{a}\left[\log \Phi\left(z_{i}\right)\right]}{1-\Phi\left(z_{i}\right)^{a}}, \\
& J_{a \beta_{j}=}=-\sum_{i \in F} \sigma^{-1} \phi\left(z_{i}\right)\left\{\frac{1}{\Phi\left(z_{i}\right)}+\frac{(b-1)\left[\Phi\left(z_{i}\right)\right]^{a-1}}{1-\Phi\left(z_{i}\right)^{a}}\left\{1+a \log \left[\Phi\left(z_{i}\right)\right]-\frac{\left[\Phi\left(z_{i}\right)\right]^{a} \log \left[\Phi\left(z_{i}\right)\right]}{\left.\left.1-\Phi\left(z_{i}\right)^{a}\right\}\right\}}\right.\right. \\
&+b \sum_{i \in C} \frac{x_{i j}\left[\Phi\left(z_{i}\right)\right]^{a-1} \phi\left(z_{i}\right)}{\sigma\left[1-\Phi\left(z_{i}\right)^{a}\right]}\left\{1+a \log \left[\Phi\left(z_{i}\right)\right]+\frac{a\left[\Phi\left(z_{i}\right)\right]^{a-1}\left\{\log \left[\Phi\left(z_{i}\right)\right]\right\}}{\left[1-\Phi\left(z_{i}\right)^{a}\right]}\right\} \\
& J_{a \sigma}=-\sum_{i=1}^{n}\left\{\frac{z_{i} \phi\left(z_{i}\right)}{\sigma\left[\Phi\left(z_{i}\right)\right]}-(b-1) \frac{\phi\left(z_{i}\right)\left[\Phi\left(z_{i}\right)\right]^{a-1}}{\sigma\left[1-\Phi\left(z_{i}\right)\right]^{a}}\left[z_{i}-a z_{i} \log \left[\Phi\left(z_{i}\right)\right]-\frac{a \Phi\left(z_{i}\right)^{a} \log \left[\Phi\left(z_{i}\right)\right]}{\sigma\left[1-\Phi\left(z_{i}\right)^{a}\right]}\right]\right\} \\
&+b \sum_{i \in C} \frac{z_{i}\left[\Phi\left(z_{i}\right)\right]^{a-1} \phi\left(z_{i}\right)}{\sigma\left[1-\Phi\left(z_{i}\right)^{a}\right]}\left\{1+a \log \left[\Phi\left(z_{i}\right)\right]+\frac{a\left[\Phi\left(z_{i}\right)\right]^{a-1}\left\{\log \left[\Phi\left(z_{i}\right)\right]\right\}}{\left[1-\Phi\left(z_{i}\right)^{a}\right]}\right\}, \\
& L_{b \sigma}=a \sum_{i=1}^{n} \frac{z_{i}\left[\Phi\left(z_{i}\right)\right]^{a-1} \phi\left(z_{i}\right)}{\sigma\left[1-\Phi\left(z_{i}\right)^{a}\right]}+a \sum_{i \in C} \frac{z_{i}\left[\Phi\left(z_{i}\right)\right]^{a-1} \phi\left(z_{i}\right)}{\sigma\left[1-\Phi\left(z_{i}\right)^{a}\right]}, \\
& J_{b \beta_{j}}=-a \sum_{b b}^{n} \frac{\left[\Phi\left(z_{i}\right)\right]^{a-1} \phi\left(\left(z_{i}\right)\right)}{\sigma\left[1-\Phi\left(z_{i}\right)^{a}\right]}+a \sum_{i \in C} \frac{x_{i j}\left[\Phi\left(z_{i}\right)\right]^{a-1} \phi\left(z_{i}\right)}{\sigma\left[1-\Phi\left(z_{i}\right)^{a}\right]}, \\
& b^{2},
\end{aligned}
$$




$$
\begin{aligned}
J_{\beta_{j} \beta_{k}}= & -\sum_{i=1}^{n}\left\{\frac{1}{\sigma^{2}}+\frac{(a-1) \phi\left(z_{i}\right)}{\sigma^{2} \Phi\left(z_{i}\right)}\left(z_{i}-\frac{\phi\left(z_{i}\right)}{\Phi\left(z_{i}\right)}\right)-\frac{a(b-1) \phi\left(z_{i}\right) \Phi\left(z_{i}\right)^{a-1}}{\sigma^{2}\left[1-\Phi\left(z_{i}\right)^{a}\right]}\left[(a-1)\left[\Phi\left(z_{i}\right)\right]^{-1}\right.\right. \\
& \left.\left.+z_{i}+\frac{a \Phi\left(z_{i}\right)^{a-1} \phi\left(z_{i}\right)}{\sigma^{2}\left[1-\Phi\left(z_{i}\right)^{a}\right]}\right]\right\}+a b \sum_{i \in C} \frac{\Phi\left(z_{i}\right)^{a-1} \phi\left(z_{i}\right) x_{i j}}{\sigma\left[1-\Phi\left(z_{i}\right)^{a}\right]}\left\{\frac{(a-1) \Phi\left(z_{i}\right)^{-1} \phi\left(z_{i}\right) x_{i j}}{\sigma}-x_{i j}\right. \\
& \left.\times \frac{x_{i k} z_{i}}{\sigma}-\frac{a \Phi\left(z_{i}\right)^{a-1} \phi\left(z_{i}\right) x_{i j} x_{i k}}{\sigma\left[1-\Phi\left(z_{i}\right)^{a}\right]}\right\}, \\
J_{\beta_{j} \sigma}= & -\sum_{i=1}^{n}\left(\frac{2 z_{i}}{\sigma}+\frac{(a-1) \phi\left(z_{i}\right) z_{i}}{\sigma^{2} \Phi\left(z_{i}\right)}\left[z_{i}+\phi\left(z_{i}\right)-\frac{\Phi\left(z_{i}\right)}{\left[\Phi\left(z_{i}\right)\right]}\right]+\frac{a(b-1) \Phi\left(z_{i}\right)^{a-1} \phi\left(z_{i}\right)}{\sigma^{2}\left[1-\Phi\left(z_{i}\right)^{a}\right]}\right. \\
& \left.\times\left\{z_{i}+\frac{(a-1)\left[\Phi\left(z_{i}\right)\right]^{-1}}{\sigma}-\frac{z_{i} \phi\left(z_{i}\right)\left[\Phi\left(z_{i}\right)\right]^{a-1}}{\sigma^{2}\left[1-\Phi\left(z_{i}\right)^{a}\right]}-\frac{\phi\left(z_{i}\right)\left[1-\Phi\left(z_{i}\right)^{a}\right]}{\sigma^{2}\left[1-\Phi\left(z_{i}\right)^{a}\right]}\right\}\right)-a b \sum_{i \in C} \frac{\phi\left(z_{i}\right)}{\sigma^{2}} \\
& \times \frac{\Phi\left(z_{i}\right)^{a-1} x_{i j}}{\left[1-\Phi\left(z_{i}\right)^{a}\right]}\left\{(a-1) \Phi\left(z_{i}\right)^{-1} x_{i j} z_{i}-x_{i j} z_{i}^{2}-x_{i j}+\frac{a \Phi\left(z_{i}\right)^{a-1} \phi\left(z_{i}\right) x_{i j} z_{i}}{\left[1-\Phi\left(z_{i}\right)^{a}\right]}\right\},
\end{aligned}
$$

e

$$
\begin{aligned}
J_{\sigma \sigma}= & \frac{n}{\sigma^{2}}+\sum_{i=1}^{n}\left(\frac{(a-1) z_{i} \phi\left(z_{i}\right)}{\sigma^{2} \Phi\left(z_{i}\right)}\left\{1-z_{i}^{2}-\frac{z_{i} \phi\left(z_{i}\right)}{\Phi\left(z_{i}\right)}-\frac{\phi\left(z_{i}\right)}{\Phi\left(z_{i}\right)}\right\}-\frac{a(b-1) z_{i} \phi\left(z_{i}\right) \Phi\left(z_{i}\right)^{a-1}}{\sigma\left[1-\Phi\left(z_{i}\right)^{a}\right]}\left\{\sigma^{-2}\right.\right. \\
& \left.\left.-z_{i}+\sigma^{-1}(a-1) z_{i} \phi\left(z_{i}\right) \Phi\left(z_{i}\right)^{-1}+\frac{a\left[1-\Phi\left(z_{i}\right)^{a}\right]}{\sigma\left[1-\Phi\left(z_{i}\right)^{a}\right]}-\frac{z_{1} \phi\left(z_{i}\right) \Phi\left(z_{i}\right)^{a-1}}{\sigma\left[1-\Phi\left(z_{i}\right)^{a}\right]}\right\}\right)+b a \sum_{i \in C} \phi\left(z_{i}\right) \\
& \times(a-1) \frac{\Phi\left(z_{i}\right)^{a-1} z_{i}^{2}}{\sigma^{2}\left[1-\Phi\left(z_{i}\right)^{a}\right]} .
\end{aligned}
$$




\section{Apêndice D}

\section{A.4 Dados de transplante de coração em Stanford}

Tabela A.1: Dados de transplante de coração em Stanford

\begin{tabular}{|c|c|c|c|c|}
\hline Idade de aceitação & Cirurgia prévia & Transplante & Censura & tempo de sobrevida (dias) \\
\hline 30,84 & 0 & 0 & 1 & 50 \\
\hline 51,84 & 0 & 0 & 1 & 6 \\
\hline 54,30 & 0 & 1 & 1 & 16 \\
\hline 40,26 & 0 & 1 & 1 & 39 \\
\hline 20,79 & 0 & 0 & 1 & 18 \\
\hline 54,60 & 0 & 0 & 1 & 3 \\
\hline 50,87 & 0 & 1 & 1 & 675 \\
\hline 45,35 & 0 & 0 & 1 & 40 \\
\hline 47,16 & 0 & 0 & 1 & 85 \\
\hline 42,50 & 0 & 1 & 1 & 58 \\
\hline 47,98 & 0 & 1 & 1 & 153 \\
\hline 53,19 & 0 & 0 & 1 & 8 \\
\hline 54,57 & 0 & 1 & 1 & 81 \\
\hline 54,01 & 0 & 1 & 1 & 1387 \\
\hline 53,82 & 1 & 0 & 1 & 1 \\
\hline 49,45 & 0 & 1 & 1 & 308 \\
\hline 20,33 & 0 & 0 & 1 & 36 \\
\hline 56,85 & 0 & 1 & 1 & 43 \\
\hline 59,12 & 0 & 0 & 1 & 37 \\
\hline 55,28 & 0 & 1 & 1 & 28 \\
\hline 43,34 & 0 & 1 & 1 & 1032 \\
\hline 42,78 & 0 & 1 & 1 & 51 \\
\hline 58,36 & 0 & 1 & 1 & 733 \\
\hline 51,80 & 0 & 1 & 1 & 219 \\
\hline 33,22 & 0 & 1 & 0 & 1800 \\
\hline 30,54 & 0 & 0 & 0 & 1401 \\
\hline 8,79 & 0 & 0 & 1 & 263 \\
\hline 54,02 & 0 & 1 & 1 & 72 \\
\hline 50,43 & 0 & 0 & 1 & 35 \\
\hline 44,91 & 0 & 1 & 1 & 852 \\
\hline 54,89 & 0 & 0 & 1 & 16 \\
\hline 64,41 & 0 & 1 & 1 & 77 \\
\hline
\end{tabular}


Tabela A.2: Continuação da Tabela anterior

\begin{tabular}{|c|c|c|c|c|}
\hline Idade de aceitação & Cirurgia prévia & Transplante & Censura & tempo de sobrevida (dias) \\
\hline 48,90 & 0 & 1 & 0 & 1587 \\
\hline 40,55 & 0 & 1 & 0 & 1572 \\
\hline 43,47 & 0 & 0 & 1 & 12 \\
\hline 48,93 & 0 & 1 & 1 & 100 \\
\hline 61,50 & 0 & 1 & 1 & 66 \\
\hline 41,47 & 0 & 1 & 1 & 5 \\
\hline 50,52 & 0 & 1 & 1 & 53 \\
\hline 48,48 & 1 & 1 & 0 & 1408 \\
\hline 45,30 & 1 & 1 & 0 & 1322 \\
\hline 36,44 & 0 & 0 & 1 & 3 \\
\hline 43,39 & 1 & 0 & 1 & 2 \\
\hline 42,58 & 1 & 0 & 1 & 40 \\
\hline 36,18 & 0 & 1 & 1 & 45 \\
\hline 48,61 & 1 & 1 & 1 & 996 \\
\hline 47,10 & 0 & 1 & 1 & 72 \\
\hline 56,04 & 0 & 0 & 1 & 9 \\
\hline 36,65 & 1 & 1 & 0 & 1142 \\
\hline 45,89 & 1 & 1 & 1 & 980 \\
\hline 48,73 & 0 & 1 & 1 & 285 \\
\hline 41,25 & 0 & 0 & 1 & 102 \\
\hline 47,34 & 0 & 1 & 1 & 188 \\
\hline 47,79 & 0 & 0 & 1 & 3 \\
\hline 52,45 & 0 & 1 & 1 & 61 \\
\hline 38,74 & 0 & 1 & 0 & 942 \\
\hline 41,26 & 0 & 0 & 1 & 149 \\
\hline 48,02 & 1 & 1 & 1 & 343 \\
\hline 41,38 & 1 & 1 & 0 & 916 \\
\hline 49,05 & 0 & 1 & 1 & 68 \\
\hline 52,56 & 0 & 0 & 1 & 2 \\
\hline 39,35 & 0 & 0 & 1 & 69 \\
\hline 32,66 & 0 & 1 & 0 & 842 \\
\hline 48,82 & 1 & 1 & 1 & 584 \\
\hline 51,29 & 0 & 1 & 1 & 78 \\
\hline 53,21 & 0 & 0 & 1 & 32 \\
\hline 19,55 & 0 & 1 & 1 & 285 \\
\hline 45,24 & 0 & 1 & 1 & 68 \\
\hline 47,99 & 0 & 1 & 0 & 670 \\
\hline 53,00 & 0 & 1 & 1 & 30 \\
\hline 47,41 & 0 & 1 & 0 & 620 \\
\hline 26,73 & 0 & 1 & 0 & 596 \\
\hline 56,33 & 0 & 1 & 1 & 90 \\
\hline 29,17 & 0 & 1 & 1 & 17 \\
\hline 52,18 & 0 & 0 & 1 & 2 \\
\hline 52,08 & 1 & 1 & 0 & 545 \\
\hline 41,11 & 0 & 0 & 1 & 21 \\
\hline 48,70 & 0 & 1 & 0 & 515 \\
\hline 53,78 & 0 & 1 & 1 & 96 \\
\hline 46,44 & 1 & 1 & 0 & 482 \\
\hline
\end{tabular}


Tabela A.3: Continuação da Tabela anterior

\begin{tabular}{c|c|c|c|c}
\hline Idade de aceitação & Cirurgia prévia & Transplante & Censura & tempo de sobrevida (dias) \\
\hline 52,89 & 0 & 1 & 0 & 445 \\
29,20 & 0 & 0 & 0 & 428 \\
53,31 & 0 & 1 & 1 & 80 \\
42,72 & 0 & 1 & 1 & 334 \\
47,98 & 0 & 0 & 1 & 5 \\
48,92 & 0 & 1 & 0 & 397 \\
46,25 & 0 & 1 & 1 & 110 \\
54,36 & 0 & 1 & 0 & 370 \\
51,05 & 0 & 1 & 1 & 207 \\
52,03 & 1 & 1 & 1 & 186 \\
47,59 & 0 & 0 & 1 & 340 \\
44,98 & 0 & 1 & 0 & 340 \\
47,75 & 0 & 1 & 0 & 265 \\
43,84 & 1 & 1 & 1 & 165 \\
40,28 & 0 & 1 & 1 & 16 \\
26,65 & 0 & 1 & 0 & 180 \\
23,62 & 0 & 1 & 0 & 131 \\
28,63 & 0 & 1 & 0 & 109 \\
49,83 & 0 & 0 & 1 & 21 \\
35,06 & 1 & 1 & 0 & 39 \\
49,52 & 0 & 0 & 0 & 31 \\
40,39 & 0 & 0 & 0 & 11 \\
39,32 & 0 & 0 & 1 & 6 \\
\hline
\end{tabular}




\title{
Referências Bibliográficas
}

\author{
Abramowitz e Stegun (1965) Milton Abramowitz e Irene A Stegun. Handbook of mathematical \\ functions: with formulas, graphs, and mathematical tables, volume 55. Courier Corporation. Citado \\ na pág. 13
}

Andrade et al. (2015) Thiago Andrade, Heloisa Rodrigues, Marcelo Bourguignon e Gauss Cordeiro. The exponentiated generalized gumbel distribution. Revista Colombiana de Estadistica, 38(1):123-143. Citado na pág. 2

Barreto-Souza et al. (2010) Wagner Barreto-Souza, Alessandro HS Santos e Gauss M Cordeiro. The beta generalized exponential distribution. Journal of Statistical Computation and Simulation, 80(2):159-172. Citado na pág. 1

Barros (2008) Angela Alves de Araújo Barros. Distribuições Exponencializada e Estendidas: Uma abordagem Clássica e Bayesiana. Tese de Doutorado, Universidade Federal Rural de Pernambuco. Citado na pág. 1

Carrasco et al. (2008) Jalmar MF Carrasco, Edwin MM Ortega e Gilberto A Paula. Log-modified weibull regression models with censored data: Sensitivity and residual analysis. Computational Statistics \& Data Analysis, 52(8):4021-4039. Citado na pág. 39

Castellares et al. (2013) F Castellares, LC Montenegro e GM Cordeiro. The beta log-normal distribution. Journal of Statistical Computation and Simulation, 83(2):203-228. Citado na pág. 23

Comtet () Louis Comtet. Advanced combinatorics. 1974. Reidel, Dordrecht. Citado na pág. 16

Cook (1977) R Dennis Cook. Detection of influential observation in linear regression. Technometrics, 19(1):15-18. Citado na pág. 31

Cook (1986) R Dennis Cook. Assessment of local influence. Journal of the Royal Statistical Society. Series B (Methodological), páginas 133-169. Citado na pág. 31

Cordeiro e de Castro (2011) Gauss M Cordeiro e Mario de Castro. A new family of generalized distributions. Journal of statistical computation and simulation, 81(7):883-898. Citado na pág. 1, 2, 5,19

Cordeiro (1999) Gauss Moutinho Cordeiro. Introdução à teoria Assintótica. IMPA. Citado na pág. 17

Cruz et al. (2016) José Nilton da Cruz, Edwin MM Ortega e Gauss M Cordeiro. The log-odd log-logistic weibull regression model: modelling, estimation, influence diagnostics and residual analysis. Journal of Statistical Computation and Simulation, 86(8):1516-1538. Citado na pág. 2

de Pascoa et al. (2011) Marcelino AR de Pascoa, Edwin MM Ortega e Gauss M Cordeiro. The kumaraswamy generalized gamma distribution with application in survival analysis. Statistical methodology, 8(5):411-433. Citado na pág. 2 
De Santana et al. (2012) Tiago Viana Flor De Santana, Edwin MM Ortega, Gauss M Cordeiro e Giovana O Silva. The kumaraswamy-log-logistic distribution. Journal of Statistical Theory and Applications, 11(3):265-291. Citado na pág. 2

Dunn e Smyth (1996) Peter K Dunn e Gordon K Smyth. Randomized quantile residuals. Journal of Computational and Graphical Statistics, 5(3):236-244. Citado na pág. 33

Eugene et al. (2002) Nicholas Eugene, Carl Lee e Felix Famoye. Beta-normal distribution and its applications. Communications in Statistics-Theory and methods, 31(4):497-512. Citado na pág. 1

Fleming e Harrington (2011) Thomas R Fleming e David P Harrington. Counting processes and survival analysis, volume 169. John Wiley \& Sons. Citado na pág. 43

Gradshteyn e Ryzhik (2008) IS Gradshteyn e IM Ryzhik. Table of integrals, series, and products. 2000. There is no corresponding record for this reference. Citado na pág. 11

Gupta et al. (1998) Ramesh C Gupta, Pushpa L Gupta e Rameshwar D Gupta. Modeling failure time data by lehman alternatives. Communications in Statistics-Theory and methods, 27(4): 887-904. Citado na pág. 1

Gupta e Kundu (1999) Rameshwar D Gupta e Debasis Kundu. Theory \& methods: Generalized exponential distributions. Australian \& New Zealand Journal of Statistics, 41(2):173-188. Citado na pág. 1

Gupta e Kundu (2001) Rameshwar D Gupta e Debasis Kundu. Generalized exponential distribution: different method of estimations. Journal of Statistical Computation and Simulation, 69 (4):315-337. Citado na pág. 2

Hashimoto et al. (2010) Elizabeth M Hashimoto, Edwin MM Ortega, Vicente G Cancho e Gauss M Cordeiro. The log-exponentiated weibull regression model for interval-censored data. Computational Statistics \&3 Data Analysis, 54(4):1017-1035. Citado na pág. 2

Kalbfleisch e Prentice (1980) JD Kalbfleisch e Ross L Prentice. statistical analysis of failure time data. Citado na pág. 43

Kenney e Keeping (1962) JF Kenney e ES Keeping. Moving averages. Mathematics of Statistics, 3:221-223. Citado na pág. 13

Lehmann (1953) Erich L Lehmann. The power of rank tests. The Annals of Mathematical Statistics, páginas 23-43. Citado na pág. 5

Leiva et al. (2007) Víctor Leiva, Michelli Barros, Gilberto A Paula e Manuel Galea. Influence diagnostics in log-birnbaum-saunders regression models with censored data. Computational Statistics ES Data Analysis, 51(12):5694-5707. Citado na pág. 39

Marshall e Olkin (1997) Albert W Marshall e Ingram Olkin. A new method for adding a parameter to a family of distributions with application to the exponential and weibull families. Biometrika, 84(3):641-652. Citado na pág. 1

Moors (1988) JJA Moors. A quantile alternative for kurtosis. The statistician, páginas 25-32. Citado na pág. 13

Mudholkar e Srivastava (1993) Govind S Mudholkar e Deo Kumar Srivastava. Exponentiated weibull family for analyzing bathtub failure-rate data. IEEE transactions on reliability, 42(2): 299-302. Citado na pág. 1

Mudholkar et al. (1995) Govind S Mudholkar, Deo Kumar Srivastava e Marshall Freimer. The exponentiated weibull family: a reanalysis of the bus-motor-failure data. Technometrics, 37(4): 436-445. Citado na pág. 1, 7 
Murthy et al. (2004) DN Prabhakar Murthy, Min Xie e Renyan Jiang. Weibull models, volume 505. John Wiley \& Sons. Citado na pág. 33

Nadarajah (2005) Saralees Nadarajah. Exponentiated pareto distributions. Statistics, 39(3): 255-260. Citado na pág. 1

Nadarajah (2006) Saralees Nadarajah. The exponentiated gumbel distribution with climate application. Environmetrics, 17(1):13-23. Citado na pág. 1

Nadarajah (2008) Saralees Nadarajah. On the distribution of kumaraswamy. Journal of Hydrology, 348(3):568-569. Citado na pág. 10

Nadarajah (2011) Saralees Nadarajah. The exponentiated exponential distribution: a survey, 2011. Citado na pág. 1

Nadarajah e Gupta (2007) Saralees Nadarajah e Arjun K Gupta. The exponentiated gamma distribution with application to drought data. Calcutta Statistical Association Bulletin, 59(1-2): 29-54. Citado na pág. 7

Nadarajah e Kotz (2004) Saralees Nadarajah e Samuel Kotz. The beta gumbel distribution. Mathematical Problems in Engineering, 2004(4):323-332. Citado na pág. 1, 2

Nadarajah e Kotz (2006) Saralees Nadarajah e Samuel Kotz. The beta exponential distribution. Reliability engineering $\mathcal{E} 3$ system safety, 91(6):689-697. Citado na pág. 1, 2

Ojo e Olapade (2003) Mathew Oladejo Ojo e AK Olapade. On the generalized logistic and log-logistic distributions. Kragujevac Journal of Mathematics, 25(25):65-73. Citado na pág. 1

Ortega et al. (2011) Edwin MM Ortega, Gauss M Cordeiro e Elizabeth M Hashimoto. A log-linear regression model for the beta-weibull distribution. Communications in Statistics-simulation and Computation, 40(8):1206-1235. Citado na pág. 39

Paranaíba (2012) Patrícia Ferreira Paranaíba. Caracterização e extensões da distribuição Burr XII: propriedades e aplicações. Tese de Doutorado, Universidade de São Paulo. Citado na pág. 2

Pescim et al. (2010) Rodrigo R Pescim, Clarice GB Demétrio, Gauss M Cordeiro, Edwin MM Ortega e Mariana R Urbano. The beta generalized half-normal distribution. Computational statistics $\mathscr{G}$ data analysis, 54(4):945-957. Citado na pág. 1

Santana (2010) Tiago Viana Flor de Santana. As distribuições Kumaraswamy-log-logística e Kumaraswamy-logística. Tese de Doutorado, Universidade de São Paulo. Citado na pág. 2

Silva et al. (2010) Giovana Oliveira Silva, Edwin MM Ortega e Vicente G Cancho. Log-weibull extended regression model: estimation, sensitivity and residual analysis. Statistical Methodology, 7(6):614-631. Citado na pág. 2

SOUZA et al. (2016) Glaucia Tadu de SOUZA et al. Nova generalização para a classe beta-g de distribuições de probabilidade. Citado na pág. 1

Steinbrecher (2002) G Steinbrecher. Taylor expansion for inverse error function around origin. Relatório técnico, University of Craiova working paper. Citado na pág. 12

Tahir e Cordeiro (2016) Muhammad H Tahir e Gauss M Cordeiro. Compounding of distributions: a survey and new generalized classes. Journal of Statistical Distributions and Applications, 3(1): 13. Citado na pág. 1

Tahir e Nadarajah (2015) Muhammad H Tahir e Saralees Nadarajah. Parameter induction in continuous univariate distributions: Well-established g families. Anais da Academia Brasileira de Ciências, 87(2):539-568. Citado na pág. 1 
Therneau et al. (1990) Terry $M$ Therneau, Patricia M Grambsch e Thomas R Fleming. Martingale-based residuals for survival models. Biometrika, 77(1):147-160. Citado na pág. 43 